Review

\title{
From Bush Medicine to Modern Phytopharmaceutical: A Bibliographic Review of Devil's Claw (Harpagophytum spp.)
}

\author{
Thomas Brendler 1,2 \\ 1 Department of Botany and Plant Biotechnology, University of Johannesburg, Auckland Park, \\ Johannesburg 2006, South Africa; txb@plantaphile.eu \\ 2 Plantaphile, Collingswood, NJ 08108, USA
}

Citation: Brendler, T. From Bush

Medicine to Modern

Phytopharmaceutical: A

Bibliographic Review of Devil's Claw (Harpagophytum spp.). Pharmaceuticals 2021, 14, 726. https://doi.org/ $10.3390 /$ ph14080726

Academic Editors: Thomas Efferth and Mona Abdel-Tawab

Received: 22 June 2021

Accepted: 8 July 2021

Published: 27 July 2021

Publisher's Note: MDPI stays neutral with regard to jurisdictional claims in published maps and institutional affiliations.

Copyright: (C) 2021 by the author. Licensee MDPI, Basel, Switzerland. This article is an open access article distributed under the terms and conditions of the Creative Commons Attribution (CC BY) license (https:// creativecommons.org/licenses/by/ $4.0 /)$.

\begin{abstract}
Devil's claw (Harpagophytum spp., Pedaliaceae) is one of the best-documented phytomedicines. Its mode of action is largely elucidated, and its efficacy and excellent safety profile have been demonstrated in a long list of clinical investigations. The author conducted a bibliographic review which not only included peer-reviewed papers published in scientific journals but also a vast amount of grey literature, such as theses and reports initiated by governmental as well as non-governmental organizations, thus allowing for a more holistic presentation of the available evidence. Close to 700 sources published over the course of two centuries were identified, confirmed, and cataloged. The purpose of the review is three-fold: to trace the historical milestones in devil's claw becoming a modern herbal medicine, to point out gaps in the seemingly all-encompassing body of research, and to provide the reader with a reliable and comprehensive bibliography. The review covers aspects of ethnobotany, taxonomy, history of product development and commercialization, chemistry, pharmacology, toxicology, as well as clinical efficacy and safety. It is concluded that three areas stand out in need of further investigation. The taxonomical assessment of the genus is outdated and lacking. A revision is needed to account for intra- and inter-specific, geographical, and chemo-taxonomical variation, including variation in composition. Further research is needed to conclusively elucidate the active compound(s). Confounded by early substitution, intermixture, and blending, it has yet to be demonstrated beyond a reasonable doubt that both (or all) Harpagophytum spp. are equally (and interchangeably) safe and efficacious in clinical practice.
\end{abstract}

Keywords: Harpagophytum; devil's claw; teufelskralle; grapple plant; sengaparile; harpagoside; nomenclature; ethnobotany; traditional use; trade; biochemistry; pharmacology; clinical; safety; toxicology; veterinary; review

\section{Introduction}

Devil's claw is the collective name of plants from the genus Harpagophytum (Pedaliaceae). The latter includes two species, H. procumbens (Burch.) DC. ex Meisn. and H. zeyheri Decne., currently divided into five subspecies with introgression reported from overlapping habitats $[1,2]$. The secondary root tubers of devil's claw are used in botanical drugs and supplements and are exported from Southern Africa, mainly Namibia. Entrepreneurial spirit, colonialism, and the absence of regulatory barriers drove the commercialization of devil's claw in a fashion similar to that of other medicinal plants from Southern Africa, such as Umckaloabo (Pelargonium sidoides) [3], rooibos (Aspalathus linearis) and honeybush (Cyclopia spp.) [4], buchu (Agathosma betulina) [5], cape aloe (Aloe ferox) [6], uzara (Xysmalobium undulatum) [7], and to some extent, hoodia (Hoodia gordonii) [8], among others [9]. From the 1960s onward, products quickly gained popularity, initially in Germany, then France, and by the mid-1980s, all over the developed world. This led to an increase in demand and consequently harvesting pressure in the countries of origin, to the point that devil's claw was briefly considered to be listed on CITES appendix II [10]. However, ongoing efforts to introduce good harvesting practices and cultivation attempts helped supply to become more sustainable. 
Once harvested, botanical differentiation between species and subspecies is virtually impossible, and it can safely be assumed that since the 1970s, the product of commerce is one or the other and often of mixed origin [11-14]. Thus, current official compendia do not distinguish between the two botanical sources of devil's claw but require compliance in terms of contents of the marker compound harpagoside, a cinnamoylated iridoid glucoside. The primary medicinal uses of devil's claw are the management of arthritis, pain, and dyspepsia [15,16]. An impressive number of clinical trials, the earlier being mostly observational, the more recent randomized, placebo-controlled studies-albeit being of variable quality-indicate clinical efficacy and safety [17]. However, whether harpagoside is more than a just marker, but also the (only) active compound, remains to be demonstrated. Consequently, superiority of $H$. procumbens over $H$. zeyheri cannot be derived merely from harpagoside content [18]. Lower levels of harpagoside do not necessarily translate to lower levels of total iridoids, and phytochemically distinct extracts from $H$. procumbens and $H$. zeyheri have shown similar in vivo analgesic and anti-inflammatory properties [19].

The vast body of evidence presented here-over a period of 55 years, about one general review per year was published in the scientific literature [20-80], not counting reviews specific to clinical efficacy (see Section 12.1.) -makes devil's claw one of the bestresearched botanicals. Figure 1 illustrates the growing and sustained research interest. The 694 included publications were grouped by language, which yielded a perspective on how research interest spread geographically over time. Despite English becoming the lingua franca of science toward the end of the 20th century, a trend is clearly noticeablefrom Germany to France to the rest of the world-and confirmed by research, trade, and availability and popularity of pharmaceutical products. An interesting discrepancy reveals itself when comparing the total with the research output of the region of origin. Nonetheless, knowledge gaps concerning species interchangeability remain to be closed, the elucidation of which is one purpose of this review. It is hoped that the assembly of this extensive bibliography will stimulate further research of this interesting genus of medicinal plants.

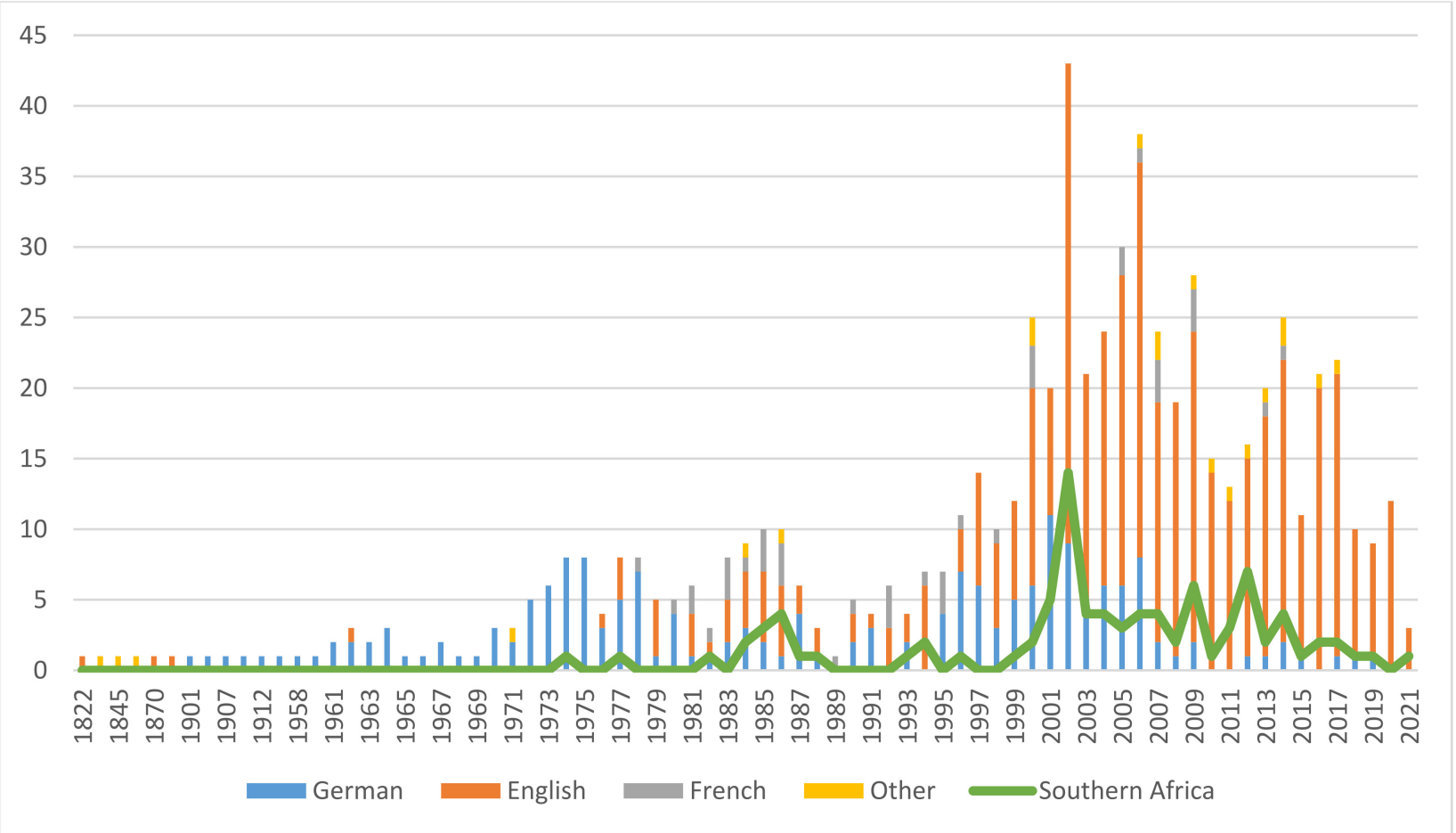

Figure 1. Publications on Harpagophytum spp., 1822-2021 (colors indicate publication language/origin of research). 


\section{Materials and Methods}

Multiple searches were conducted in the PubMed, Scopus, and Google Scholar databases with the following keywords and combinations thereof: "Harpagophytum, harpagophyton, devil(')s claw, Teufelskralle, grapple plant, sengaparile, garra-do-diabo, griffe du diable, (h)arpagoside, taxonomy, nomenclature, ethnobotany, traditional use, ecology, cultivation, sustainability, economy, trade, CITES, chemistry, biochemistry, compounds, pre-clinical, pharmacology, clinical, RCT, safety, toxicology, veterinary, review". Union catalogues were also searched. The search was limited to scientific literature, and popular magazines and compendia were excluded. Also excluded were articles which only mentioned Harpagophytum without elaboration. Further excluded were reports on compounds present in Harpagophytum, that were derived from other sources (e.g., harpagoside from Scrophularia spp.).

Reference sections of selected publications were searched manually. Academic theses were retrieved primarily via the Bielefeld Academic Search Engine (BASE). Patents were retrieved from the European, US, and international (WIPO) patent office databases.

A substantial body of publications (125) was identified addressing aspects of ecology, stakeholders' livelihoods, efforts in capacity building, as well as access-benefit-sharing (ABS) and its legislation. They are included in the publication statistics (see Figure 1). In reviewing the pharmaceutical history of devil's claw, however, these topics appear out of scope and will be reviewed in a separate publication. Figure 2 illustrates the selection process.
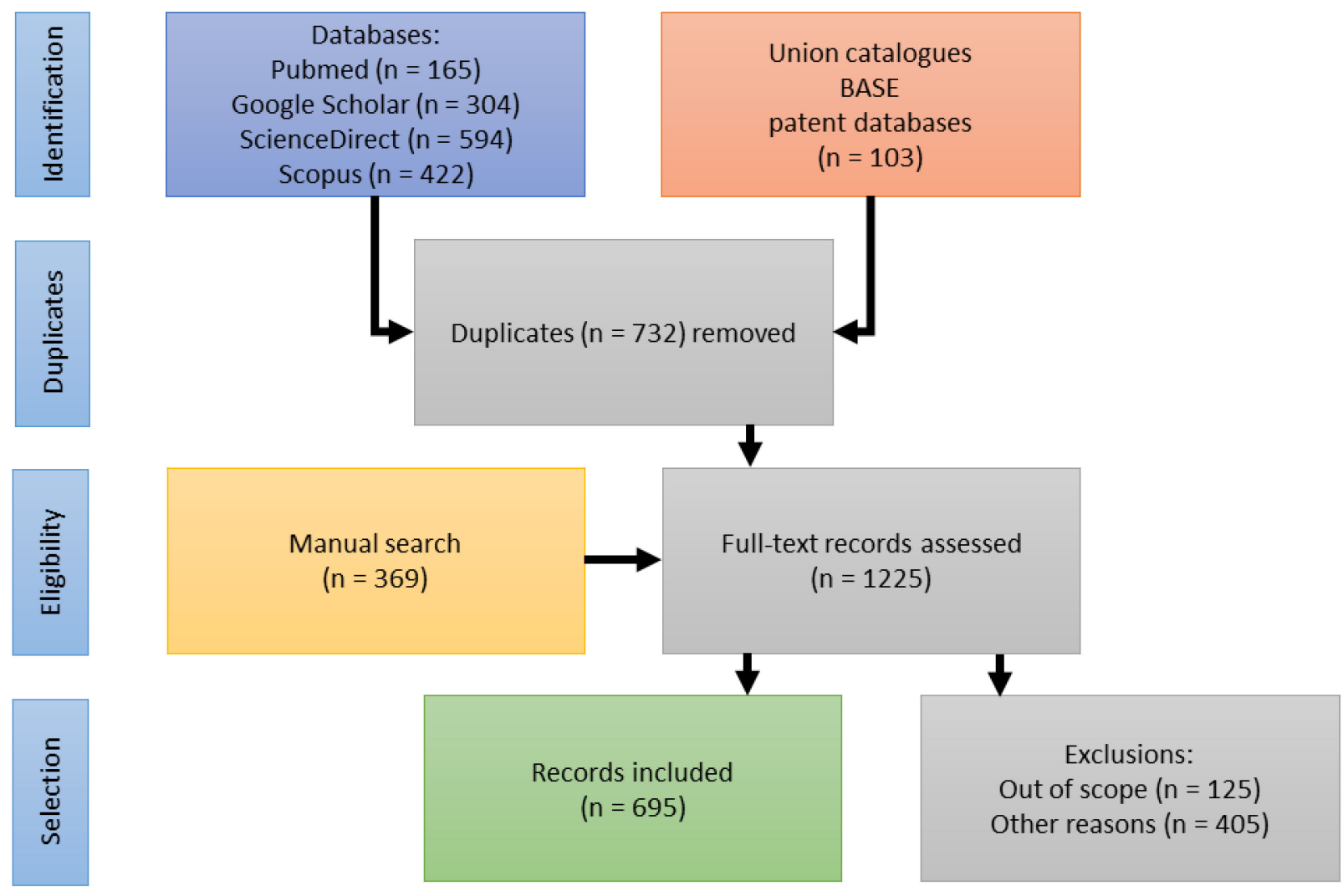

Figure 2. Flow diagram of the reference identification, screening and inclusion.

\section{Nomenclature}

\subsection{Taxonomy}

The genus Harpagophytum was first described as Uncaria Burch. by Burchell in his Travels in the interior of southern Africa (1822) [81]. However, he was apparently unaware that Uncaria had already been used by Schreber for a genus in the Rubiaceae in 1789 . 
Purportedly, de Candolle first noted this oversight, leading Meisner to describe the species as Harpagophytum procumbens DC [82]. However, de Candolle's section of the Prodomus was only published in 1845 [83], making Meisner the author of the genus and creating the following complete citation as:

Harpagophytum DC. ex Meisner, PI. Vas. Gen. 1: 298 and 2: 206 (1840), syn.: Uncaria Burch., Trav. Int. S. Afr. 1:536 (1822), nom. illegit., non Schreb. 1789; type specimen: Harpagophytum procumbens (Burch.) DC. ex Meisner, PI. Vas. Gen. 2:206 (1840); basionym: Uncaria procumbens Burch., Trav. Int. S. Afr. 1: 536 (1822).

Decaisne, in his review of the Pedalineae, attributed four distinct species to the genus: H. procumbens DC., H. burchellii Decne. (= H. procumbens), and for the first time, $H$. zeyheri and H. leptocarpum [Uncaria leptocarpa (Decne.) Ihlenf. \& Straka] [84]. The genus was last reviewed by Ihlenfeldt and Hartmann (1970) [1], who differentiated two species and five subspecies primarily based on the shape of the fruit correlated with the number of seeds. They also provide the most recent botanical descriptions for all subspecies.

Harpagophytum procumbens (Burch.) DC. ex Meisn.:

- H. procumbens (Burch.) DC. ex Meisn. ssp. procumbens-(1).

- H. procumbens (Burch.) DC. ex Meisn. ssp. transvaalense Ihlenf. \& H. Hartm.-(2). Harpagophytum zeyheri Decne.:

- H. zeyheri Decne. ssp. zeyheri-(3).

- H. zeyheri Decne. ssp. schijffii Ihlenf. \& H. Hartm.-(4).

- H. zeyheri Decne. ssp. sublobatum (Engler) Ihlenf. \& H. Hartm.-(5).

The numbers in parentheses represent the respective species in Figure 3 below.

Synonymy:

- H. burchellii Decne. $=$ H. procumbens ssp. procumbens DC. ex Meisn.

- H. zeyheri f. sublobatum Engl. = H. zeyheri ssp. sublobatum (Engl.) Ihlenf. \& H. Hartm.

- H. procumbens var. sublobatum (Engl.) Stapf = H. zeyheri ssp. sublobatum (Engl.) Ihlenf. \& H. Hartm.

- H. peglerae Stapf = H. zeyheri ssp. zeyheri Decne.

Interspecific introgression has been described [85] and shown to be reflected in morphometric measurements, and DNA profiles. Both species and all their putative hybrids also showed geographical variation in biochemical composition $[2,85-90]$.

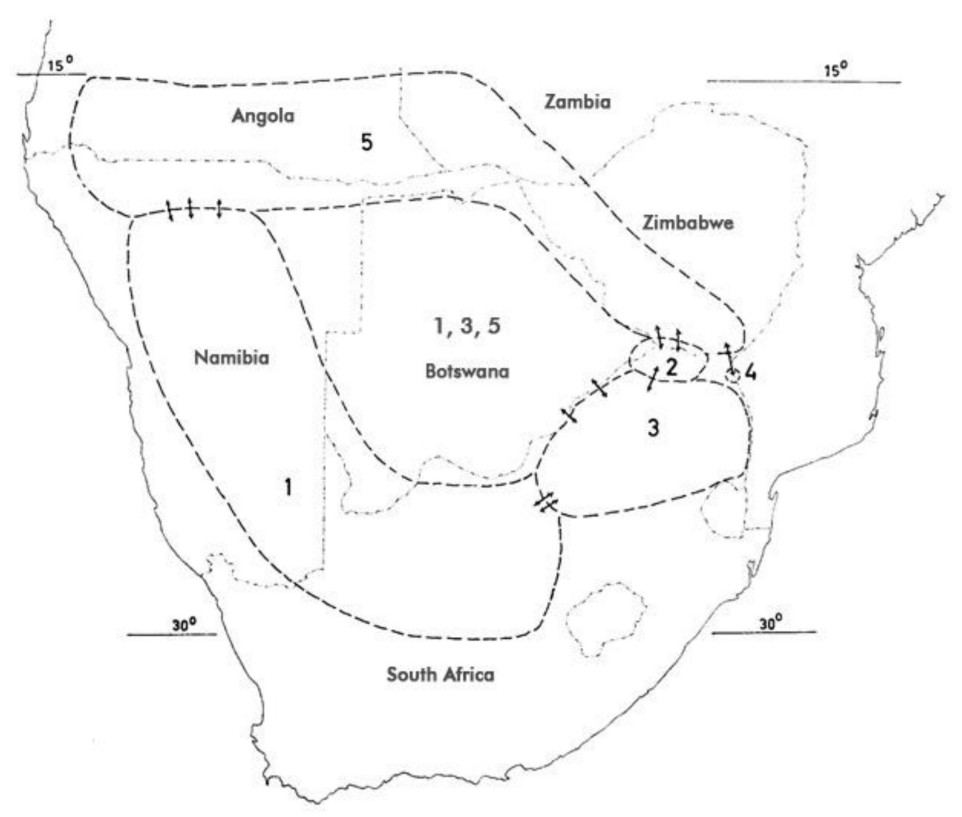

Figure 3. Distribution of H. procumbens and H. zeyheri (after $[1,64,91])$. For numerical attribution of species, see Section 3.1. Arrows indicate introgression. 


\subsection{Vernacular Names}

Teufelskralle, Trampelkette (Ger.); devil's claw, grapple plant (Eng.); garra-do-diabo (Port.); garra del diablo (Esp.); artiglio del diavolo (It.); griffe du diable (Fr.); sengaparile (Tswana), duiwelsklou, kloudoring, duiwelsdoring, sanddoring, beesdubbeltjie, wolspinnekop (Afr.); otjihangatene (Oshiherero);/ / khuripe / / khams, gamagu (Nama/Damara); elyata, omalyata (Oshikwanyama); ekatata, makakata (Oshindonga/Kwangali); likakata (Gciriku/Shambyu); !ao!ao,// xsamsa-//oro,/ / xemta f'eisa (Kung); I l am-si- I I q'oa-ka (West !Xoon), malamatwa (Silozi) [92-94].

\section{Distribution}

In the context of species interchangeability in commerce, it is noteworthy that the long-time assumption that only $H$. procumbens occurs in Namibia was disproved as early as the late 19th century. Ihlenfeldt discussed collections from the Etosha pan and later from the Kaokoveld and the Caprivi strip holding specimen of H. zeyheri [95]. Baum (1903) reported H. procumbens (Burch.) DC. var. sublobatum Engl. [= H. zeyheri Decne. ssp. sublobatum (Engler) Ihlenf. \& H. Hartm.] from near lake Camelungo in southern Angola [96]. Cultivation has been experimented with in northern South Africa and, more recently, in Namibia, however, it has thus far neither proven very successful nor commercially viable [97-99].

\section{Ethnobotany}

Interestingly, there are no records for indigenous use of devil's claw until the beginning of the 20th century. Two accounts from the 19th century by Wood [100] and Cooke [101] (Figure 4) were the only ones that could be found making reference to devil's claw (as grapple plant-Uncaria procumbens) but focus on its "devilish nature": "The reader may easily imagine the horrors of a bush which is beset with such weapons. No one who wears clothes has a chance of escape from them. If only one hooked thorn catches but his coat-sleeve, be is a prisoner at once. [ ... I If the reader would like to form an idea of the power of these thorns, he can do so by thrusting his arm into the middle of a thick rose-bush, and mentally multiplying the number of thorns by a hundred, and their size by fifty" [100].
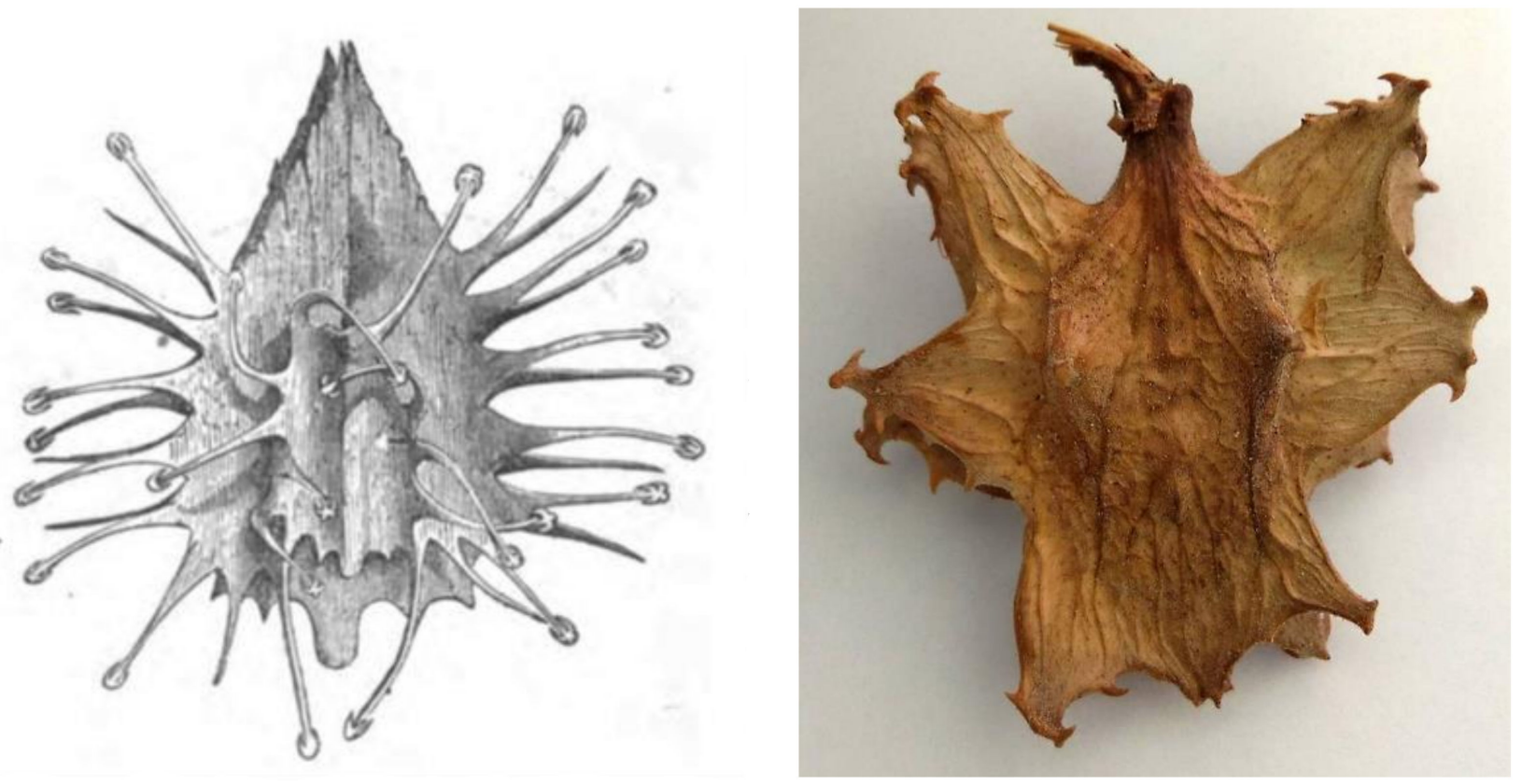

Figure 4. Fruit of the "Grapnel" (note the misspelling!) plant from [101] vs. an actual fruit (photograph by the author).

Lübbert, in 1901, provided the first unambiguous account for the use of "KuriKhamiknollen" (= tubers of $/ /$ khuripe $/ /$ khams $=$ Harpagophytum $)$ in wound healing [102] 
In 1907, Hellwig, medical officer of the imperial protection forces in German South-West Africa (Namibia), compiled a report on medicinal plant uses of the indigenous population, including an account of the Herero Samuel Kariko of the use of "otjihangatene" (=Harpagophytum) to treat cough, diarrhea, constipation, and venereal diseases [103]. Dinter, in 1909 and 1912 [104,105], utilized this report for his account of local food plants, but unfortunately omitted to include medicinal uses because he considered them unverified [106]. The fact that Hellwig provided an explicit source renders the colorful story of how the use of devil's claw was "discovered" by Mehnert implausible and more likely part of a marketing strategy (see below) [107].

Later accounts corroborated these early records of traditional use of devil's claw tubers primarily in the form of infusions and decoctions for digestive purposes, midwifery, pain relief, fever, diabetes, as a general tonic, for infectious diseases, and the dry powder topically as a wound dressing [40,92,108-112]. Ethnoveterinary uses in poultry have also been reported from Botswana [113]. It must be noted, however, that none of the early records clearly differentiate between species. It can only be speculated based on the origin of the records that Nama/Herero may have referred to H. procumbens, whereas reports from Botswana would concern mostly $H$. zeyheri.

\section{Economy}

\subsection{History of Commercialization}

The story around how a soldier of the Kaiserliche Schutztruppe (German "imperial protection forces") and later a farmer in Mariental (Namibia) Gottreich Hubertus Mehnert came across devil's claw is firmly anchored in the scientific literature. Sometime during the so-called Hottentot uprising from 1904 to 1908 (in fact, a brutal war and genocide of the German troops primarily against the Herero and Nama tribes, which has most recently been recognized by the German government [114]), after observing a local healer successfully improving the condition of a gravely wounded local, he questioned the healer about the magic remedy, but the healer refused to disclose the place from where he had collected it. Purportedly, access to one of the most successful botanical drugs of modern times can be attributed to Mehnert's pointer dog [107].

This version, however, must be relegated to the world of "romance" and seen as part of an elaborate marketing campaign-it is repeated in many slightly altered versions by multiple authors. Mehnert doubtlessly experimented with the root and found it effective in a variety of ailments, but the discovery of its medicinal powers ought to be attributed first and foremost to the native tribes and secondarily to Lübbert and Hellwig (see above) with whom they shared their knowledge. It was sheer luck that nobody else developed an interest, allowing Mehnert to consolidate his "research" and to commence commercialization. He eventually shared it, while being interned at camp Andalusia during the 2nd World War, with another "collateral" prisoner, German scientist O. H. Volk, who had visited German South-West Africa at the wrong time [115]. In the camp's botanical society, knowledge was freely exchanged, which allowed Volk to return home to Germany with likely an entire laundry list of interesting plants. The introduction of devil's claw (and probably also rooibos) to Germany can be attributed to him [58]. He shared his knowledge with Zorn who conducted some initial pharmacological research [116] and then initiated himself a flurry of investigations elucidating devil's claw's basic chemistry [117-128]. Meanwhile, in the early 1950s, Mehnert trademarked "Harpago" and started exporting to Germany. Erwin Hagen trademarked "Harpago" in Germany in the early 1960s and began to market it as an infusion and later in homoeopathic preparations [129] (Figure 5). "Harpagosan" tea was registered as a botanical drug in Germany in 1977 [130]. 


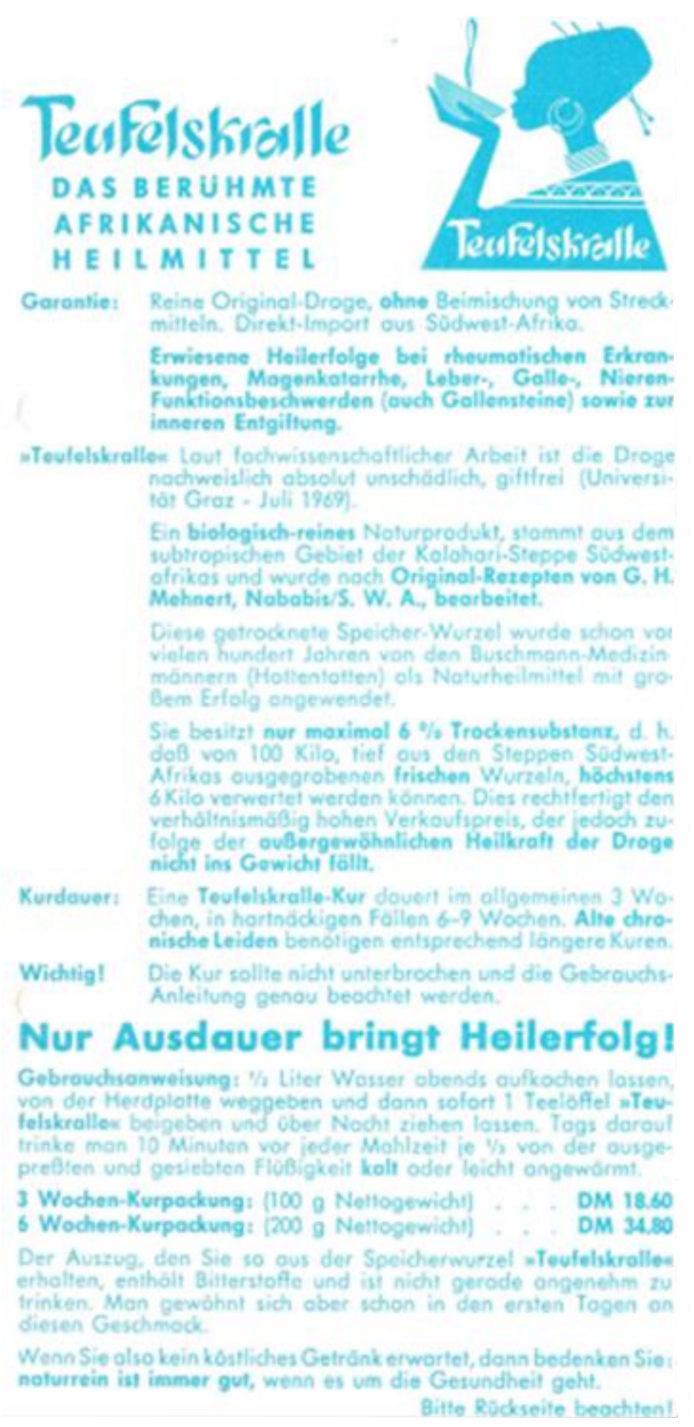

Devil's claw, the famous African medicine

Guarantee: pure, original drug, undiluted, imported directly from South-West Africa.

Success proven for rheumatic diseases, dyspepsia, livergallbladder and kidney dysfunction, internal detoxification.

According to scientific investigation (University of Graz, July 1969), the drug is absolutely harmless and nontoxic.

A pure product of nature from the sub-tropical region of the South-West African Kalahari, processed according to the original recipe from G. H. Mehnert, Nababis/S.W.A.

This dried tuber has been used medicinally by the bushman healers (hottentots) for hundreds of years with great success.

The dry weight of the tuber is only $6 \%$, e.g., $100 \mathrm{~kg}$ freshly excavated tubers from the steppes of South-West Africa, make up only $6 \mathrm{~kg}$ dried drug. This justifies the relatively high retail price, which, given the drug's amazing healing powers, remains negligible in comparison.

A treatment cycle is 3 weeks on average but can be up to 6-9 weeks in severe cases. Chronic ailments require longer treatments. Important: the treatment should not be interrupted, and instructions must be followed.

Only endurance brings success!

Instructions for use: bring $0.5 \mathrm{~L}$ of water to a boil in the evening, remove from heat and add 1 tsp devil's claw, let steep overnight. Strain and drink $1 / 3$ of the liquid 3 times daily, 10 min before meals, cold or tepid.

3-week treatment (100 g) DM 18.60 (US\$ $4.75 *$ ).

6-week treatment (200 g) DM 34.80 (US\$ $8.88^{*}$ ).

The devil's claw root decoction contains bitter principles and is not exactly palatable, but in a few days, one gets used to the flavor. So, do not expect a delicious drink and consider that natural is always good for your health.

* Adjusted for inflation, this equates to approximately US\$ 35 and US\$ 65, in today's money, respectively.

Figure 5. Advertisement Fa. Hagen (early 1970s).

What follows is a story of extensive biochemical, pharmacological, toxicological, and clinical investigation, and the development of multiple standardized pharmaceuticals, initially in Germany (the German drug information system AMIce alone lists a total of 434 products, most of which, however, are no longer active, see, e.g., [131]), and since the 1980s, also in France and elsewhere [132]. Demand quickly started to grow exponentially, and concerns were raised over the sustainability of harvesting practices [133-135]. In response to unsustainable harvesting and poor processing practices, the Namibian Devil's Claw Exporter's Association Trust became part of a Good Agricultural and Collection Practice (GACP) project in which it intends to ensure that Namibian devil's claw is sustainably harvested and processed according to GACP guidelines.

\subsection{Trade}

Market demands impact livelihoods and policymakers alike. Trends indicate the health of an industry and inform resource assessments as well as regulatory interventions. With the following breakdown of trade and export data, I intend to address a controversy around species interchangeability, namely how the ingredient is regulated in the finished product markets. Hagen and others created a demand which local suppliers struggled to meet [133-135]. Sustainable collection and harvesting practices and governmental oversight were largely absent until $\sim 1975$. When originally only $H$. procumbens had been collected, driven by the economic boom, the collection and admixture of $H$. zeyheri commenced as 
early as the 1970s [11-14]. Furthermore, albeit on a much smaller scale than Namibia, both South Africa and Botswana [136-138] began to participate in the export market, also adding $H$. zeyheri into the supply chain (for distribution see above). Nott [14] and Taylor and Moss [138] broke down data specific to importing countries and explicitly listed importers, respectively. It is therefore safe to state that all importing markets have received either both species or mixtures thereof as early as the late 1970s. European regulators acknowledged the commercial reality by adding $H$. zeyheri to pharmacopeial monographs (see Section 6), while the US, for instance, remained oblivious to this practice, which stirred a controversy over the regulatory compliance and legitimacy of products containing $H$. zeyheri in 2015 [139]. The following overview of export volumes (Figure 6) is compiled from multiple sources $[10,14,18,136-138,140-151]$ and further informed by the Namibian Ministry of Environment and Tourism (MET). The MET stopped sharing its data-based on export permits-with the public in 2015. According to one of the most prominent Namibian exporters of devil's claw, the years 2015-2020 saw a slight increase in demand, peaking in 2019 at around 1000 metric tons, otherwise averaging around 700 metric tons annually. Materials in trade (both species) fall into four categories: conventional (lowest) quality makes up about $80 \%$ of the trade volume, GACP quality currently contributes about $10-15 \%$ to the total, though efforts are underway to dramatically increase this proportion, certified organic quality adds organic certification to GACP-compliant material and makes up about $5-10 \%$ of the total trade volume, and finally, organic and Fair for Life certified material (H. procumbens only) contributes $\sim 1 \%$ to the trade total. Prices per $\mathrm{kg}$ (for full container loads, cost and freight) range from $€ 4.00$ (H. zeyheri) and $€ 6.70$ (H. procumbens) for conventional quality, via $€ 5.40$ (H. zeyheri) and $€ 8.20$ (H. procumbens) for GACP quality, and $€ 7.20$ (H. zeyheri) and $€ 8.50$ (H. procumbens) for organic quality, to $€ 9.00$ for Fair for Life certified material (pers. comm. G. Diekmann, EcoSo Dynamics cc, Namibia). While these prices and volumes make this a sizeable industry, it must be noted that most of the value is of course added during the manufacture of pharmaceuticals in the target markets. It is also noteworthy that over all this time, Namibian exports may have been bolstered by (illegal) imports from Angola and Zambia, for which—naturally—no records exist [152].

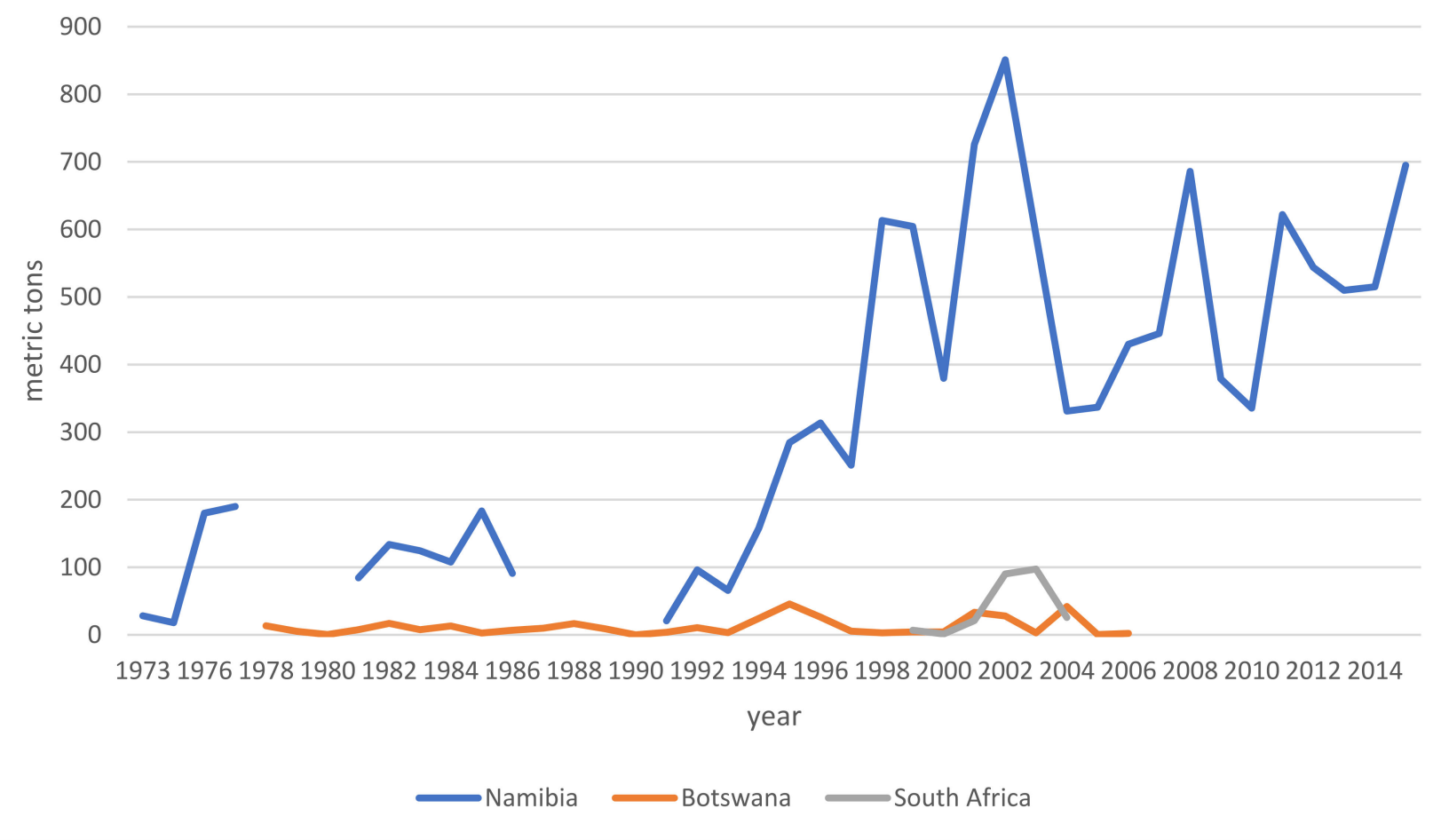

Figure 6. Devil's claw exports by country—gaps reflect years in which no data was reported. 


\section{Representation in Pharmacopeias and Authoritative Compendia}

Given its presence in the European marketplace since the 1950s and in the US at least since the late 1970s, pharmacopeial standards for devil's claw were set surprisingly late, likely due to suitable analytical methods not being available. While a qualitative assessment for the bitterness value according to the German Pharmacopoeia 7 (DAB 7) was suggested as early as 1977 [11], no specific monograph for devil's claw was included in DAB until 1993, which, in fact, required testing for harpagoside content (see Table 1 below). The first monograph in Europe appeared in the British Herbal Pharmacopoeia in 1981. Devil's claw first appeared in the European Pharmacopoeia in 1995, H. zeyheri, however, was not included as an allowable source species until 2003. The US Pharmacopeia, on the other hand, does not have a monograph for devil's claw other than a draft proposal published in the Herbal Medicines Compendium in 2013 [153].

Table 1. Representation of devil's claw in pharmacopeias and authoritative compendia.

\begin{tabular}{|c|c|c|c|}
\hline Source & Species Included & Year & Reference \\
\hline \multicolumn{4}{|c|}{ Official monographs } \\
\hline British Herbal Pharmacopoeia & H. procumbens & 1981 & [154] \\
\hline Pharmacopée française & H. procumbens & 1989 & [155] \\
\hline Kommission E & $\begin{array}{l}\text { H. procumbens (corrected) } \\
\text { Monograph was informed by }[65,156]\end{array}$ & 1990 & [157] \\
\hline Pharmacopée française & H. procumbens dry extract & 1992 & [158] \\
\hline DAB 10 2nd. Supplement & H. procumbens & 1993 & [159] \\
\hline European Pharmacopoeia 3rd ed. & H. procumbens & 1997 & [160] \\
\hline $\begin{array}{l}\text { European Pharmacopoeia 4th ed. } \\
\text { Suppl. } 4.3\end{array}$ & H. procumbens/H. zeyheri (revised) & 2003 & [161] \\
\hline Pharmacopée française & $\begin{array}{l}\text { H. procumbens/H. zeyheri } \\
\text { (homoeopathic preparations) }\end{array}$ & 2007 & [162] \\
\hline European Pharmacopoeia 7th ed. & Devil's claw dry extract & 2008 & [163] \\
\hline Health Canada & H. procumbens & 2008 & [164] \\
\hline European Pharmacopoeia 7th ed. & H. procumbens/H. zeyheri (revised) & 2011 & [165] \\
\hline Polish Pharmacopoeia 8 & H. procumbens/H. zeyheri & 2008 & [166] \\
\hline USP Herbal Medicines Compendium & H. procumbens/H. zeyheri (draft) & 2013 & [153] \\
\hline European Medicines Agency (EMA) & H. procumbens/H. zeyheri (revised from 2008) & 2016 & {$[15,167,168]$} \\
\hline European Pharmacopoeia 9.6 & H. procumbens/H. zeyheri (revised) & 2018 & [169] \\
\hline State Pharmacopoeia of Ukraine & H. procumbens/H. zeyheri & 2018 & [170] \\
\hline Health Canada & H. procumbens/H. zeyheri (revised from 2008) & 2018 & [164] \\
\hline \multicolumn{4}{|c|}{ Authoritative compendia } \\
\hline ESCOP & H. procumbens & 1996 & [171] \\
\hline ESCOP & $\begin{array}{l}\text { H. procumbens (revised) } \\
\text { (omission of H. zeyheri is discussed in }[172,173] \text { ) }\end{array}$ & 2003 & [16] \\
\hline World Health Organization & H. procumbens & 2007 & [174] \\
\hline African Herbal Pharmacopoeia & H. procumbens & 2010 & [175] \\
\hline Martindale & H. procumbens (continuously revised from 1997) & 2017 & [176] \\
\hline \multicolumn{4}{|c|}{ Other compendia } \\
\hline Longwood Herbal Task Force & H. procumbens/H. zeyheri & 1999 & [177] \\
\hline Herbal Medicines & H. procumbens/H. zeyheri & 2015 & [178] \\
\hline Phytopharmacy & H. procumbens/H. zeyheri & 2015 & [179] \\
\hline Kooperation Phytopharmaka & H. procumbens & 2020 & [180] \\
\hline
\end{tabular}




\section{Biochemistry}

After Volk's return to Germany (see Section 6.1) and following Zorn's first pharmacological study of devil's claw in 1958 [116], the university of Würzburg (Germany) became a research hotspot for the elucidation of active and suitable marker compounds in devil's claw for decades to come. The effort was largely concluded by the end of the 1980s and comparatively little has been added to this effort since. Table 2 lists all publications focused on the biochemical composition. For analytical methods and quality control, see Section 9 .

Iridoid-glycosides, primarily harpagoside, harpagide, and procumbide; phytosterols; phenylpropanoids such as verbascoside; triterpenes, such as oleanolic acid, $3 \beta$-acetyloleanolic acid, and ursolic acid; flavonoids, such as kaempferol and luteolin; unsaturated fatty acids, cinnamomic acid, chlorogenic acid, and stachyose were identified as the most prominent compounds present in the root. Figure 7 shows the chemical structures of the primary iridoid glucosides present in Harpagophytum root.

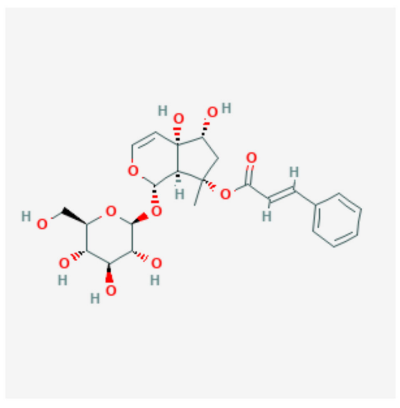

harpagoside

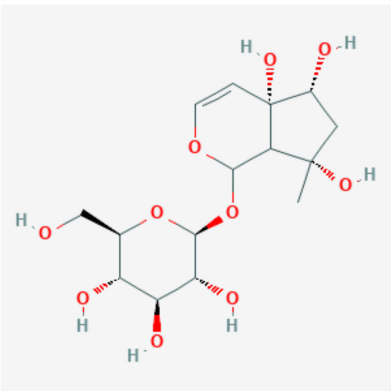

harpagide

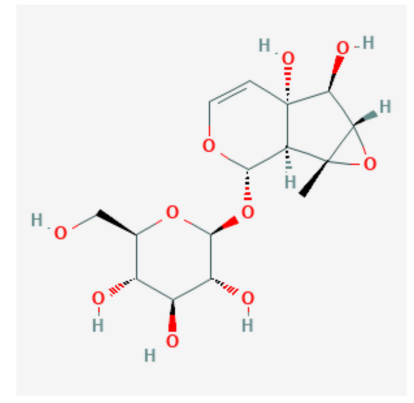

procumbide

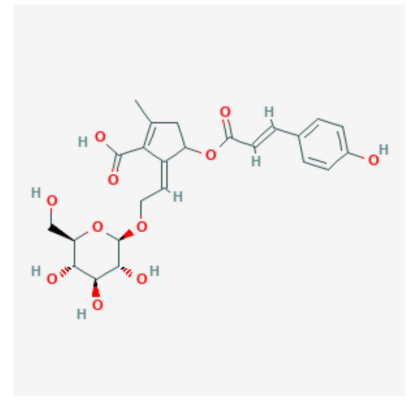

pagoside

Figure 7. Iridoid glucosides present in devil's claw root (source PubChem).

Interestingly, the biosynthetic pathway for harpagoside is not yet well-elucidated. The first step resulting in geranyl diphosphate is still considered to be under debate [17], since while the principal steps are known, some intermediates remain hypothetical and dependent on the "chosen" pathway. Georgiev and colleagues [30] propose two different routes to the formation of geranyl diphosphate from the condensation of dimethylallyl diphosphate and isopentenyl diphosphate, the latter being supplied through either the mevalonate or the mevalonate-independent pathways. Geraniol is synthesized by geraniol diphosphate synthase and hydroxylated to form 8-hydroxygeraniol, followed by two oxidation steps and isomerization into 8-epi-iridodial. Carboxylation and glycosylation form its glycoside, which, in turn, is transformed into harpagide through decarboxylation and oxidations. Finally, harpagoside emerges as the product of cinnamoyl esterification at the 3-hydroxyl position.

Several studies have investigated differences in the quantitative composition of different Harpagophytum species, subspecies, and hybrids [19,181-184], and found the composition to be highly variable, depending on the material used, collection location, natural variation within the taxa, environmental influences, processing, and analytical methods. Content of the marker compound harpagoside is generally lower in H. zeyheri and has been found to be between $0 \%, 1 \%$, and $4 \%$ in $H$. procumbens and between $0 \%$ and $3 \%$ in H. zeyheri. Verbascoside and isoverbascoside contents in $H$. procumbens varied between $0.2 \%$ and $0.4 \%$ and $0.2 \%$ and $1 \%$, respectively. Pagoside content in $H$. procumbens varied between $0.06 \%$ and $0.16 \%$. Hybrids showed the highest contents for most key compounds except harpagoside. 8-p-Coumaroylharpagide content in $H$. zeyheri varies between $0.7 \%$ and $1.4 \%$, while being effectively absent in $H$. procumbens. The lower harpagoside content in $H$. zeyheri has in the past driven controversies over species equivalence in terms of clinical efficacy, however, this debate seems futile as a marker compound is not necessarily the (only) active one. Indeed, the pre-clinical research (outlined in Section 10) indicates that activities of multiple rather than single compounds may contribute to the overall effect. 
Table 2. Elucidation of the biochemical composition of devil's claw root.

\begin{tabular}{|c|c|c|}
\hline Topic & Year & Reference \\
\hline Isolation and characterization of harpagoside & 1960 & [117] \\
\hline Stachyose, raffinose, and a further glucoside in the aqueous phase & 1961 & [118] \\
\hline Characterization of harpagoside & 1961 & [119] \\
\hline Isolation and characterization of harpagoside and harpagide & 1962 & [120] \\
\hline Characterization of harpagoside & 1962 & [121] \\
\hline Characterization of harpagide & 1963 & [122] \\
\hline Isolation of stachyose and a further glucoside & 1963 & [123] \\
\hline Characterization of harpagoside & 1964 & [124] \\
\hline Isolation of procumbide & 1964 & [125] \\
\hline Structural characterization of harpagoside & 1966 & [126] \\
\hline Characterization of procumbide and further constituents & 1967 & [127] \\
\hline Characterization of procumbide & 1968 & [128] \\
\hline Characterization of a chinone and other constituents & 1970 & [185] \\
\hline Characterization of procumbide & 1971 & [186] \\
\hline Further constituents & 1974 & [187] \\
\hline Elucidation of triterpene esters & 1975 & [188] \\
\hline Overview of known mono-, di-, and sesquiterpenoids with pharmacological activity & 1977 & [189] \\
\hline Elucidation of a resin, an essential oil, and a mucilaginous fraction & 1978 & [190] \\
\hline Structural characterization of procumbide & 1979 & [191] \\
\hline Glucose, galactose, fructose, myo-inositol, sucrose, raffinose, and stachyose identified & 1979 & [192] \\
\hline Preparation and structure of harpagogenine & 1981 & [193] \\
\hline Carbohydrates and harpagoside in tissue cultures and roots of devil's claw & 1982 & [194] \\
\hline New iridoids: 8-O-(p-coumaryl)-harpagide and procumboside & 1983 & [195] \\
\hline Novel iridoid and phenolic compounds & 1987 & [196] \\
\hline Three pyridine monoterpene alkaloids from harpagoside and commercial extract & 1999 & [197] \\
\hline Review of iridoids & 2000 & [198] \\
\hline Review of composition (both species) & 2002 & [199] \\
\hline Two diterpenes, $(+)-8,11,13$-totaratriene-12,13-diol and ferruginol & 2002 & [200] \\
\hline New iridoid- and phenylethanoid glycosides & 2003 & [201] \\
\hline Acetylated phenolic glycosides & 2003 & [202] \\
\hline Pharmacological characterization of harpagoside & 2004 & [203] \\
\hline Chinane-type tricyclic diterpenes and other minor compounds & 2006 & [204,205] \\
\hline Review of iridoids and other compounds & 2006 & {$[206,207]$} \\
\hline Review of chemical constituents & 2007 & [208] \\
\hline Elucidation and characterization of compounds with specific pharmacologic profiles & 2008 & {$[209,210]$} \\
\hline New triterpenoid glycoside, harproside, and new iridoid glycoside, pagide & 2010 & [211] \\
\hline Kynurenic acid content & 2013 & [212] \\
\hline New iridoid diglucoside & 2016 & [213] \\
\hline
\end{tabular}




\section{Analytical Methods and Quality Control}

The quickly increasing popularity of devil's claw products required an ongoing effort to develop and refine tools to identify and quantify devil's claw in its raw, processed, and finished product states. Initially, the primary aims were identification and contaminants [214,215], later, standardization [11] and quality control [216,217], and finally identification and quantification methods to support pharmacological and clinical research. Early methods, however, did not account for species differentiation, i.e., simple pharmacy-proof methods of the 1970s would likely not have been able to differentiate between $H$. procumbens and $H$. zeyheri. In fact, methods and equipment refined enough to do so, regardless of the extent of processing, only became available in the 1990s. Analysis of retention samples retrospectively determined the presence of both species in commercial products. Table 3 provides a quick reference to publications of methods of quality control in chronological order. In current practice, the most commonly used methods for identification and assaying devil's claw raw materials and products include TLC, HPLC, HTPLC, and LC/MS, for instance, the current edition of the European Pharmacopoeia employs microscopy and TLC for identification and LC for harpagoside quantification; more recently, chemometric modeling and hyperspectral imaging have emerged as promising methods for species differentiation.

Table 3. Analytical methods and methods of quality control.

\begin{tabular}{|c|c|c|}
\hline Topic & Year & Reference \\
\hline Macroscopic and microscopic descriptions & 1964 & [58] \\
\hline Macroscopic, microscopic, and chromatographic differentiation of commercial drug samples & 1973 & [218] \\
\hline Macroscopic, microscopic, and chromatographic differentiation of commercial drug samples & 1974 & [219] \\
\hline Simple TLC with Scrophularia nodosa as a reference standard & 1975 & [220] \\
\hline Distribution of harpagoside within $H$. procumbens and $H$. zeyheri & 1977 & [221] \\
\hline Standardization by determination of harpagoside, bitterness value, and dry residue & 1977 & [11] \\
\hline Spectrometric method for the quantitative evaluation of the glycoiridoids & 1978 & [222] \\
\hline Report of falsified, adulterated, and contaminated commercial products & 1978 & [214] \\
\hline Quantitative determination of harpagoside via HPLC & 1980 & [223] \\
\hline GLC method for the determination of harpagide and harpagoside & 1981 & [224] \\
\hline Histological characteristics under scanning electron microscope & 1984 & [225] \\
\hline Stability of iridoids during extraction & 1985 & [226] \\
\hline Determination of harpagoside, luteolin, chlorogenic, caffeic, and cinnamic acid from extracts & 1986 & [227] \\
\hline Analysis of permethylated iridoid glycosides by GC/MS & 1986 & [228] \\
\hline Determination of harpagide, 8-p-coumaroyl harpagide (8-PCHG), and harpagoside by HPLC & 1994 & [229] \\
\hline Analysis of the harpagoside content of commercial samples by HPLC & 1995 & [230] \\
\hline TLC method for determination of harpagoside & 1995 & [231] \\
\hline HPLC/UV for the determination of harpagoside in commercial powdered dry extracts & 1996 & [232] \\
\hline HPLC/UV for the determination of harpagoside in commercial tea products & 1996 & [233] \\
\hline HPLC/UV for the determination of harpagoside in commercial products (multiple dosage forms) & 1996 & [234] \\
\hline HPTLC for quantitative determination of harpagoside & 1996 & [235] \\
\hline $\begin{array}{l}\text { HPLC determination of harpagide, 8-PCHG, and harpagoside in } H \text {. procumbens and } H \text {. } \\
\text { zeyheri-ratio of harpagoside/8-PCHG can be used to distinguish species }\end{array}$ & 1997 & [19] \\
\hline HPLC determination of ratio of harpagoside/8-PCHG, 8-PCHG $<8 \%$ proposed for H. procumbens & 1998 & [12] \\
\hline Methods for quality control and stability testing of Harpagophytum homeopathic preparations & 1998 & [236] \\
\hline HPLC/UV for the determination of harpagoside in commercial dry extract products & 1999 & [237] \\
\hline
\end{tabular}


Table 3. Cont.

\begin{tabular}{|c|c|c|}
\hline Topic & Year & Reference \\
\hline $\begin{array}{l}\text { Differentiation of H. procumbens (<9\% 8-PCHG), mixtures (10-30\% 8-PCHG), and H. zeyheri } \\
\text { (>31 8-PCHG) proposed }\end{array}$ & 2000 & [13] \\
\hline Biopharmaceutical quality, release of active ingredients in vitro, and disintegration tests & 2000 & [238] \\
\hline Methods for detection of adulterations and contaminations & 2001 & [239] \\
\hline Bioequivalence of Harpagophytum products & 2002 & [240] \\
\hline Near infrared spectroscopy (NIRS) determination of harpagoside, 8-PCHG, and their ratio & 2003 & [241] \\
\hline NIR-FT-Raman spectroscopy for identification and quantification of harpagoside & 2005 & [242] \\
\hline Determination of harpagoside from $\mathrm{CO}_{2}$-extracts with HPLC and HPTLC-densitometry & 2005 & [243] \\
\hline NIRS determination of harpagoside, $8-\mathrm{PCHG}$, and their ratio & 2005 & [244] \\
\hline Fast HPLC determination of harpagoside using a monolithic silica column & 2005 & [245] \\
\hline Validation of a fast-HPLC for separation of iridoid glycosides to distinguish between species & 2005 & [246] \\
\hline LC-DAD-MS/SPE-NMR hyphenation for identification of isobaric iridoid glycoside regioisomers & 2005 & [247] \\
\hline X-ray fluorescence spectrometry (SRTXRF) to determine trace elements & 2005 & [248] \\
\hline Determination of aflatoxin $B_{1}$ & 2006 & [249] \\
\hline LC/MS determination of harpagoside, 8-PCHG, and their ratio & 2006 & [250] \\
\hline Computational study to estimate the proton and sodium cation affinities of harpagide & 2006 & [251] \\
\hline Quality parameters of finished products in the German market & 2006 & [252] \\
\hline Proposal to revise the drug-extract ratio of aqueous/ethanolic extracts & 2006 & [253] \\
\hline Methods for determination of minerals and heavy metals & 2007 & [254] \\
\hline Analysis of iridoids in horse urine & 2008 & [255] \\
\hline $\begin{array}{l}\text { Solid-phase extraction for LC/MS analysis of harpagoside, 8-PCHG, and harpagide in } \\
\text { equine plasma }\end{array}$ & 2008 & [256] \\
\hline Validated HPTLC method for the determination of harpagoside & 2008 & [257] \\
\hline $\begin{array}{l}\text { High-Pressure Liquid Chromatography-Diode Array Detection (HPLC-DAD) for harpagoside and } \\
\text { isoacteoside contents }\end{array}$ & 2009 & [258] \\
\hline HPLC-DAD and HPLC-ESI-MS analyses of stability of the constituents & 2011 & [181] \\
\hline Anatomical study of secondary tubers and quantification of harpagoside by HPLC & 2012 & [259] \\
\hline Authenticity and contamination tests by DNA barcoding & 2013 & [260] \\
\hline Exploring species substitution through chemometric modeling of ${ }^{1} \mathrm{H}-\mathrm{NMR}$ and UHPLC-MS & 2014 & [182] \\
\hline $\begin{array}{l}\text { Mid-infrared spectroscopy and short-wave infrared hyperspectral imaging for qualitative } \\
\text { assessment of H. procumbens and H. zeyheri }\end{array}$ & 2014 & {$[85,88]$} \\
\hline Morphology, histochemistry, and ultrastructure of foliar mucilage-producing trichomes & 2014 & [261] \\
\hline NMR-based chemometric approach for species differentiation & 2014 & [262] \\
\hline UPLC Q-TOF ESI determination of harpagosides in H. procumbens, H. zeyheri, and extracts & 2016 & [263] \\
\hline Loss on drying and total ash & 2016 & [264] \\
\hline $\begin{array}{l}\text { Comparison of microwave and ultrasound-assisted with conventional solvent extraction methods } \\
\text { for harpagoside determination }\end{array}$ & 2016 & [265] \\
\hline $\begin{array}{l}\text { Innovative micro-extraction techniques to determine harpagoside and phenolic patterns in } \mathrm{H} \text {. } \\
\text { procumbens and finished products }\end{array}$ & 2017 & [266] \\
\hline Determination of suitable extraction solvent & 2017 & [267] \\
\hline GC-MS determination of chemical constituents & 2017 & [268] \\
\hline DNA barcoding to detect contamination and substitution & 2017 & [269] \\
\hline HPLC and MS analyses of spagyric tinctures & 2019 & [270] \\
\hline
\end{tabular}


Table 3. Cont.

\begin{tabular}{lccc}
\hline \multicolumn{1}{c}{ Topic } & Year & Reference \\
\hline $\begin{array}{l}\text { Validated RP-HPLC-PDA method for quantification of harpagoside in extracts and } \\
\text { finished products }\end{array}$ & 2019 & [271] \\
\hline UPLC-MS profiling of samples from different locations & 2019 & {$[90]$} \\
\hline Determination of macro- and micro-elements in finished products using ICP OES & 2020 & {$[272]$} \\
\hline HPLC method for harpagoside determination in finished product (tablet) & 2020 & {$[273]$} \\
\hline
\end{tabular}

\section{Processing, Products, Applications}

The majority of data on processing and delivery systems is provided in the list of patents compiled in Section 14. EMA's HMPC assessment report on H. procumbens and/or H. zeyheri, radix, provides an overview of extracts that are most commonly used in commercial products [167]:

- $\quad$ Liquid extract $(1: 1 ; 30 \%$ v/v ethanol)

- $\quad$ Soft extract (2.5-4.0:1; 70\% v/v ethanol)

- $\quad$ Dry extract (1.5-2.5:1; water)

- Dry extract (5-10:1; water)

- Dry extract $(2.6-4: 1 ; 30 \%$ v/v ethanol)

- $\quad$ Dry extract $(1.5-2.1: 1 ; 40 \%$ v/v ethanol)

- $\quad$ Dry extract (3-5:1;60\% v/v ethanol)

- $\quad$ Dry extract $(3-6: 1 ; 80 \%$ v/v ethanol)

- $\quad$ Dry extract $(6-12: 1 ; 90 \%$ v/v ethanol)

- $\quad$ Tincture (1:5), extraction solvent ethanol $25 \%(v / v)$

Figure 8 shows the processing from harvest to the raw material in commerce. Historically, teas $[67,274]$, e.g., Harpagosan (see above), fluidextracts [42,67], spray-dried aqueous extract $[26,67]$, homeopathic preparations for both oral (p.o.) and intraperitoneal (i.p.) application $[26,27,67]$, and powder in capsules $[26,67,93]$ were also common galenic forms. The European Pharmacopoeia stipulates a minimum of $1.2 \%$ of harpagoside in the raw material [169]. Dry extracts were standardized to contain a minimum of $1.5 \% \mathrm{~m} / \mathrm{m}$ of harpagoside [167].

More recently, Plaizier-Vercammen and Bruwier evaluated the impact of excipients on friability and hygroscopicity of direct compression of a spray-dried Harpagophytum extract [275]. Günther et al. analyzed the parameters affecting supercritical fluid extraction with $\mathrm{CO}_{2}$ of harpagoside [276]. Performance of a topical preparation with devil's claw extract on acrylic acid polymers base compared to ketoprofen was assessed by PiechotaUrbanska and colleagues [277]. Both formulations demonstrated rheological stability and high pharmaceutical availability. Almajdoub described a freeze-dried aqueous extract of $H$. procumbens encapsulated in lipid vesicles by using a dry film hydration technique with and without further alginate coating for optimal (delayed) release and small intestine absorption [278]. Development of a gastro-resistant coated tablet prepared from a standardized hydroethanolic root extract for the purpose of more effective delivery and consequent dose reduction was reported by Lopes et al. [279]. 

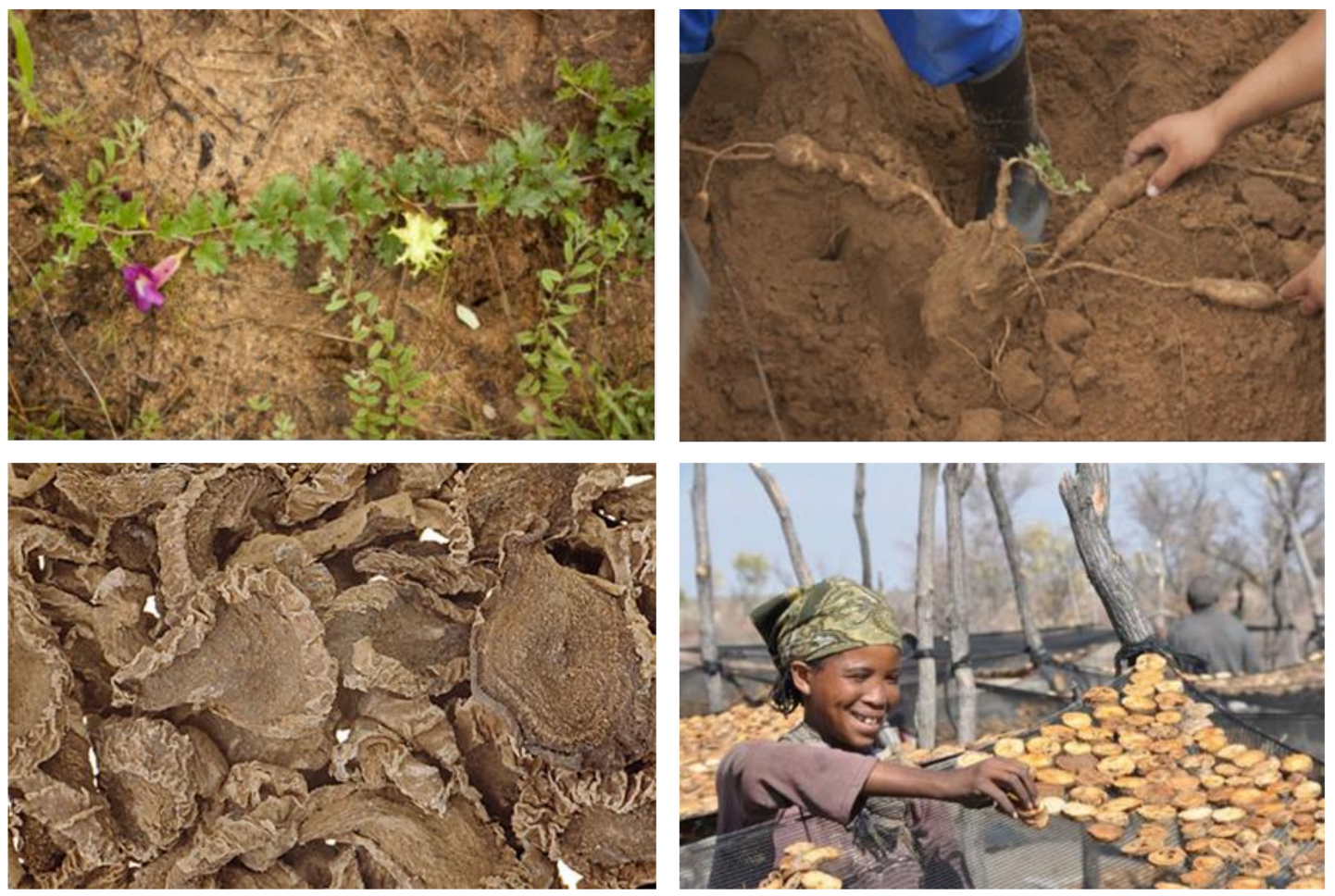

Figure 8. Clockwise: H. procumbens, secondary tubers, drying of the sliced tubers, article of commerce (photographs by the author). The article of commerce shown here is conventional quality (see Section 6). Note the difference in color of the slices shown on the bottom right, which were harvested and processed in compliance with GACP.

\section{Pre-Clinical Research}

\subsection{Pharmacology}

Studies mainly investigated anti-inflammatory activities and were conducted with various extracts, extract fractions, or isolated compounds. Harpagophytum iridoid compounds are considered the primary actives, to which anti-inflammatory, antinociceptive, analgesic, antimicrobial, chemopreventive, hepatoprotective, neuroprotective, and immunomodulatory effects are commonly attributed $[189,198,209,280,281]$. As cyclooxygenase (COX)-1/2 inhibitors have emerged as important targets for treating rheumatoid arthritis, the influence on the arachidonic acid pathway has been a research focus. The most commonly used methods for measuring peripheral analgesic activity were the various forms of the writhing tests, hot-plate test, and the Randall-Selitto test in rats and mice. To demonstrate anti-inflammatory effects, different animal models of inflammation were commonly used, e.g., the carrageenan-induced mouse/rat paw edema, the 12-O-tetradecanoylphorbol-13acetate (TPA)-induced mouse edema, the granuloma pouch test, zymosan-induced arthritis, albumin-induced rat paw edema, adjuvant-induced arthritis in rats ( $M$. tuberculosis; Freund adjuvant), and Adriamycin-induced rat paw edema. More advanced in vivo and a variety of in vitro and ex vivo models were developed and employed over time (see Tables $4-7$ below). 
Table 4. In Vitro experiments regarding analgesic/antinociceptive and anti-inflammatory effects of devil's claw preparations and compounds.

\begin{tabular}{lcc}
\hline \multicolumn{1}{c}{ Study } & Year & Reference \\
\hline $\begin{array}{l}\text { Guineapig isolated ileum; harpagoside }(40 \mu \mathrm{g} / \mathrm{mL}) \text { and harpagogenine } \\
(2.5 \mu \mathrm{g} / \mathrm{mL}) \text { non-selectively inhibited contractions; harpagide }(40 \mu \mathrm{g} / \mathrm{mL})\end{array}$ & 1981 & Fontaine et al. [282] \\
increased the cholinergic response without inhibitory effects. & & Benito et al. [283] \\
\hline $\begin{array}{l}\text { Calcium ionophore-stimulated mouse peritoneal macrophages; harpagoside } \\
\text { and harpagide inhibited leukotriene C4 (LTC4) and prostaglandin E2 (PGE2) } \\
\text { release (not significant) and harpagoside inhibited thromboxane B2 (TXB2) } \\
\text { release, similar to ibuprofen. }\end{array}$ & 2000 & Fiebich et al. [284] \\
$\begin{array}{l}\text { Lipopolysaccharide-stimulated primary human monocytes; Harpagophytum * } \\
\text { extract, harpagoside, and harpagide extract prevented synthesis of tumor } \\
\text { necrosis factor alpha (TNF- } \alpha) \text {, isolated substances showed no effect. }\end{array}$ & 2001 & Loew et al. [285] \\
\hline $\begin{array}{l}\text { Ionophore A23187 stimulated Cys-LT levels in anticoagulated whole blood; } \\
\text { Harpagophytum extract, harpagoside, and extract fractions; inhibitory effect } \\
\text { stronger with extract than harpagoside, no effect with fractions without } \\
\text { harpagoside, suggesting relation between serum harpagoside and inhibition of } \\
\text { leukotriene biosynthesis. }\end{array}$ & 2001 & \\
\hline $\begin{array}{l}\text { Modified Hens-Egg-Test at the Chorion-Allantoin-Membrane (HET-CAM) and } \\
\text { lipoxygenase assay; ethanolic extracts of Harpagophytum (60\%, 30\%, 0\%); 30\% } \\
\text { most potent in HET-CAM, 60\% most potent in inhibiting lipoxygenase } \\
\text { pathway. }\end{array}$ & 2002 & Wahrendorf et al. [286] \\
\hline
\end{tabular}

Human neutrophile elastase (HNE); Harpagophytum extract, fractions, and isolates; weak dose-dependent inhibition was observed, with H. procumbens extract twice as strong as $\mathrm{H}$. zeyheri; $6^{\prime}$-O-acetyl-acteoside (not in $\mathrm{H}$. (dominant in H. zeyheri).

Lipopolysaccharide (LPS)-induced inflammation in mouse fibroblast cell line L929; 3-(4,5-dimethylthiazol-2-yl)-2,5-diphenyltetrazolium bromide (MTT) assay, reverse transcription-polymerase chain reaction, PGE2 immunoassay, and nitric oxide (NO) detection; aqueous $H$. procumbens extract; suppression of

PGE2 synthesis and NO production.

Human chondrocytes stimulated with interleukin (IL)-1 $\beta$; Harpagophytum dry extract $(210,480 \mathrm{mg})$; immunofluorescence and Western blot analyses showed dose-dependent suppression of matrix metalloproteinases production via inhibition of cytokine expression.

Bovine and human chondrocytes, stimulated with LPS and IL-1 $\beta$, respectively; Harpagophytum extracts $(100,33,1 \mu \mathrm{g} / \mathrm{mL})$; significant suppression of PGE2 expression and NO synthase in human chondrocytes (bovine experiment was flawed).

2006 Chrubasik [289], Chrubasik et al. [290], Hadzhiyski et al. [291]

Human whole-blood assay, human polymorph nuclear leucocytes (PMNL) assay; COX-2, 5-lipogenase (LOX) inhibition, respectively; comparison of Harpagophytum aqueous-ethanolic and $\mathrm{CO}_{2}$ extracts $(2 \%, 20 \%$, and $30 \%$ harpagoside, respectively); variable but weak PGE2 inhibition for all, superiority of $\mathrm{CO}_{2}$ extracts in 5-LOX inhibition.

Human HepG2 hepatocarcinoma and RAW 264.7 macrophage cell lines; harpagoside (0.1-200 $\mu \mathrm{M})$; LPS-induced mRNA, COX-2 expression, and inducible nitric oxide (iNOS) inhibited, and NF- $\mathrm{BB}$ activation suppressed.

LPS-stimulated THP-1 cells; incubated with $50 \mu \mathrm{g} / \mathrm{mL}$ H. procumbens dry extract (DER 1.5-3); microarray (gene chip) assay; noted inhibition of several inflammatory targets. 
Table 4. Cont.

\begin{tabular}{lcc}
\hline \multicolumn{1}{c}{ Study } & Year & Reference \\
\hline $\begin{array}{l}\text { Isolated murine macrophages; H. procumbens crude methanolic extract, } \\
\text { harpagoside, phenylethanoid-containing fraction, verbascoside; strong } \\
\text { inhibitory action related to NO and TNF- } \alpha \text { and IL-6 production, and COX-1 } \\
\text { and COX-2 expression, comparable to harpagoside. }\end{array}$ & 2011 & Gyurkovska et al. [296]
\end{tabular}

LPS-stimulated human monocytes and mouse RAW264.7 macrophages; molecular targets; $H$. procumbens ethanolic extract (2.9\% harpagoside); dose-dependent inhibition of TNF- $\alpha$, IL-6, IL-1 $\beta$, PGE2, and COX-2, inhibition of activator protein (AP)-1 pathway without affecting NF- $k B$ and mitogen-activated protein (MAP) kinase pathways.

Pre-transdermal and post-transdermal COX-2 inhibition and permeation studies; H. procumbens extract, harpagoside, harpagide; hydroxypropyl cellulose gels (carrier) with permeation enhancers tested on synthetic membranes, with and without enhancers on human skin, Azone ${ }^{\circledR}$ enhancer Ebrahim [298] chosen, direct COX-2 inhibition maintained (pre-permeation $80 \%$, post-permeation $77 \%$ COX-2)

LPS-stimulated monocytic THP-1 cells; enzyme-linked immunosorbent assays, WST-1 assay; Harpagophytum extract; dose-dependent suppression of TNF- $\alpha$, IL-6, IL-8, independent from external metabolic activation.

Primary human osteoarthritis chondrocytes; harpagoside $(600 \mu \mathrm{M})$; significant reduction in IL-1 $\beta$-induced expression of IL-6, no effect on nuclear levels of NF-кB.

Haseeb et al. [300,301]

Differentiated 3T3-L1 adipocytes; harpagoside; activation of peroxisome proliferator-activated receptor (PPAR)- $\gamma$, significant inhibition of TNF- $\alpha$-induced mRNA synthesis and production of atherogenic adipokines including IL-6, plasminogen activator inhibitor-1, and monocyte chemoattractant protein-1.

IFN- $\gamma$ /LPS-stimulated THP-1 cells; harpagoside and harpagide; decreased TNF- $\alpha$-secretion in PMA-differentiated THP-1 cells, positive effect on TNF- $\alpha$ and intercellular adhesion molecule-1 mRNA-expression in undifferentiated cells.

Human synovial membranes from subjects with and without osteoarthritis; $H$. procumbens extract, multiple solvents; cannabinoid type 2 (CB2) receptor enhanced, phosphatidylinositol-specific phospholipase $C \beta 2$ downregulated with water and DMSO, fatty acid amide hydrolase (FAAH) activity inhibited with all.

* Species not specified; however, all specific attribution must be cautioned against due to the frequent admixture.

Table 5. In Vivo experiments regarding analgesic/antinociceptive and anti-inflammatory effects of devil's claw preparations and compounds.

\begin{tabular}{lcc}
\hline \multicolumn{1}{c}{ Study } & Year & Reference \\
\hline $\begin{array}{l}\text { Formaldehyde-induced arthritis in rats; Harpagophytum * infusion p.o. and } \\
\text { subcutaneous; significant reduction of swelling, subcutaneous application } \\
\text { better tolerated. }\end{array}$ & 1958 & Zorn [116] \\
\hline $\begin{array}{l}\text { Albumin-induced paw edema, granuloma-pouch-test, formaldehyde-induced } \\
\text { arthritis in rats, rabbit ear-withdrawal test; whole extract and harpagoside, } \\
\text { intravenous (i.v.) and i.p.; some (significant) effects shown similar to those of } \\
\text { phenylbutazone. }\end{array}$ & 1970 & Eichler and Koch [305] \\
\hline $\begin{array}{l}\text { Rats; blood panel; Harpagophytum aqueous extract 3:1, 30 mg/kg; triglycerides, } \\
\text { uric acid, urea, and cholesterol significantly reduced. }\end{array}$ & 1974 & Int. Bio Research [306] \\
\hline $\begin{array}{l}\text { Dextran-induced paw edema; rats; Harpagophytum, aqueous extract 3:1; edema } \\
\text { significantly reduced. }\end{array}$ & 1974 & Int. Bio Research [307] \\
\hline
\end{tabular}


Table 5. Cont.

\begin{tabular}{lcc}
\hline \multicolumn{1}{c}{ Study } & Year & Reference \\
\hline $\begin{array}{l}\text { Eight Harpagophytum dry extracts, p.o. and i.v., tested for analgesic and } \\
\text { antiphlogistic effects in five animal models; some analgesic and antiphlogistic } \\
\text { effects with methanolic, butanolic, and fluid extracts; pure harpagoside }\end{array}$ & 1978 & Erdös et al. [308] \\
superior, semi-chronic models showed better results. & & \\
\hline
\end{tabular}

Carrageenan-induced rat paw edema (30) and adjuvant-induced arthritis in rats (40); Harpagophytum 100-1000 mg/kg, single dose and 21 days; no significant effect in the edema model, some effect in the arthritis model at the McLeod et al. [309] higher dose.

Carrageenan-induced rat paw edema; aqueous ethanolic crude extract of Harpagophytum and various fractions; only crude extract effective, concludes that harpagoside is likely not the (only) active.

1986 Duband [274]

Carrageenan-induced rat paw edema; methanolic extract of Harpagophytum; dose-dependent edema inhibition.

1990

Mánez et al. [310]

Carrageenan-induced rat paw edema; aqueous extract of Harpagophytum (1.8\% harpagoside) and harpagoside i.p.; significant reduction of edema with extract, not with harpagoside.

Adriamycin-induced rat paw edema; Harpagophytum, 37, 370, and $3700 \mathrm{mg} / \mathrm{kg}$; dose-dependent edema inhibition up to $48 \%$ after one hour; compared to control (Adriamycin only) effect transient after 5 days.

1992

Jadot and Lecomte [312]

Carrageenan-induced mouse paw edema and TPA-induced mouse ear edema; harpagoside (p.o. and topically); no notable protective effects.

Del Carmen Recio et al. [313]

Carrageenan-induced rat paw edema; aqueous extracts of Harpagophytum (400 and $800 \mathrm{mg} / \mathrm{kg}, 2.72 \%$ harpagoside) i.p. pre-treatment, p.o., and intraduodenally; significant inhibition i.p. and intraduodenally, no effect orally.

Soulimani et al. [314]

Carrageenan-induced mouse paw edema; Harpagophytum and Uncaria tomentosa extracts; no effect on inflammatory response individually, but significant effect combined.

Freund's adjuvant-induced arthritis in rats; acute $(25,50$, or $100 \mathrm{mg} / \mathrm{kg})$ or chronic $(100 \mathrm{mg} / \mathrm{kg})$ treatments with $\mathrm{H}$. procumbens solution; increased 'latency of paws' withdrawal and reduction in paw edema, compared to control.

Fresh egg albumin-induced pedal edema in rats, hot-plate and acetic acid tests in mice; H. procumbens root aqueous extract $(50-800 \mathrm{mg} / \mathrm{kg}$ i.p.); significant effect against nociceptive pain stimuli and significant, dose-dependent reduction of edema.

Abe et al. [315]

Carrageenan-induced back-paw edema, Freund's adjuvant-induced arthritis, cotton pellet-induced granuloma, and writhing tests in rats and mice; Harpagophytum aqueous extract ( $800 \mathrm{mg} / \mathrm{kg}$ bw), acetyl salicylic acid and indomethacin as controls; significant effects in all models similar to indomethacin and acetyl salicylic acid.

TPA-induced COX-2 expression in mouse skin; Harpagophytum methanolic extract $(200,400 \mu \mathrm{g})$ topically prior to TPA application; significant inhibition of COX-2 expression, COX-1 unchanged, no effect on NF- $\mathrm{KB}$.

Carrageenan-induced back-paw edema in rats; H. procumbens extract $(100,200$, 400 , or $800 \mathrm{mg} / \mathrm{kg}$ ) p.o. and i.p.; reduced intensity of inflammatory response when given i.p.

Adult female white New Zealand rabbits, anterior cruciate ligament transected, and medial meniscus removed; Harpagophytum extract (150 mg/day), standard food pellets as control; outcome suggests suppression of metalloproteinase-2 production.

Andersen et al. [316]

Male ICR mice; formalin test; Harpagophytum extract (1.9\% harpagoside, 30-300 mg/kg); significant dose-dependent attenuation of licking/biting and spinal nitrites/nitrates.
2004 Mahomed and Ojewole [317], Mahomed [318]

$2005 \quad$ Ahmed et al. [319]

$2005 \quad$ Kundu et al. [320]

$2006 \quad$ Catelan et al. [321]

2006 Chrubasik et al. [322], Chrubasik [289]

Uchida et al. [323] 
Table 5. Cont.

\begin{tabular}{|c|c|c|}
\hline Study & Year & Reference \\
\hline $\begin{array}{l}\text { Rabbits after unilateral meniscectomy and transection of the anterior cruciate } \\
\text { ligament; thickness, surface area, and volume of the tibial condylar cartilage } \\
\text { per MRI; H. procumbens extract ( } 14 \% \text { harpagoside); difference in thickness and } \\
\text { volume between healthy and operated leg slightly but not significantly smaller } \\
\text { with Harpagophytum. }\end{array}$ & 2011,2014 & $\begin{array}{l}\text { Wachsmuth et al. [324], Wrubel } \\
\text { [325] }\end{array}$ \\
\hline $\begin{array}{l}\text { BALB/c mice infected with Salmonella enteritidis; leukocytes, neutrophils, and } \\
\text { mononuclear cell counts, TNF- } \alpha \text {, IL- } 4,10,12 \text {, histopathological analysis of the } \\
\text { liver and small intestine; H. procumbens extract ( } 150 \mu \mathrm{g} \text { /day); downregulation } \\
\text { of cell counts, TNF- } \alpha \text {, IL-10 m } 12 \text {, IL- } 4 \text { increased, histopathology of liver } \\
\text { unchanged, hypertrophy in the small intestine, reduced with Harpagophytum. }\end{array}$ & 2014 & Bisinotto [326] \\
\hline $\begin{array}{l}\text { Male SD rats; plantar incision and spared nerve injury; mechanical withdrawal } \\
\text { threshold (MWT) test and ultrasonic vocalization (USVs); H. procumbens } \\
\text { ethanolic extract ( } 300 \mathrm{mg} / \mathrm{kg} \text {, p.o.); MWT significantly increased, USVs reduced. }\end{array}$ & 2014 & Lim et al. [327] \\
\hline $\begin{array}{l}\text { Rats; carrageenan-induced mechanical allodynia and thermal hyperalgesia, } \\
\text { involvement of the hemeoxygenase }(\mathrm{HO})-1 \text { /carbon monoxide }(\mathrm{CO}) \text { pathway; } \\
\text { H. procumbens extract ( } 300 \text { and } 800 \mathrm{mg} / \mathrm{kg} \text { i.p.); pretreatment with } \mathrm{HO} \text { inhibiter } \\
\text { reduced anti-hyperalgesic effect, pretreatment with hemin- or CO-releasing } \\
\text { molecule induced antiallodynic response. }\end{array}$ & 2015 & Parenti et al. [328] \\
\hline $\begin{array}{l}\text { Rats; formalin-induced damage to cartilage tissue; combination of } \\
\text { glucosamine hydrochloride, chondroitin sulfate, methylsulfonylmethane, } \\
\text { Harpagophytum extract ( } 3 \% \text { harpagoside), and bromelain extract }(500 \mathrm{mg} / \mathrm{kg} \text { ); } \\
\text { malondialdehyde, NO, } 8 \text {-hydroxyguanine, IL- } 1 \beta \text {, and TNF- } \alpha \text { significantly } \\
\text { lowered, glutathione significantly increased. }\end{array}$ & 2015 & Ucuncu et al. [329] \\
\hline $\begin{array}{l}\text { Rats, chronic constriction injury (CCI) of left sciatic nerve model; } \\
\text { Harpagophytum extract + morphine, each at sub-analgesic dose; significant } \\
\text { antiallodynic and anti-hyperalgesic effect suggesting synergistic effect. }\end{array}$ & 2016 & Parenti et al. [330] \\
\hline $\begin{array}{l}\text { Immunological angiogenesis induced by bronchoalveolar lavage (BAL) cells } \\
\text { grafted into BALB/c mice skin; ethanolic extract of Harpagophytum, Filipendula } \\
\text { ulmaria, and Echinacea purpurea; significant reduction of newly formed blood } \\
\text { vessels } 1.2 \text { and } 0.6 \text { mg daily. }\end{array}$ & 2016 & $\begin{array}{l}\text { Radomska-Lesniewska et al. } \\
\text { [331] }\end{array}$ \\
\hline
\end{tabular}

* Species not specified; however, all specific attribution must be cautioned against due to the frequent admixture.

Table 6. Ex vivo experiments regarding analgesic/antinociceptive and anti-inflammatory effects of devil's claw preparations and compounds.

\begin{tabular}{|c|c|c|}
\hline Study & Year & Reference \\
\hline $\begin{array}{l}\text { Human whole-blood anticoagulated with heparin; preincubated with } \\
\text { Harpagophytum * extract or purified harpagoside; both dose-dependently inhibited } \\
\text { cysteinyl-leukotriene and thromboxane B2 release after biotransformation. }\end{array}$ & 1996,1997 & Tippler et al. [332,333] \\
\hline $\begin{array}{l}\text { Human whole-blood assay (healthy and osteoarthritic) for COX-1 and COX-2 } \\
\text { activity and NO production; } H \text {. procumbens extract and harpagoside; increased the } \\
\text { activity of baseline COX-1 and COX-2 without LPS, crude extract did not alter } \\
\text { COX activity; harpagoside inhibited COX-1, COX-2, and NO. }\end{array}$ & 2007 & Anauate [334] \\
\hline $\begin{array}{l}\text { Freshly excised porcine skin; dermal and transcutaneous delivery and effect on } \\
\text { COX-2 expression in Western blotting and immunocytochemical assays; } \\
\text { Harpagophytum extract in various vehicles, harpagoside, harpagide, } \\
\text { 8-coumaroylharpagide, and verbascoside; ratio-dependent inhibition of COX-2 } \\
\text { expression, higher penetration of all compounds from ethanol/water. }\end{array}$ & 2008 & $\begin{array}{c}\text { Abdelouahab and Heard } \\
\qquad[335,336]\end{array}$ \\
\hline
\end{tabular}

Freshly excised porcine skin; transcutaneous delivery and effect on COX-2, PGE2, 5-LOX, and inducible NO synthase (iNOS) expression in Western blotting and immunocytochemical assays; commercial Harpagophytum extracts, harpagoside, harpagide, 8-coumaroylharpagide, and verbascoside; ratio-dependent inhibition of 
Table 6. Cont.

\begin{tabular}{lcc}
\hline \multicolumn{1}{c}{ Study } & Year & Reference \\
\hline $\begin{array}{l}\text { LPS-stimulated human whole-blood assay (healthy) for COX-1 and COX-2 activity } \\
\text { and NO production, incubation of isolated fractions obtained by flash } \\
\text { chromatography monitored with HPLC, TLC, and identified by }{ }_{1} \text { HNMR; fractions } \\
\text { of H. procumbens extract; highest concentration of harpagoside inhibited COX-1, }\end{array}$ & 2010 & Anaute et al. [340] \\
$\begin{array}{l}\text { COX-2, and NO; iridoid pool increased COX-2 while NO and COX-1 activities } \\
\text { remained unchanged, fraction containing cinnamic acid reduced NO only. }\end{array}$ & & \\
\hline
\end{tabular}

* Species not specified; however, all specific attribution must be cautioned against due to the frequent admixture.

Table 7. Mixed experiments regarding analgesic/antinociceptive and anti-inflammatory effects of devil's claw preparations and compounds.

\begin{tabular}{ccccc}
\hline Study & Type & Year & Reference \\
\hline
\end{tabular}

Carrageenan-induced rat paw edema and adjuvant-induced arthritis in rats; arachidonic acid and prostaglandin synthetase incubated with various concentrations of indomethacin, acetylsalicylic acid, or Harpagophytum extract (not specified); no effect on edema, anti-inflammatory activity is not mediated by the inhibition of the prostaglandin synthetase.

Cultured human mammary epithelial cells and female ICR mice; TPA-induced COX expression; Harpagophytum methanolic extract $(10,5,1 \mu \mathrm{g} / \mathrm{mL}, 600,300,60 \mu \mathrm{g}$, respectively); inhibition of COX-2 expression in both models.

\begin{tabular}{|c|c|c|c|}
\hline $\begin{array}{l}\text { Rat adjuvant-induced chronic arthritis model, LPS-stimulated } \\
\text { mouse macrophage cells (RAW 264.7); Harpagophytum ethanolic } \\
\text { extract; significant anti-inflammatory effect, and dose-dependent } \\
\text { suppression of, IL-6 and TNF- } \alpha \text {, respectively. }\end{array}$ & In Vitro and in vivo & 2010 & Inaba et al. [343] \\
\hline $\begin{array}{l}\text { Molecular docking study of harpagoside and harpagide with } \\
\text { COX-2; binding energies were }-9.13 \text { and }-5.53 \mathrm{kcal} / \mathrm{mol} \\
\text { respectively, finding both harpagoside and harpagide to be highly } \\
\text { selective COX- } 2 \text { inhibitors. }\end{array}$ & Simulation & 2016 & Rahimi et al. [344] \\
\hline $\begin{array}{l}\text { Mouse myoblast C2C12, human colorectal adenocarcinoma } \\
\text { HCT116 cell lines, isolated rat colon challenged with LPS; aqueous } \\
\text { Harpagophytum extract }(1-1000 \mu \mathrm{g} / \mathrm{mL}) ; \mathrm{HCT} 116 \text { viability reduced, } \\
\text { ROS production in both cell lines reduced, PGE2, } 8 \text {-iso-PGF } 2 \alpha, \\
\text { serotonin, and TNF- } \alpha \text { production inhibited. }\end{array}$ & In Vitro and ex vivo & 2017 & $\begin{array}{l}\text { Locatelli et al. [345], } \\
\text { Leporini et al. [346] }\end{array}$ \\
\hline $\begin{array}{l}\text { Antioxidant capacity, leukocyte ROS production, COX-2/PGE2 } \\
\text { pathway or cytokine secretions; } H \text {. procumbens methanolic extract; } \\
\text { decreased the secretion of IL-21 and IL-23, increased TNF- } \alpha, \text { IL-8, } \\
\text { and IFN- } \gamma \text {, immune-stimulant effect. }\end{array}$ & In Vitro and ex vivo & 2019 & Cholet et al. [347] \\
\hline $\begin{array}{l}\text { LPS-stimulated wild-type (C57/BL6) male mice colon and HCT116 } \\
\text { cells; experimental model of inflammatory bowel disease; } H \text {. } \\
\text { procumbens aqueous extract; anti-inflammatory, antioxidative, and } \\
\text { antimicrobial effects (against pathogen fungal strains), } \\
\text { morphological alterations in the colon tissue indicated. }\end{array}$ & In Vitro and ex vivo & 2020 & Recinella et al. [348] \\
\hline
\end{tabular}
* Species not specified; however, all specific attribution must be cautioned against due to the frequent admixture.

Investigated targets for anti-inflammatory effects and their respective $\mathrm{IC}_{50}$ (significant inhibitions, primary sources only) are summarized in Table 8. 
Table 8. Anti-inflammatory targets of Harpagophytum preparations and compounds.

\begin{tabular}{|c|c|c|c|c|c|c|c|c|c|}
\hline & & & & & $\mathrm{IC}_{50}$ & & & & Reference \\
\hline Extract/Fraction & $\underset{(\%)}{\text { Harpagoside }}$ & Cys-LT & $\mathrm{TXB}_{2}$ & $\begin{array}{l}\text { Enzyme } \\
\text { Inhibitors }\end{array}$ & IL-6 & IL-1 $\beta$ & NF- $k B$ & COX-2 & \\
\hline \multirow[t]{2}{*}{$\begin{array}{c}\text { Special extract } \\
\text { WS1531 }\end{array}$} & 7.3 & $9.2 \mu \mathrm{M} / \mathrm{L}$ & $55.3 \mu \mathrm{M} / \mathrm{L}$ & & & & & & {$[332,333]$} \\
\hline & 7.3 & $62 \mu \mathrm{g} / \mathrm{mL}$ & $373 \mu \mathrm{g} / \mathrm{mL}$ & & & & & & [285] \\
\hline \multirow[t]{4}{*}{$\begin{array}{l}\text { Aqueous } \\
\text { ethanolic } H . \\
\text { procumbens } \\
\text { extract }\end{array}$} & 2.1 & $1450 \mu \mathrm{g} / \mathrm{mL}$ & - & & & & & & [285] \\
\hline & & & & $\begin{array}{c}542 \mu \mathrm{g} / \mathrm{mL} \\
\text { (HNE) }\end{array}$ & & & & & {$[199,201]$} \\
\hline & & & & $\begin{array}{c}547.69 / 601.49 \\
\mu \mathrm{g} / \mathrm{mL} \\
(\mathrm{MPO})^{*}\end{array}$ & & & & & [349] \\
\hline & & & & & $<100 \mu \mathrm{g} / \mathrm{mL}$ & & & & [297] \\
\hline $\begin{array}{l}\text { H. procumbens } \\
\text { tincture }\end{array}$ & & & & $\begin{array}{c}915.55 / 776.49 \\
\mu \mathrm{g} / \mathrm{mL} \\
(\mathrm{MPO})^{*}\end{array}$ & & & & & [349] \\
\hline $\begin{array}{c}\text { Aqueous } \\
\text { ethanolic } H . \\
\text { zeyheri extract }\end{array}$ & & & & $\begin{array}{c}1012 \mu \mathrm{g} / \mathrm{mL} \\
(\mathrm{HNE})\end{array}$ & & & & & {$[199,201]$} \\
\hline \multirow[t]{2}{*}{$\begin{array}{l}\text { Aqueous } H \text {. } \\
\text { procumbens } \\
\text { extract }\end{array}$} & 8.9 & & & & & $0.55 \mu \mathrm{g} / \mathrm{mL}$ & & & [350] \\
\hline & 27 & & & & & $0.2 \mu \mathrm{g} / \mathrm{mL}$ & & & [350] \\
\hline $\begin{array}{l}\text { Ethanolic } H \text {. } \\
\text { procumbens } \\
\text { extract }\end{array}$ & & & & $\begin{array}{l}65.5 \mu \mathrm{g} / \mathrm{mL} \\
(\mathrm{FAAH})\end{array}$ & & & & & [304] \\
\hline $\begin{array}{l}\text { Ethyl acetate } \\
\text { fraction of } \\
\text { aqueous } \\
\text { ethanolic } H \text {. } \\
\text { procumbens } \\
\text { extract }\end{array}$ & 19.95 & $391 \mu \mathrm{g} / \mathrm{mL}$ & - & & & & & & [285] \\
\hline $\begin{array}{l}\text { Butanol } \\
\text { fraction of } \\
\text { aqueous } \\
\text { ethanolic } H \text {. } \\
\text { procumbens } \\
\text { extract }\end{array}$ & 19.5 & $565 \mu \mathrm{g} / \mathrm{mL}$ & $203 \mu \mathrm{g} / \mathrm{mL}$ & & & & & & [285] \\
\hline $\begin{array}{c}\text { Methanolic } H \text {. } \\
\text { procumbens } \\
\text { extract }\end{array}$ & & & & & & & & $1046 \mu \mathrm{g} / \mathrm{mL}$ & [295] \\
\hline $\begin{array}{l}\text { H. procumbens } \\
\text { extracts and } \\
\text { isolates }\end{array}$ & & & & & & & & $\sim 125 \mu \mathrm{g} / \mathrm{mL}$ & [296] \\
\hline $\begin{array}{c}\text { Isolated } \\
\text { compounds }\end{array}$ & & & & & & & & & \\
\hline \multirow[t]{8}{*}{ Harpagoside } & & $30 \mu \mathrm{M} / \mathrm{L}$ & $48.6 \mu \mathrm{M} / \mathrm{L}$ & & & & & & {$[332,333]$} \\
\hline & & $39 \mu \mathrm{M} / \mathrm{L}$ & $49 \mu \mathrm{M} / \mathrm{L}$ & & & & & & [285] \\
\hline & & & & & & & & $1041 \mu \mathrm{g} / \mathrm{mL}$ & [295] \\
\hline & & & & $\begin{array}{c}>600 \mu \mathrm{g} / \mathrm{mL} \\
(\mathrm{HNE})\end{array}$ & & & & & {$[199,201]$} \\
\hline & & & & $\begin{array}{l}92.7 \mu \mathrm{M} \\
(\mathrm{AChE})\end{array}$ & & & & & [351] \\
\hline & & & & $\begin{array}{l}95.6 \mu \mathrm{M} \\
\text { (AChE) }\end{array}$ & & & & & [351] \\
\hline & & & & & & & $96.4 \mu \mathrm{M}$ & & [293] \\
\hline & & & & & $14.04 \mu \mathrm{M}$ & & & & [302] \\
\hline Harpagide & & & & & & & & $1186 \mu \mathrm{g} / \mathrm{mL}$ & [295] \\
\hline \multirow[t]{2}{*}{ 8-PCHG } & & & & $\begin{array}{c}179 \mu \mathrm{g} / \mathrm{mL} \\
\text { (HNE) }\end{array}$ & & & & & {$[199,201]$} \\
\hline & & & & $\begin{array}{l}95.6 \mu \mathrm{M} \\
(\mathrm{AChE})\end{array}$ & & & & & [351] \\
\hline Pagoside & & & & $\begin{array}{c}154 \mu \mathrm{g} / \mathrm{mL} \\
\text { (HNE) }\end{array}$ & & & & & {$[199,201]$} \\
\hline Caffeic acid & & & & $\begin{array}{c}86 \mu \mathrm{g} / \mathrm{mL} \\
\text { (HNE) }\end{array}$ & & & & & {$[199,201]$} \\
\hline Acetoside & & & & $\begin{array}{c}>500 \mu \mathrm{g} / \mathrm{mL} \\
(\mathrm{HNE})\end{array}$ & & & & & {$[199,201]$} \\
\hline
\end{tabular}


Table 8. Cont

\begin{tabular}{|c|c|c|}
\hline & $\mathrm{IC}_{50}$ & Reference \\
\hline & $\begin{array}{c}19.9 \mu \mathrm{M} \\
\text { (AChE), 35 } \\
\mu \mathrm{M}(\mathrm{BChE})\end{array}$ & [351] \\
\hline \multirow[t]{2}{*}{ Isoacetocide } & $\begin{array}{c}179 \mu \mathrm{g} / \mathrm{mL} \\
\text { (HNE) }\end{array}$ & {$[199,201]$} \\
\hline & $\begin{array}{c}21.6 \mu \mathrm{M} \\
(\mathrm{AChE}), 29.7 \\
\mu \mathrm{M}(\mathrm{BChE})\end{array}$ & [351] \\
\hline Decaffeoylverbascoside & $\begin{array}{c}16.1 \mu \mathrm{M} \\
(\mathrm{AChE}), 46 \\
\mu \mathrm{M}(\mathrm{BChE})\end{array}$ & [351] \\
\hline $\begin{array}{c}6^{\prime}-\mathrm{O}- \\
\text { Acetylacteosid }\end{array}$ & $\begin{array}{c}47 \mu \mathrm{g} / \mathrm{mL} \\
\text { (HNE) }\end{array}$ & {$[199,201]$} \\
\hline
\end{tabular}

* Formyl methionyl leucine phenylalanine- and arachidonic acid-stimulated, respectively.

Table 9 summarizes the results of pre-clinical experiments which studied other effects of Harpagophytum and its compounds.

Table 9. Experiments regarding other effects of devil's claw preparations and compounds.

\begin{tabular}{|c|c|c|c|c|}
\hline Effect & Study & Type & Year & Reference \\
\hline \multirow[t]{6}{*}{ Antioxidant } & $\begin{array}{l}\text { Rats, Harpagophytum * extract, } 100 \text { and } 200 \mathrm{mg} / \mathrm{kg} \text { bw or } \\
\text { selegiline i.p. for } 1,7 \text {, or } 14 \text { days; dose-dependent increase of } \\
\text { superoxide dismutase, catalase, and glutathione peroxidase } \\
\text { activities and reduction of lipid peroxidase similar to selegiline } \\
\text { after } 7 \text { days. }\end{array}$ & In Vivo & 1998 & $\begin{array}{l}\text { Bhattacharya and } \\
\text { Bhattacharya [352] }\end{array}$ \\
\hline & $\begin{array}{l}\text { Luminol-enhanced chemiluminescence in a xanthine/xanthine } \\
\text { oxidase cell-free system; Harpagophytum root powder; } \\
\text { superoxide and peroxyl were scavenged dose-dependently. }\end{array}$ & In Vitro & 2002 & Langmead et al. [353] \\
\hline & $\begin{array}{l}\text { Trolox equivalent antioxidant capacity (TEAC) assay; } \\
\text { Harpagophytum aqueous extract }(2.6 \% \text { harpagoside) and } \\
\text { harpagoside; extract rich in water-soluble antioxidants, } \\
\text { harpagoside showed poor activity. }\end{array}$ & In Vitro & 2003 & $\begin{array}{c}\text { Betancor-Fernandez } \\
\text { et al. [354] }\end{array}$ \\
\hline & $\begin{array}{l}\text { Rat renal mesangial cells; IL- } 1 \beta \text {-induced NO formation and } \\
\text { transcriptional regulation of iNOS; } H \text {. procumbens extracts with } \\
\text { varying harpagoside content and pure harpagoside; } \\
\text { dose-dependent and harpagoside-independent inhibition of } \\
\text { iNOS expression. }\end{array}$ & In Vitro & 2004 & Kaszkin et al. [350] \\
\hline & $\begin{array}{l}\text { Harpagophytum aqueous extract; protection from } \\
\text { DNA-damaging effects of stannous chloride in proficient and } \\
\text { deficient E. coli model; possible chelating, scavenger, or oxidant } \\
\text { activity postulated. }\end{array}$ & In Vitro & 2007 & Almeida et al. [355] \\
\hline & $\begin{array}{l}\text { Antioxidant characteristics using in vitro test systems, DPPH } \\
\text { radical scavenging, stimulated nitrite generation, neutrophil } \\
\text { superoxide anion generation, and neutrophil myeloperoxidase } \\
\text { (MPO); Harpagophytum extract (1.2\% harpagoside), tincture, } \\
\text { harpagoside; dose-dependent effect in all models, minimal } \\
\text { scavenging activity of harpagoside. }\end{array}$ & In Vitro & $\begin{array}{l}2005 \\
2009\end{array}$ & $\begin{array}{l}\text { Grant et al. [349], } \\
\text { Grant [356] }\end{array}$ \\
\hline
\end{tabular}

Antioxidant activities of total methanol extracts, fractions

(phenylethanoids, terpenoids, and sugars), and

$\beta$-OH-verbascoside, verbascoside, and leucosceptoside from cell suspension culture of $H$. procumbens; DPPH, superoxide anion generation, and oxygen radical absorbance capacity (ORAC)

In Vitro $2010 \quad$ Georgiev et al. [357] assays; $\beta-\mathrm{OH}$-verbascoside most active in $\mathrm{DPPH}$ and superoxide anion generation, leucosceptoside in ORAC.

Ferric-reducing antioxidant power test; $H$. procumbens crude methanolic extract, phenylethanoid-containing fraction, and verbascoside; strong ferrous ion-chelating capacity. 
Table 9. Cont.

\begin{tabular}{|c|c|c|c|c|}
\hline Effect & Study & Type & Year & Reference \\
\hline & $\begin{array}{l}\text { Brain homogenates, catalase activity and thiol levels, brain } \\
\text { cortical slices; lipid peroxidation, antioxidant defenses, cell } \\
\text { damage, respectively; H. procumbens infusion, crude extract, } \\
\text { and fractions; dose-dependent inhibition of lipid peroxidation, } \\
\text { ethyl acetate fraction had the highest antioxidant effects. }\end{array}$ & In Vitro & 2013 & $\begin{array}{l}\text { Schaffer et al. } \\
\quad[359,360]\end{array}$ \\
\hline & $\begin{array}{l}\text { Human neutrophils challenged with phorbol myristate acetate } \\
\text { (PMA), opsonized Staphylococcus aureus, and Fusobacterium } \\
\text { nucleatum; } 5 \text { taxa of Harpagophytum, including one hybrid; high } \\
\text { variability in suppression of respiratory burst, hybrid with } \\
\text { highest antioxidant capacity but proinflammatory effect, three } \\
\text { taxa with anti-inflammatory effect. }\end{array}$ & In Vitro & 2016 & Muzila et al. [361] \\
\hline & $\begin{array}{l}\text { Adult male Wistar rats, fluphenazine-induced orofacial dyskinesia } \\
\text { (OD); DPPH assay; ethyl acetate fraction of H. procumbens (10, 30, } \\
\text { or } 100 \mathrm{mg} / \mathrm{kg} \text { i.p.); inhibition of vacuous chewing movements, } \\
\text { decreased locomotion unchanged, protective against change in } \\
\text { catalase activity, not against ROS increase. }\end{array}$ & In Vivo & 2016 & $\begin{array}{l}\text { Schaffer et al. } \\
\text { [362] }\end{array}$ \\
\hline & $\begin{array}{l}\text { Porcine neutrophils; respiratory burst; harpagoside; significant } \\
\text { inhibition of ROS production. }\end{array}$ & In Vitro & 2017 & Mosca et al. [363] \\
\hline & $\begin{array}{l}\text { Male Sprague-Dawley rats, modified rodent contusion model } \\
\text { of spinal cord injury, murine BV-2 microglial cells; } H \text {. } \\
\text { procumbens hydroethanolic extract ( } 5.3 \% \text { harpagoside, } 300 \\
\text { mg/kg); behavioral and neurochemical parameters, improved, } \\
\text { some significantly, in cell line, oxidative stress and } \\
\text { inflammatory response were suppressed. }\end{array}$ & $\begin{array}{l}\text { In Vitro and } \\
\text { in vivo }\end{array}$ & 2020 & $\begin{array}{l}\text { Ungerer et al. } \\
\text { [364] }\end{array}$ \\
\hline
\end{tabular}

LPS-induced RAW 264.7 mouse and U937 human

macrophages; DPPH and 2,2'-azino-bis

(3-ethylbenzothiazoline-6-sulphonic acid) (ABTS) assays;

aqueous, ethanolic, and ethyl acetate extracts of H. zeyheri; for $\quad$ In Vitro 2021 Ncube et al. [365]

all extracts, dose-dependent inhibition of IL-10 expression,

In Vitro 2021 Ncube et al. [365]

ethyl acetate fraction with lowest $\mathrm{IC}_{50}$ in both assays, $\mathrm{NO}$ and TNF- $\alpha$ inhibition similar to diclofenac.

\begin{tabular}{|c|c|c|c|c|}
\hline Antidiabetic & $\begin{array}{l}\text { Streptozotocin-induced diabetes mellitus in rats; H. procumbens } \\
\text { root aqueous extract }(50-800 \mathrm{mg} / \mathrm{kg} \text { i.p.); significant reduction } \\
\text { in blood glucose levels in normal and diabetic rats. }\end{array}$ & In Vitro & 2004 & $\begin{array}{l}\text { Mahomed and } \\
\text { Ojewole [317], } \\
\text { Mahomed [318] }\end{array}$ \\
\hline \multirow[t]{3}{*}{ Anticholinesterase } & $\begin{array}{l}\text { Chick, guineapig, and rabbit isolated gastro-intestinal smooth } \\
\text { muscle preparations; } H \text {. procumbens root aqueous extract } \\
(10-1000 \mu \mathrm{g} / \mathrm{mL}) ; \text { dose-dependent contractions of } \\
\text { gastro-intestinal tract smooth muscles. }\end{array}$ & In Vitro & 2005 & $\begin{array}{l}\text { Mahomed [318], } \\
\text { Mahomed et al. } \\
\quad[366]\end{array}$ \\
\hline & $\begin{array}{l}\text { Spectrophotometric method using acetylthiocholine and } \\
\text { butyrylcholine chloride as substrates; H. procumbens crude } \\
\text { methanolic extract, phenylethanoid-containing fraction, and } \\
\text { verbascoside; significant cholinesterase inhibitory activity. }\end{array}$ & In Vitro & 2011 & $\begin{array}{l}\text { Georgiev et al. } \\
{[358]}\end{array}$ \\
\hline & $\begin{array}{l}\text { Spectrophotometric method, acetylcholinesterase (AchE) and } \\
\text { butyrylcholinesterase (BchE) inhibition; H. procumbens ethyl } \\
\text { acetate extract } \\
\text { and fractions; inhibition by verbascosides }>60 \%\end{array}$ & In Vitro & 2013 & Bae et al. [351] \\
\hline \multirow[t]{2}{*}{ Antimicrobial } & $\begin{array}{l}\text { Harpagophytum extract (not specified) showed mild antifungal } \\
\text { effects against Penicillum digitatum and Botrytis cinerea. }\end{array}$ & In Vitro & 1985 & $\begin{array}{l}\text { Guérin and } \\
\text { Réveillère [367] }\end{array}$ \\
\hline & $\begin{array}{l}\text { Harpagophytum dry extract ( } 2.6 \% \text { harpagoside) and harpagoside; } \\
\text { inhibition of a panel (all) of aerobic bacteria, C. krusei, and two } \\
\text { anaerobic bacteria strains, harpagoside without effect. }\end{array}$ & In Vitro & 2007 & $\begin{array}{l}\text { Weckesser et al. } \\
\text { [368] }\end{array}$ \\
\hline
\end{tabular}


Table 9. Cont.

\begin{tabular}{|c|c|c|c|c|}
\hline Effect & Study & Type & Year & Reference \\
\hline & $\begin{array}{l}\text { Chloroquine (CQ)-sensitive and CQ-resistant strains of } P \text {. } \\
\text { falciparum, and cytotoxicity in CHO and HepG2 cells; extracts } \\
\text { of } H \text {. procumbens aerial parts and seeds, and petrol ether of the } \\
\text { root, (+)-8,11,13-totaratriene- } 12,13 \text {-diol and ferruginol, and CQ } \\
\text { diphosphate as control; the two diterpenes showed significant } \\
\text { inhibition of both strains without being cytotoxic. }\end{array}$ & In Vitro & 2003 & $\begin{array}{l}\text { Clarkson et al. } \\
\text { [200] }\end{array}$ \\
\hline & $\begin{array}{l}\text { Female Balb/c mice, infected with Toxocara canis; } \\
\text { Harpagophytum ethanolic extract }(100 \mathrm{mg} / \mathrm{kg}) \text {; decrease in } \\
\text { eosinophil accumulation, IL-5 and IgE significantly decreased. }\end{array}$ & In Vivo & $\begin{array}{l}2012 \\
2014\end{array}$ & $\begin{array}{l}\text { Oliveira et al. } \\
\text { [369-371] }\end{array}$ \\
\hline & $\begin{array}{l}\text { Harpagophytum ethanolic extract showed dose-dependent effect } \\
\text { on Schistosoma mansoni, mechanism of action proposed; proteins } \\
\text { relevant for cellular homeostasis identified as possible targets. }\end{array}$ & In Vitro & 2014 & Correia [372] \\
\hline & $\begin{array}{l}\text { Bacterial triggers of rheumatoid arthritis, ankylosing } \\
\text { spondylitis, multiple sclerosis, and rheumatic fever; powdered } \\
\text { Harpagophytum extracts, various solvents; inhibition of Proteus } \\
\text { mirabilis, Klebsiella pneumoniae, Acinetobacter baylyi, } \\
\text { Pseudomonas aeruginosa, and Streptococcus pyogenes throughout, } \\
\text { methanolic extract more potent, no toxicity in Artemia nauplii } \\
\text { bioassay. (Note: throughout the publication, the substance of } \\
\text { investigation is mislabeled as devil's claw fruit, while it was, in } \\
\text { fact, the root being investigated (pers. comm. Ian Cock, 2021)) }\end{array}$ & In Vitro & 2017 & $\begin{array}{c}\text { Cock and } \\
\text { Bromley [373] }\end{array}$ \\
\hline Antimutagenic & $\begin{array}{l}\text { Cultured human lymphocytes; mutagenic activity of } \\
\text { 1-nitropyrene (1-Npy) in cytokinesis-block micronucleus assay; } \\
\text { Harpagophytum aqueous-ethanolic extract, harpagoside; } \\
\text { genotoxicity significantly reduced for both, only harpagoside } \\
\text { significantly reduced the mutagenicity of 1-Npy. }\end{array}$ & In Vitro & $\begin{array}{l}2014 \\
2015\end{array}$ & $\begin{array}{c}\text { Luigi [374], } \\
\text { Luigi et al. [375] }\end{array}$ \\
\hline \multirow[t]{3}{*}{ Anti-osteoporotic } & $\begin{array}{l}\text { Male ICR mice, female C57BL/6J mice; receptor activator of } \\
\text { nuclear factor k-B ligand (RANKL)-induced osteoclastogenesis; } \\
\text { harpagoside; inhibition of RANKL, osteoclast formation, and } \\
\text { LPS-induced bone loss, but not ovariectomy-mediated } \\
\text { bone erosion. }\end{array}$ & In Vitro & 2015 & Kim et al. [376] \\
\hline & $\begin{array}{l}\text { Mouse calvaria MC3T3-E1cells; bone formation and } \\
\text { resorption, bone-loss in ovariectomized (OVX) mouse model; } \\
\text { harpagide; stimulated differentiation and maturation of } \\
\text { osteoblast cells and suppressed RANKL-induced } \\
\text { osteoclastogenesis, improved bone recovery in OVX model, } \\
\text { inhibited markers of bone loss in the serum. }\end{array}$ & $\begin{array}{l}\text { In Vitro and } \\
\text { in vivo }\end{array}$ & 2016 & Chung et al. [377] \\
\hline & $\begin{array}{l}\text { Mouse calvaria MC3T3-E1cells; bone formation and resorption, } \\
\text { bone-loss in ovariectomized (OVX) mouse model; harpagoside; } \\
\text { stimulated differentiation and maturation of osteoblast cells and } \\
\text { suppressed RANKL-induced osteoclastogenesis, improved bone } \\
\text { recovery in OVX model, inhibited markers of bone loss in } \\
\text { the serum. }\end{array}$ & $\begin{array}{l}\text { In Vitro and } \\
\text { in vivo }\end{array}$ & 2017 & Chung et al. [378] \\
\hline \multirow[t]{2}{*}{ Cardiovascular } & $\begin{array}{l}\text { Frog and guineapig hearts, cats; cardiac muscle contraction } \\
\text { and blood pressure, dose-dependent positive and negative } \\
\text { inotropic effects, no effect on blood pressure. }\end{array}$ & $\begin{array}{l}\text { In Vitro and } \\
\text { in vivo }\end{array}$ & 1965 & Vollmann [379] \\
\hline & $\begin{array}{l}\text { Normotensive rats, rabbit heart; methanolic extract of } \\
\text { Harpagophytum, harpagoside, harpagide; decrease in blood } \\
\text { pressure and heart rate observed, less with harpagoside; } \\
\text { extract mild inotropic at lower and negative inotropic at } \\
\text { higher doses, harpagoside more negative chronotropic and } \\
\text { positive inotropic, harpagide only slightly negative } \\
\text { chronotropic but considerably negative inotropic. }\end{array}$ & $\begin{array}{l}\text { In Vitro and } \\
\text { in vivo }\end{array}$ & 1984 & $\begin{array}{l}\text { Circosta et al. } \\
\text { [380] }\end{array}$ \\
\hline
\end{tabular}


Table 9. Cont.

\begin{tabular}{|c|c|c|c|c|}
\hline Effect & Study & Type & Year & Reference \\
\hline & $\begin{array}{l}\text { Rat heart; methanolic extract of Harpagophytum }(8.5 \% \\
\text { harpagoside and } 10.5 \% \text { total iridoids) and harpagoside; } \\
\text { significant, dose-dependent, protective action toward } \\
\text { hyperkinetic ventricular arrhythmias. }\end{array}$ & In Vitro & 1985 & $\begin{array}{l}\text { De Pasquale et al. } \\
\text { [381] }\end{array}$ \\
\hline & $\begin{array}{l}\text { Langendorff preparations of rat heart; ischemic perfusion induced } \\
\text { hyperkinetic ventricular arrhythmia; H. procumbens, harpagoside; } \\
\text { significant, dose-dependent protective action for both. }\end{array}$ & In Vitro & 1985 & $\begin{array}{l}\text { De Pasquale et al. } \\
\text { [382] }\end{array}$ \\
\hline & $\begin{array}{l}\text { Guineapig ileum and rabbit jejunum; Harpagophytum extract, } \\
\text { harpagoside, harpagide; spasmolytic effect, strongest for } \\
\text { harpagoside. }\end{array}$ & In Vitro & 1985 & $\begin{array}{l}\text { Occhiuto et al. } \\
\text { [383] }\end{array}$ \\
\hline & $\begin{array}{l}\text { Dogs; harpagoside, harpagide }(3.4 \mathrm{mg} / \mathrm{kg}) \text {; decrease of mean } \\
\text { aortic pressure with harpagoside. }\end{array}$ & In Vivo & 1990 & $\begin{array}{l}\text { Occhiuto and de } \\
\text { Pasquale [384] }\end{array}$ \\
\hline & $\begin{array}{l}\text { Multiple mammalian animal models; } H \text {. procumbens root } \\
\text { aqueous extract }(10-400 \mathrm{mg} / \mathrm{kg} \text { i.v., } 10-1000 \mu \mathrm{g} / \mathrm{mL}) ; \\
\text { dose-dependent, significant hypotensive, cardio-depressant, } \\
\text { and vasorelaxant effects. }\end{array}$ & $\begin{array}{l}\text { In Vitro and } \\
\text { in vivo }\end{array}$ & 2004 & $\begin{array}{l}\text { Mahomed and } \\
\text { Ojewole [385], } \\
\text { Mahomed [318] }\end{array}$ \\
\hline \multirow[t]{4}{*}{ Neuroprotective } & $\begin{array}{l}\text { Pentylenetetrazole (PTZ)-, picrotoxin (PCT)-, and bicuculline } \\
\text { (BCL)-induced seizures in mice; } H \text {. procumbens aqueous extract } \\
(100-800 \mathrm{mg} / \mathrm{kg} \text { i.p.); PZT-induced seizures significantly } \\
\text { reduced, PCT and BCL to a lesser extent, CNS depressed. }\end{array}$ & In Vivo & 2006 & $\begin{array}{l}\text { Mahomed and } \\
\text { Ojewole [386] }\end{array}$ \\
\hline & $\begin{array}{l}\text { Rat hypothalamic (Hypo-E22) cells and rat cortex challenged } \\
\text { with amyloid } \beta \text {-peptide; } H \text {. procumbens aqueous extract; } \\
\text { increased brain-derived neurotrophic factor gene expression and } \\
\text { decreased TNF- } \alpha \text { gene expression in Hypo-E22 cells, alleviated } \\
\text { decreased monoaminergic signaling in cortex presynaptic } \\
\text { endings. }\end{array}$ & $\begin{array}{l}\text { In Vitro and } \\
\text { ex vivo }\end{array}$ & 2017 & $\begin{array}{l}\text { Ferrante et al. } \\
\quad[387]\end{array}$ \\
\hline & $\begin{array}{l}\text { Male Wistar rats; chronic cerebral hypoperfusion model; } \\
\text { harpagoside ( } 15 \mathrm{mg} / \mathrm{kg} \text {, } 60 \text { days); symptoms of vascular } \\
\text { dementia spatial and fear memory impairments restored, } \\
\text { phosphatase and tensin homolog (PTEN) significantly } \\
\text { suppressed. }\end{array}$ & In Vivo & 2018 & Chen et al. [388] \\
\hline & $\begin{array}{l}\text { Female Wistar albino rats, arsenic induced neurotoxicity; } \\
\text { Harpagophytum powder ( } 200 \text { and } 400 \mathrm{mg} / \mathrm{kg} \text {, p.o.); behavioral } \\
\text { and biochemical parameters improved significantly. }\end{array}$ & In Vivo & 2020 & Peruru et al. [389] \\
\hline $\begin{array}{l}\text { Immunomodulatory/ } \\
\text { thymomimetic }\end{array}$ & $\begin{array}{l}\text { Maturation of mice thymocytes in the presence of a } \\
\text { glycocorticosteroid, cytotoxicity by microscopy and flow } \\
\text { cytometry; ethanolic extract of Harpagophytum, Filipendula } \\
\text { ulmaria, and Echinacea purpurea, various dilutions; } 17 \% \\
\text { increase in the number of surviving cells. }\end{array}$ & In Vitro & 2002 & $\begin{array}{l}\text { Prosinska et al. } \\
\text { [390] }\end{array}$ \\
\hline \multirow[t]{2}{*}{ Anorexigenic } & $\begin{array}{l}\text { Male C57BL/ } 6 \text { mice, calcium mobilization and growth } \\
\text { hormone secretagogue receptor (GHS-R1a) internalization; } \\
\text { Harpagophytum root powder; significantly increased cellular } \\
\text { calcium influx but no induction of GHS-R1a receptor } \\
\text { internalization, significant anorexigenic effect. }\end{array}$ & In Vivo & 2014 & $\begin{array}{l}\text { Torres-Fuentes } \\
\text { et al. [391] }\end{array}$ \\
\hline & $\begin{array}{l}\text { Male Wistar rats; obestatin secretion; Harpagophytum } \\
\text { hydroalcoholic extract }(150,300 \text {, and } 600 \mathrm{mg} / \mathrm{kg}) ; \text { significantly } \\
\text { increased serum levels of obestatin and reduced body weight } \\
\text { at } 300 \text { and } 600 \mathrm{mg} / \mathrm{kg} \text {. }\end{array}$ & In Vivo & 2016 & Saleh et al. [392] \\
\hline Metal accumulation & $\begin{array}{l}\text { Rats, supplemented with lead acetate; Harpagophytum infusion } \\
(30 \mathrm{mg} / \mathrm{kg}) ; \text { significant reduction of lead deposits }\end{array}$ & In Vitro & 1975 & $\begin{array}{l}\text { Int. Bio Research } \\
\text { [393] }\end{array}$ \\
\hline
\end{tabular}

* Species not specified; however, all specific attribution must be cautioned against due to the frequent admixture. 
Primary—anti-inflammatory, analgesic/antinociceptive, and antioxidant—effects have been demonstrated in multiple in vitro, in vivo, and ex vivo assays with crude extracts, fractions, and isolated compounds of Harpagophytum. However, experiments show some inconsistencies, likely caused by deviations in experimental models and insufficient characterization of the purportedly active compounds, as well as variation in solvent systems [394-396]. Further, the consolidated data show that efficacy cannot be clearly attributed to any one of the compounds present in Harpagophytum. Focus on harpagoside-albeit serving as a convenient marker-cannot be substantiated in an efficacy context. On the other hand, the presence and effect of verbascoside in Harpagophytum, a compound with well-documented anti-inflammatory properties, has not been adequately studied.

\subsection{Pharmacokinetics}

Most of the available pharmacokinetics data were created as a byproduct or in the context of pharmacological experiments with Harpagophytum preparations or its compounds. Vanhaelen [52] experimented with harpagoside and harpagide under conditions mimicking those found in the stomach and concluded by suggesting enteric-coated preparations for harpagoside to slow down acid hydrolysis. Chrubasik [217] investigated release and stability of harpagoside in gastric and intestinal fluids and stability for 3 and $6 \mathrm{~h}$, respectively. The author also found harpagoside to be of low bioavailability, a daily dose of $100 \mathrm{mg}$ could not be detected in serum or urine. Chrubasik et al. (2000) [238] established an octanol-water distribution coefficient of approximately 4 that is not dependent on $\mathrm{pH}$ or temperature.

Neither Harpagophytum ethanolic extract nor harpagoside had a relevant effect on cytochrome P (CYP) 450 3A4 in vitro [397]. An investigation of different Harpagophytum extracts elucidated maximum levels of plasma harpagoside after 1.3 to $2.5 \mathrm{~h}$ and suggested a correlation between serum harpagoside levels and inhibition of leukotriene biosynthesis in vitro and ex vivo [285,398]. In human liver microsomes and subtype-specific CYP substrates, Harpagophytum at a dose derived from [157] activated CYP 2E1 and inhibited CYP 2C19 [399]. Inhibition of CYP 450 was shown for methanolic extracts of $\mathrm{H}$. procumbens, and while inhibition of CYP 1A2 and 2D6 was relatively low, moderate inhibition of CYP 2C8/9/19 and 3A4 was noted ( $\mathrm{IC}_{50}$ between 100 and $350 \mu \mathrm{g} / \mathrm{mL}$ ) [400]. However, the impact on drugs metabolized via those enzymes is merely theoretical. Romiti et al. [401] found Harpagophytum to interact with the multidrug transporter ABCB1/P-glycoprotein, unrelated to relative harpagoside content. Modarai et al. [402] found Harpagophytum preparations, but not harpagoside or harpagide, to weakly inhibit CYP 3A4, but deemed clinical relevance unlikely.

\subsection{Toxicology}

Acute and chronic toxicity have been investigated for the herbal substance, its preparations, and compounds isolated from Harpagophytum. Multiple publications cite an unpublished experiment by Albus (1958) in which an $\mathrm{LD}_{50}$ in mice was established for a liquid extract (not specified) at $34 \mathrm{~mL} / \mathrm{kg}$ i.v. and $220 \mathrm{~mL} / \mathrm{kg}$ p.o. [22,42,51,120]. An $\mathrm{LD}_{50}$ in rats was given at $10 \mathrm{~g} / \mathrm{kg}$ for a spray extract and in mice at $1 \mathrm{~g} / \mathrm{kg}$ for harpagoside [403]. Vollmann [379] established an $\mathrm{LD}_{50}$ of 23 and $10 \mathrm{~mL} / \mathrm{kg}$ for an infusion and a chloroform/butanolic extract (4:1), respectively. Möse [404], in an unpublished report (cited in $[27,44,67,405]$ ) conducted toxicity tests with a Harpagophytum infusion in primate and chicken tissue cultures, and no effect on cell development was found, nor did the infusion promote growth of Ehrlich ascites carcinoma in mice. Eichler and Koch referenced toxicity at above $0.5 \mathrm{~g} / \mathrm{kg}$ without citation [305]. Erdös and colleagues [308] demonstrated Harpagophytum aqueous, methanolic, and butanolic extracts to be effectively non-toxic $\left(\mathrm{LD}_{0}\right.$ at $4640 \mathrm{mg} / \mathrm{kg}$ p.o. and $>1000 \mathrm{mg} / \mathrm{kg}$ i.v.), and for harpagoside, a $\mathrm{LD}_{0}$ of $395 \mathrm{mg} / \mathrm{kg}$ and a $\mathrm{LD}_{50}$ of $511 \mathrm{mg} / \mathrm{kg}$. Marzin (1978, cited in [67]) confirmed these results. The same author investigated the toxicity of an extract (2.7\% total iridoids), p.o. or i.p., in rats and mice. Administration p.o. was effectively non-toxic, while i.p., some toxicity was observed with 
a calculated $\mathrm{LD}_{50}$ of $10 \mathrm{~g} / \mathrm{kg}$ (Marzin, 1981, cited in [274]). Vanhalen and colleagues tested toxicity of harpagoside and harpagide in mice and established an $\mathrm{LD}_{50}$ of 1 and $3.2 \mathrm{~g} / \mathrm{kg}$, respectively [224]. Schmidt [44] elaborated unpublished toxicological investigations with Harpagophytum D2 and Harpagosan (DER 2:1) [406,407], establishing an $\mathrm{LD}_{50}$ of $20 \mathrm{~mL} / \mathrm{kg}$ and $>30 \mathrm{mg} / \mathrm{kg}$, respectively. Whitehouse et al. [341] established an $\mathrm{LD}_{50}$ at $13.5 \mathrm{~g} / \mathrm{kg}$ p.o. for a Harpagophytum root extract (not specified) in mice and no toxicity at $7.5 \mathrm{~g} / \mathrm{kg}$ over three weeks in rats, while $2 \mathrm{~g} / \mathrm{kg}$ over one week showed no impact on liver parameters. Ibrahim et al. [408] conducted a battery of toxicity studies in mice (acute, sub-acute, and chronic) with a commercial product (Boiron, France-composition not declared) and found no clinically relevant changes in any of the tested outcomes, attributing a slight increase in liver enzymes to the anti-inflammatory effect. Al-Harbi and colleagues [409] found no oral acute toxicity in mice at 1 and $3 \mathrm{~g} / \mathrm{kg}$ Harpagophytum powder. In a 90-day chronic toxicity study (test substance not characterized), no clinically relevant changes in tested parameters were established, except for a significant decrease in blood sugar and uric acid levels. Both chronic assessments, however, must be considered inadequate due to the insufficiently characterized test material. Allard et al. [410] discussed herb-induced nephrotoxicity, and in that context, called for further investigation of whether a theoretical impact of Harpagophytum on major renal transport processes is of clinical relevance. Joshi et al. [411] investigated the toxicology of a $H$. procumbens aqueous-ethanolic extract $(1 \mathrm{~g} / \mathrm{kg} /$ day, equivalent to $7.5-10 \times$ the human recommended dose) in male and female Sprague Dawley rats over 4 and 12 weeks. While no significant histopathological effects were found, the study yielded significant-albeit not clinically relevant-sex-related differences in blood chemistry. All these results stand in stark contrast to those of Zorn [116], casting considerable doubt over the authenticity of the plant material used in his experiments.

Mahomed and Ojewole [412,413] conducted experiments in vitro suggesting spasmogenic and uterotonic actions for an $\mathrm{H}$. procumbens aqueous extract $(10-1000 \mu \mathrm{g} / \mathrm{mL})$. Whether these results are of clinical relevance in vivo remains to be established (see Section 11.2). Pearson [414] studied the reproductive toxicity of a combination product containing Harpagophytum (exact composition not disclosed) for veterinary use in pregnant female Sprague Dawley rats and showed no signs of toxicity. The study, however, is poorly reported and of limited relevance given the unknown composition of the test substance. Contrarily, Davari and colleagues [415] reported teratogenic effects and histopathological changes in fetal tissues (but no significant structural malformations or abnormalities) from an experiment with $H$. procumbens $(200,400,600 \mathrm{mg} / \mathrm{kg})$ in pregnant Balb/C mice.

\section{Clinical Research}

\subsection{Efficacy}

The efficacy of devil's claw has been investigated in more than 50 human studies, and case reports and observational studies are summarized in Table 10, while randomized, controlled trials (RCTs) are summarized in Table 11. Indications were primarily degenerative joint diseases as well as low back pain. Trials utilized a variety of methodological designs, with different preparations of devil's claw and daily doses of harpagoside, varying from $<30$ to $>100 \mathrm{mg}$. While harpagoside is considered to contribute to the overall activity of devil's claw preparations, it is not yet fully understood which other compounds may also be of relevance. Furthermore, an investigation into the harpagoside content of commercially available devil's claw preparations revealed substantial variation, with contents often below the recommended daily dose of $4.5-9 \mathrm{~g}$ crude drug (equivalent $>50 \mathrm{mg}$ harpagoside) $[173,232-234,237,416]$.

Trials have been reviewed systematically with regards to their quality and results concerning safety and efficacy of Harpagophytum preparations in publications between 1973 and $2019[17,23,139,156,417-433]$. Another set of reviews considered the efficacy of devil's claw preparations or its active compounds in specific need states [23,130,434-488]. All trials observed improvement of the outcome criteria under treatment (some significant), however, significant superiority of the Harpagophytum preparations vs. conventional NSAIDs was 
not reported. This is partly because most trials were observational and/or comparative, while the outcomes of placebo-controlled trials were often inconclusive or overshadowed by methodological deficiencies. Many trials allowed for conventional emergency or comedication, which further limits the value of the data collected. Despite some studies providing evidence for the effectiveness of certain preparations, the overall quality of evidence is not sufficient. Furthermore, the relevance of early studies with homeopathic dilutions - while included here for completeness' sake-is limited from a perspective of rational phytotherapy.

Table 10. Case reports and observational studies conducted between 1971 and 2021.

\begin{tabular}{|c|c|c|c|c|}
\hline Indication & $\begin{array}{l}\text { Trial Type, } \\
\text { Size }\end{array}$ & Results & Year & Reference \\
\hline Chemosis & $\begin{array}{c}\mathrm{CR} \\
1\end{array}$ & $\begin{array}{l}\text { Initial treatment with multiple preparations that did not lead } \\
\text { to improvement, then with } 300 \mathrm{mg} \text { Harpagophytum extract } \\
\text { (not specified) } 3 \text { times daily, orally, for } 6 \text { months, leading to } \\
\text { drastic improvement. }\end{array}$ & 1983 & Belaiche [489] \\
\hline $\begin{array}{l}\text { Familial } \\
\text { Mediterranean fever }\end{array}$ & $\begin{array}{l}\text { CR } \\
17\end{array}$ & $\begin{array}{l}\text { Harpagophytum extracts characterized as aqueous (DER } 1: 2.4 \text {, } \\
2.5 \% \text { harpagoside) - this characterization may also apply to } \\
\text { previous trials by Belaiche and Dahout (see above) - } 6-9 \mathrm{~g} \\
\text { single dose, duration not provided; significantly decreased } \\
\text { recurrence in } 80 \% \text { of patients. }\end{array}$ & 1983 & Belaiche [490] \\
\hline Cancer & $\begin{array}{c}\mathrm{CR} \\
2\end{array}$ & $\begin{array}{l}\text { Tumor regression after taking Harpagophytum extract ( } 500 \mathrm{mg} \\
\text { daily) and/or Essiac respectively, without cytotoxic therapy. }\end{array}$ & 2009 & Wilson [491] \\
\hline DJD & $\begin{array}{l}\mathrm{O} \\
\sim 120\end{array}$ & $\begin{array}{l}\text { Harpagophytum D4-D6, IA, and D1 orally; } 1-6 \text { months; } \\
\text { substantial improvement of symptoms in most cases. }\end{array}$ & 1971 & Beham [492] \\
\hline $\mathrm{CP}$ & $\begin{array}{l}\mathrm{O} \\
60\end{array}$ & $\begin{array}{l}\text { Harpagophytum D2, IA, plus tea ( } 2-3 \text { tsp per } 1 \mathrm{~L} \text { water) or } \\
3 \times 2 \text { tablets orally, duration not provided; dose-dependent } \\
\text { response; } 60 \% \text { substantial improvement of symptoms, } 20 \% \\
\text { improvement, } 20 \% \text { no change. }\end{array}$ & 1972 & Schmidt [43] \\
\hline CP, DJD & $\begin{array}{c}\mathrm{O} \\
146\end{array}$ & $\begin{array}{l}\text { Harpagophytum D2, IA, duration not provided; improvement } \\
\text { in } 134 \text { patients. }\end{array}$ & 1972 & $\begin{array}{l}\text { Zimmermann, } \\
\text { cited in [130] }\end{array}$ \\
\hline DJD & $\begin{array}{l}\mathrm{O} \\
25\end{array}$ & $\begin{array}{l}\text { Harpagophytum D2-D3, IA, and SC, 1-2 mL, pain-free after } \\
6 \text { injections, or tea ( } 1 \text { tsp per } 300 \mathrm{~mL} \text { ) daily for 3-6 weeks. }\end{array}$ & 1972 & Brantner [493] \\
\hline DJD & $\begin{array}{l}\mathrm{O} \\
70\end{array}$ & $\begin{array}{l}\text { Harpagophytum D2, IA, some + tea, some + indometacin, } \\
\text { duration not provided; improvement in } 90 \% \text { of patients. }\end{array}$ & 1976 & $\begin{array}{l}\text { Wilhelmer, cited } \\
\quad \text { in [44] }\end{array}$ \\
\hline CP, DJD & $\begin{array}{c}\mathrm{O} \\
21+\end{array}$ & $\begin{array}{l}\text { Harpagophytum D1-D3, IA, SC, and i.v., tea, orally, duration } \\
\text { not provided; significant improvement in } 30 \% \text { of patients. }\end{array}$ & 1977 & Zimmermann \\
\hline DJD & $\begin{array}{l}\mathrm{O} \\
84\end{array}$ & $\begin{array}{l}250 \text { or } 500 \mathrm{mg} \text { Harpagophytum extract (not specified) } 3 \text { times } \\
\text { daily orally for } 2-6 \text { months, improvement in } 72 \% \text { of patients. }\end{array}$ & 1979 & $\begin{array}{l}\text { Dahout, cited in } \\
\text { [495] }\end{array}$ \\
\hline CP, DJD & $\begin{array}{l}O \\
600\end{array}$ & $\begin{array}{l}\text { Harpagosan tea ( } 2 \text { tea bags in } 500 \mathrm{~mL} \text { water daily) plus D2 } \\
\text { SC for up to } 6 \text { months. Symptoms disappeared in } 200 \\
\text { patients; } 400 \text { patients improved after having received } \\
\text { additional conventional medication for the first 3-4 weeks. }\end{array}$ & 1983 & $\begin{array}{l}\text { Warning cited in } \\
\text { Schmidt [44] }\end{array}$ \\
\hline Rheumatoid arthritis & $\begin{array}{l}\mathrm{O} \\
1\end{array}$ & $\begin{array}{l}\text { Improvement after treatment with low-potency } \\
\text { Harpagophytum i.v. and orally, duration not provided. }\end{array}$ & 1987 & Stübler [496,497] \\
\hline DJD & $\begin{array}{c}\mathrm{O} \\
553\end{array}$ & $\begin{array}{l}\text { Patients treated with } 2-6 \text { capsules of } 400 \text { mg Harpagophytum } \\
\text { extract }(1.5-2.5: 1) \text { for } 8 \text { to } 180 \text { days. Outcomes confirmed } \\
\text { RCT results in terms of efficacy and safety. }\end{array}$ & 2000 & Müller et al. [498] \\
\hline DJD & $\begin{array}{c}\mathrm{O} \\
255\end{array}$ & $\begin{array}{l}\text { Post-marketing surveillance study of biopsychosocial } \\
\text { determinants and treatment response. Patients treated with } \\
\text { Harpagophytum extract ( } 60 \text { mg harpagoside/day) for } \\
2 \text { months. Outcome parameters were significantly worse in } \\
\text { non-responders. }\end{array}$ & 2009 & $\begin{array}{l}\text { Thanner et al. } \\
\text { [499] }\end{array}$ \\
\hline
\end{tabular}


Table 10. Cont.

\begin{tabular}{|c|c|c|c|c|}
\hline Indication & $\begin{array}{c}\text { Trial Type, } \\
\text { Size }\end{array}$ & Results & Year & Reference \\
\hline $\begin{array}{l}\text { CP, DJD, dyspepsia, } \\
\text { hypercholes- } \\
\text { terolemia, } \\
\text { detoxication }\end{array}$ & $\begin{array}{l}\mathrm{O}, \mathrm{CR} \\
700+\end{array}$ & $\begin{array}{l}\text { Harpagophytum tea, up to } 12 \text { weeks, D2, SC, } 20 \text { injections, } \\
\text { further improvement with additional D2 i.v. and tea. }\end{array}$ & 1978 & Schmidt [130] \\
\hline $\begin{array}{l}\text { Diabetes mellitus } \\
\text { with lipometabolic } \\
\text { disorder }\end{array}$ & $\begin{array}{l}\text { OT } \\
10\end{array}$ & $\begin{array}{l}4 \text { patients } 3 \text { weeks, } 6 \text { patients } 4 \text { and } 3 \text { weeks, over a total of } \\
6 \text { months; Harpagophytum tea, amount not specified; } \\
\text { cholesterol, lipid, and blood sugar levels normalized. }\end{array}$ & 1974 & Hoppe [500] \\
\hline $\begin{array}{l}\text { Hypercholesterolemia } \\
\text { and hyperuricemia }\end{array}$ & $\begin{array}{l}\text { OT } \\
100\end{array}$ & $\begin{array}{l}\text { Harpagophytum tea, } 2 \text { tea bags per } \frac{1}{2} \mathrm{~L} \text { water, } 3 \times \text { daily before } \\
\text { meals } 1 / 3 \text { of the tea; } 20-21 \text { days; lowered cholesterol levels in } \\
80 \% \text {, normal levels in } 45 \%, 66 \% \text { improvement in hyperuricemia. }\end{array}$ & 1978 & Grünewald [405] \\
\hline DJD & $\begin{array}{l}\text { OT } \\
13\end{array}$ & $\begin{array}{l}\text { Harpagophytum extract ( }<30 \mathrm{mg} \text { harpagoside/day), for } \\
6 \text { weeks, followed up for another six weeks; no overall } \\
\text { statistically significant improvements in the conditions. }\end{array}$ & 1981 & $\begin{array}{l}\text { Grahame and } \\
\text { Robinson [501] }\end{array}$ \\
\hline DJD & $\begin{array}{l}\text { OT } \\
630\end{array}$ & $\begin{array}{l}42 \% \text { to } 85 \% \text { of the patients (depending on grouping) showed } \\
\text { improvements after } 6 \text { months with Harpagophytum extract } \\
\text { (>90 mg harpagoside/day). }\end{array}$ & 1982 & Belaiche [502] \\
\hline DJD & $\begin{array}{l}\text { OT } \\
38\end{array}$ & $\begin{array}{l}\text { Comparison of Formica rufa D6 with Harpagophytum D4, for } \\
3 \text { months; improvement in pain severity and mobility with } \\
\text { both, Formica rufa slightly superior. }\end{array}$ & 1991 & Kröner [503] \\
\hline $\begin{array}{l}\text { Effect on eicosanoid } \\
\text { biosynthesis }\end{array}$ & $\begin{array}{l}\text { OT } \\
34(25 / 8) \\
\text { healthy } \\
\text { volunteers }\end{array}$ & $\begin{array}{l}\text { Harpagophytum, } 4 \text { capsules ( } 500 \mathrm{mg} \text { powder, } 3 \% \text { of total } \\
\text { glucoiridoids) daily for } 21 \text { days. No effect vs. control. }\end{array}$ & 1992 & $\begin{array}{l}\text { Moussard et al. } \\
\text { [504] }\end{array}$ \\
\hline MSD & $\begin{array}{c}\text { OT } \\
102(51,51)\end{array}$ & $\begin{array}{l}\text { Patients treated with Harpagophytum extract ( } 30 \mathrm{mg} \\
\text { harpagoside/day) or conventional therapy (mainly oral } \\
\text { NSAIDs). Number of pain-free patients and changes in } \\
\text { Arhus scores after } 4 \text { and } 6 \text { weeks of treatment was } \\
\text { comparable between the groups. }\end{array}$ & 1997 & $\begin{array}{l}\text { Chrubasik et al. } \\
\text { [505] }\end{array}$ \\
\hline DJD & $\begin{array}{l}\text { OT } \\
43\end{array}$ & $\begin{array}{l}\text { Harpagophytum powder } 3 \mathrm{~g} \text { daily for } 60 \text { days. Reduction of } \\
\text { pain intensity in } 89 \% \text {, increased mobility in } 83 \% \text {. }\end{array}$ & 1997 & $\begin{array}{l}\text { Pinget and } \\
\text { Lecomte [506] }\end{array}$ \\
\hline MSD & $\begin{array}{c}\text { OT } \\
2053\end{array}$ & $\begin{array}{l}\text { Patients treated with Harpagophytum extract ( } 30 \mathrm{mg} \\
\text { harpagoside/day) for } 6 \text { weeks. Symptoms improved over time. }\end{array}$ & 1999 & $\begin{array}{l}\text { Schwarz et al. } \\
\text { [507] }\end{array}$ \\
\hline DJD & $\begin{array}{l}\text { OT } \\
45\end{array}$ & $\begin{array}{l}\text { Patients treated with Harpagophytum extract ( } 30 \mathrm{mg} \\
\text { harpagoside/day) for two weeks plus NSAID treatment, and } \\
\text { devil's claw alone, for four weeks. No worsening of scores } \\
\text { was observed during treatment with devil's claw alone. }\end{array}$ & 2000 & $\begin{array}{l}\text { Szczepanski et al. } \\
\text { [508] }\end{array}$ \\
\hline MSD & $\begin{array}{c}\text { OT } \\
1026\end{array}$ & $\begin{array}{l}\text { Patients treated with Harpagophytum extract ( } 30 \mathrm{mg} \\
\text { harpagoside/day) for } 6 \text { weeks. Symptoms improved. }\end{array}$ & 2000 & Usbeck $[509,510]$ \\
\hline MSD & $\begin{array}{l}\text { OT } \\
130\end{array}$ & $\begin{array}{l}\text { Patients treated with Harpagophytum extract ( } \sim 30 \mathrm{mg} \\
\text { harpagoside/day) for } 8 \text { weeks. Arhus back pain index } \\
\text { decreased significantly during treatment. Other measures } \\
\text { also improved significantly. }\end{array}$ & 2001 & $\begin{array}{l}\text { Laudahn et al. } \\
\text { [511-513] }\end{array}$ \\
\hline DJD & $\begin{array}{l}\text { OT } \\
583\end{array}$ & $\begin{array}{l}\text { Patients treated with Harpagophytum extract }(\sim 30 \mathrm{mg} \\
\text { harpagoside/day) for } 8 \text { weeks. Symptoms improved and the } \\
\text { dose of co-medication (NSAIDs) could be reduced. }\end{array}$ & 2001 & Schendel [514] \\
\hline DJD & $\begin{array}{l}\text { OT } \\
675\end{array}$ & $\begin{array}{l}\text { Patients treated with Harpagophytum extract ( } 30 \mathrm{mg} \\
\text { harpagoside/day) for } 8 \text { weeks. Efficacy rated good or very } \\
\text { good in } 82 \% \text { of cases. The symptom scores decreased, and } \\
\text { co-medication was successfully reduced or even discontinued. }\end{array}$ & 2001 & $\begin{array}{l}\text { Ribbat and } \\
\text { Schakau [515] }\end{array}$ \\
\hline
\end{tabular}


Table 10. Cont.

\begin{tabular}{|c|c|c|c|c|}
\hline Indication & $\begin{array}{l}\text { Trial Type, } \\
\text { Size }\end{array}$ & Results & Year & Reference \\
\hline MSD & $\begin{array}{l}\text { OT } \\
250\end{array}$ & $\begin{array}{l}\text { Patients treated with Harpagophytum extract ( } 60 \mathrm{mg} \\
\text { harpagoside/day) for } 8 \text { weeks. Both generic and } \\
\text { disease-specific outcome measures improved. }\end{array}$ & 2002 & $\begin{array}{l}\text { Chrubasik et al. } \\
\qquad[516]\end{array}$ \\
\hline DJD & $\begin{array}{l}\text { OT } \\
614\end{array}$ & $\begin{array}{l}\text { Patients treated with Harpagophytum extract ( } 480 \mathrm{mg} \text { twice } \\
\text { daily) for } 8 \text { weeks. Symptoms improved in the majority of } \\
\text { patients; treatment was well-tolerated. }\end{array}$ & 2003 & $\begin{array}{l}\text { Kloker and } \\
\text { Flammersfeld } \\
{[517,518]}\end{array}$ \\
\hline DJD & $\begin{array}{l}\mathrm{OT} \\
75\end{array}$ & $\begin{array}{l}\text { Patients treated with Harpagophytum extract ( } 50 \mathrm{mg} \\
\text { harpagoside/day) for } 12 \text { weeks. WOMAC index and } 10 \mathrm{~cm} \\
\text { VAS pain scale improved notably. }\end{array}$ & 2003 & $\begin{array}{l}\text { Wegener and } \\
\text { Lüpke }[519,520]\end{array}$ \\
\hline MSD & $\begin{array}{l}\text { OT } \\
99\end{array}$ & $\begin{array}{l}\text { Patients treated with Harpagophytum extract ( } \sim 30 \mathrm{mg} \\
\text { harpagoside/day) for } 6 \text { weeks. Symptoms improved. }\end{array}$ & 2005 & $\begin{array}{l}\text { Rütten and Kuhn } \\
\text { [521] }\end{array}$ \\
\hline MSD & $\begin{array}{c}\text { OT } \\
102 \\
(29 / 22 / 51)\end{array}$ & $\begin{array}{l}\text { Patients treated with Harpagophytum extract ( } ~ 30 \mathrm{mg} \\
\text { harpagoside/day) and/or conventional therapy for } 6 \text { weeks. } \\
\text { Efficacy was found in all groups, advantages for devil's claw } \\
\text { were not statistically significant. }\end{array}$ & 2005 & $\begin{array}{l}\text { Schmidt et al. } \\
\quad[522,523]\end{array}$ \\
\hline DJD & $\begin{array}{l}\mathrm{OT} \\
65\end{array}$ & $\begin{array}{l}\text { Patients treated with combination of Harpagophytum } \\
\text { procumbens, Zingiber officinale, and Urtica sp. (ratio not } \\
\text { disclosed) for } 8 \text { weeks. Improvements in all efficacy } \\
\text { parameters were observed. }\end{array}$ & 2005 & Sohail et al. [524] \\
\hline Endometriosis & $\begin{array}{l}\text { OT } \\
6,12\end{array}$ & $\begin{array}{l}\text { Patients treated with Harpagophytum extract (1600 mg daily) } \\
\text { for } 12 \text { weeks. Reduction of symptoms in } 4 \text { (6) patients after } 4 \\
\text { weeks, in all patients after } 12 \text { weeks. }\end{array}$ & $\begin{array}{l}2005 \\
2006\end{array}$ & $\begin{array}{l}\text { Arndt et al. } \\
{[525,526]}\end{array}$ \\
\hline DJD & $\begin{array}{l}\text { OT } \\
259\end{array}$ & $\begin{array}{l}\text { Patients treated with Harpagophytum extract }(1.5-3: 1,960 \mathrm{mg} \\
\text { daily) and NSAIDs for } 8 \text { weeks. At the end of the treatment, } \\
44.8 \% \text { could decrease NSAID dosage. All parameters } \\
\text { improved significantly. }\end{array}$ & 2006 & $\begin{array}{l}\text { Suter et al. } \\
{[527,528]}\end{array}$ \\
\hline MSD & $\begin{array}{l}\text { OT } \\
114\end{array}$ & $\begin{array}{l}\text { Patients treated with Harpagophytum extract ( } 60 \mathrm{mg} \\
\text { harpagoside/day) for up to } 54 \text { weeks. Most outcome scores } \\
\text { improved significantly over time. }\end{array}$ & 2007 & $\begin{array}{l}\text { Chrubasik et al. } \\
\text { [529] }\end{array}$ \\
\hline DJD & $\begin{array}{l}\mathrm{OT} \\
42\end{array}$ & $\begin{array}{l}\text { Patients treated with combination of Harpagophytum } \\
(1800 \mathrm{mg}), \text { Curcuma longa }(1200 \mathrm{mg}) \text {, and bromelain }(900 \mathrm{mg}) \\
\text { daily, plus conventional therapies for } 2 \text { weeks. Clinically } \\
\text { relevant improvement of joint pain scores in all patients. }\end{array}$ & 2014 & $\begin{array}{l}\text { Conrozier et al. } \\
\text { [530] }\end{array}$ \\
\hline DJD & $\begin{array}{l}\mathrm{OT} \\
20\end{array}$ & $\begin{array}{l}\text { Patients treated with combination of } 500 \mathrm{mg} \text { glucosamine } \\
\text { sulfate, } 400 \mathrm{mg} \text { chondroitin sulfate, } 10 \mathrm{mg} \text { collagen type II, } \\
\text { and } 40 \mathrm{mg} \text { Harpagophytum per day for } 12 \text { months. Femoral } \\
\text { hyaline cartilage thickness significantly improved and } \\
\text { radiographic progression of knee osteoarthritis delayed. }\end{array}$ & 2019 & Vreju et al. [531] \\
\hline MSD & $\begin{array}{c}\text { OT } \\
39 / 40 / 16\end{array}$ & $\begin{array}{l}\text { Otherwise healthy subjects with mild/moderate } \\
\text { neck/shoulder pain related to sport; cream containing a } \\
\text { combination of ingredients, including H. procumbens root } \\
\text { extract + standard treatment, standard treatment, diclofenac } \\
\text { patch + standard treatment respectively, for } 2 \text { weeks; } \\
\text { significant improvement in pain, stiffness, mobility, and } \\
\text { working capacity, compared to non-cream groups. }\end{array}$ & 2021 & Hu et al. [532] \\
\hline
\end{tabular}

$\mathrm{CP}=$ chronic polyarthritis; IA = intra-articular; $\mathrm{SC}=$ subcutaneous; DJD = degenerative joint diseases (osteoarthritis); MSD = musculoskeletal disorders (low back pain); OT = observational trial; $\mathrm{O}=$ observation; $\mathrm{CR}=$ case report; NSAID = non-steroidal anti-inflammatory drug; WOMAC = Western Ontario and McMaster Universities. 
Table 11. RCTs conducted between 1980 and 2017.

\begin{tabular}{|c|c|c|c|c|}
\hline DJD & $\begin{array}{c}\text { RCT } \\
39\end{array}$ & $\begin{array}{l}400 \mathrm{mg} \text { Harpagophytum extract (not specified), and } 25 \mathrm{mg} \\
\text { diclofenac, or placebo } 3 \times \text { daily for } 6 \text { months. Overall confirmation } \\
\text { of anti-inflammatory effects without side effects. }\end{array}$ & $\sim 1980$ & $\begin{array}{c}\text { Chaouat, cited in } \\
{[66,67]}\end{array}$ \\
\hline DJD & $\begin{array}{c}\text { RCT } \\
50(25 / 25)\end{array}$ & $\begin{array}{l}\text { Harpagophytum extract ( }<30 \mathrm{mg} \text { harpagoside/day) and } \\
\text { phenybutazone ( } 300 \mathrm{mg} \text { per day for the first four days, then } \\
200 \mathrm{mg} \text { ) respectively, for } 28 \text { days. Devil's claw found equally } \\
\text { effective to phenybutazone. }\end{array}$ & 1980 & Schrüffler [533] \\
\hline DJD & $\begin{array}{c}\text { RCT } \\
50(25 / 25)\end{array}$ & $\begin{array}{l}\text { Patients treated with Harpagophytum extract }(<20 \mathrm{mg} \\
\text { harpagoside/day) or placebo for three weeks showed a significant } \\
\text { decrease in pain severity vs. placebo. }\end{array}$ & 1984 & Guyader [534] \\
\hline DJD & $\begin{array}{c}\text { RCT } \\
100(50 / 50)\end{array}$ & $\begin{array}{l}\text { Patients treated with Harpagophytum extract ( } 60 \mathrm{mg} \text { harpagoside/day) } \\
\text { or placebo for } 30 \text { days. Only } 6 \text { patients in the verum group still } \\
\text { experienced moderate pain vs. } 32 \text { in the placebo group. }\end{array}$ & 1990 & $\begin{array}{l}\text { Pinget and Lecomte } \\
\text { [535] }\end{array}$ \\
\hline DJD & $\begin{array}{c}\text { RCT } \\
89(45 / 44)\end{array}$ & $\begin{array}{l}\text { Patients treated with Harpagophytum extract }(60 \mathrm{mg} \\
\text { harpagoside/day) or placebo for two months. Significant decrease } \\
\text { in severity of pain and significant increase in spinal and } \\
\text { cofexomoral mobility vs. placebo. }\end{array}$ & 1992 & $\begin{array}{l}\text { Lecomte and Costa } \\
\text { [536] }\end{array}$ \\
\hline MSD & $\begin{array}{c}\mathrm{RCT} \\
118(59,59)\end{array}$ & $\begin{array}{l}\text { Patients treated with Harpagophytum extract ( } 50 \mathrm{mg} \\
\text { harpagoside/day) or placebo for } 4 \text { weeks. Treatment group used } \\
\text { less analgesics, had greater improvement in median Arhus scores } \\
(20 \% \text { vs. } 8 \% ; p<0.059) \text {, and had more patients pain-free at the end } \\
(9 / 51 \text { vs. } 1 / 54 ; p=0.008) \text {. }\end{array}$ & 1996 & $\begin{array}{l}\text { Chrubasik et al. } \\
\text { [537-539] }\end{array}$ \\
\hline MSD & $\begin{array}{c}\text { RCT } \\
109(54 / 55)\end{array}$ & $\begin{array}{l}\text { Patients treated with Harpagophytum extract }(50 \mathrm{mg} \\
\text { harpagoside/day) or placebo for } 4 \text { weeks. Rescue medication: } \\
\text { tramadol. Significant improvement in Arhus index and pain } \\
\text { index, and co-medication reduced vs. placebo. }\end{array}$ & 1997 & Chrubasik et al. [540] \\
\hline DJD & $\begin{array}{c}\text { RCT } \\
100(50 / 50)\end{array}$ & $\begin{array}{l}\text { Patients treated with Harpagophytum extract }(30 \mathrm{mg} \\
\text { harpagoside/day) or placebo for } 30 \text { days. Favorable effects were } \\
\text { evident after } 10 \text { days vs. placebo. }\end{array}$ & 1997 & $\begin{array}{c}\text { Schmelz and } \\
\text { Hämmerle [541] }\end{array}$ \\
\hline MSD & $\begin{array}{c}\text { RCT } \\
197(65 / 66 / 66)\end{array}$ & $\begin{array}{l}\text { Patients treated with Harpagophytum extract ( } 50 \mathrm{mg}(1), 100 \mathrm{mg}(2) \\
\text { harpagoside/day) or placebo ( } 3 \text { ) for four weeks. 6, 10, and } 3 \\
\text { patients were pain-free in groups } 1,2 \text { and } 3 \text {, respectively. Arhus } \\
\text { index score decreased but not statistically significant. Dose-related } \\
\text { effect not confirmed. }\end{array}$ & 1999 & Chrubasik et al. [542] \\
\hline DJD & $\begin{array}{c}\mathrm{RCT} \\
122(62 / 60)\end{array}$ & $\begin{array}{l}\text { Patients treated with Harpagophytum extract ( } 57 \mathrm{mg} \\
\text { harpagoside/day) or diacerhein at } 100 \mathrm{mg} \text { daily for four months. } \\
\text { Results showed significant improvement in both groups at a } \\
\text { similar rate. }\end{array}$ & 2000 & $\begin{array}{c}\text { Chantre et al. } \\
{[543,544]}\end{array}$ \\
\hline MSD & $\begin{array}{c}\text { RCT } \\
63(31 / 32)\end{array}$ & $\begin{array}{l}\text { Patients treated with Harpagophytum extract ( } 30 \mathrm{mg} \\
\text { harpagoside/day) or placebo for } 4 \text { weeks. Significant efficacy for } \\
\text { visual analogue scale, pressure algometer test, muscle stiffness } \\
\text { test, and muscular ischemia test. No differences to placebo in } \\
\text { anti-nociceptive muscular reflexes or electromyogram activity. }\end{array}$ & 2000 & $\begin{array}{c}\text { Göbel et al. } \\
{[512,513,545,546]}\end{array}$ \\
\hline DJD & $\begin{array}{c}\mathrm{RCT} \\
46(24 / 22)\end{array}$ & $\begin{array}{l}\text { Patients treated with ibuprofen }(800 \mathrm{mg}) \text { and Harpagophytum } \\
\text { extract ( } 30 \mathrm{mg} \text { harpagoside/day) or placebo for } 20 \text { weeks. } \\
\text { WOMAC scores decreased similarly, but during an ibuprofen-free } \\
\text { period, symptoms worsened less than } 20 \% \text { for } 71 \% \text { of devil's claw } \\
\text { patients vs. } 41 \% \text { of placebo patients. }\end{array}$ & 2001 & Frerick et al. [547] \\
\hline DJD & $\begin{array}{c}\text { RCT } \\
78(39 / 39)\end{array}$ & $\begin{array}{l}\text { Patients treated with Harpagophytum extract ( } \sim 30 \mathrm{mg} \\
\text { harpagoside/day) or placebo for } 20 \text { weeks. Co-medication } \\
\text { ibuprofen. Symptoms improved similarly for both groups. }\end{array}$ & 2002 & Biller [548] \\
\hline MSD & $\begin{array}{c}\text { RCT } \\
88(44 / 44)\end{array}$ & $\begin{array}{l}\text { Patients treated with Harpagophytum extract ( } 60 \mathrm{mg} \\
\text { harpagoside/day) for } 6 \text { weeks or } 12.5 \mathrm{mg} \text { /day of rofecoxib. } \\
\text { Outcome scores improved similarly for both groups. Follow-up } \\
\text { confirmed the results of the pilot study. }\end{array}$ & 2003 & $\begin{array}{l}\text { Chrubasik et al. } \\
{[538,539,549-552]}\end{array}$ \\
\hline
\end{tabular}


Table 11. Cont.

\begin{tabular}{|c|c|c|c|c|}
\hline MSD & $\begin{array}{c}\text { RCT } \\
97(36 / 31 / 30)\end{array}$ & $\begin{array}{l}\text { Patients treated with Harpagophytum extract ( } \sim 30 \mathrm{mg} \\
\text { harpagoside/day) or NSAID (Voltaren } 150 \mathrm{mg} \text { or Vioxx } 12.5 \mathrm{mg} \text { ), } \\
\text { duration not provided; outcomes show equality of treatment. }\end{array}$ & 2005 & $\begin{array}{c}\text { Lienert et al. } \\
{[553,554]}\end{array}$ \\
\hline DJD & $\begin{array}{c}\text { RCT } \\
60(30 / 30)\end{array}$ & $\begin{array}{l}\text { Patients treated with combination of Harpagophytum and Apium } \\
\text { graveolens extract (cream, } 1.5 \mathrm{~cm} \text {, twice daily) or placebo for } 2 \\
\text { weeks. Treatment group showed significant improvement in } \\
\text { algometer, flexion, and extension readings. }\end{array}$ & 2006 & Pillay [555] \\
\hline $\begin{array}{l}\text { Sore } \\
\text { throat } \\
\text { after } \\
\text { tra- } \\
\text { cheal } \\
\text { intuba- } \\
\text { tion }\end{array}$ & $\begin{array}{c}\text { RCT } \\
60(30 / 30)\end{array}$ & $\begin{array}{l}\text { Patients treated with Harpagophytum extract ( } 480 \mathrm{mg} \text { one hour } \\
\text { before intubation) or placebo plus premedication (fentanyl, } \\
\text { midazolam, propofol). No significant difference was observed } \\
\text { between groups. }\end{array}$ & 2016 & Anvari et al. [556] \\
\hline DJD & $\begin{array}{c}\text { RCT } \\
92(46 / 46)\end{array}$ & $\begin{array}{l}\text { Patients treated with combination of Rosa canina, Urtica sp., } \\
\text { Harpagophytum procumbens, and vitamin D ( } 20.0 \text { g puree and } 4.0 \mathrm{~g} \\
\text { juice concentrate, } 160 \mathrm{mg} \text { dry extract, } 108 \mathrm{mg} \text { dry extract, } 5 \mu \mathrm{g} \text {, } \\
\text { respectively) or placebo for } 12 \text { weeks. WOMAC and quality of life } \\
\text { scores significantly improved vs. placebo. }\end{array}$ & 2017 & Moré et al. [557] \\
\hline
\end{tabular}

DJD = degenerative joint diseases (osteoarthritis); MSD = musculo-skeletal disorders (low back pain); RCT = randomized controlled trial; NSAID = non-steroidal anti-inflammatory drug; WOMAC = Western Ontario and McMaster Universities.

\subsection{Safety}

A broad spectrum of claims regarding the safety of Harpagophytum in clinical practice can be found in the literature, ranging from unsubstantiated cautioning against its use altogether $[215,558]$ to overly optimistic perspectives in the lay press. The truth, as it often does, lies somewhere in between.

\subsubsection{Clinical Safety}

Short- and long-term use (on average 30-60 days, in several long-term studies up to 54 weeks) have been described as safe and well-tolerated, and the most reported adverse events in clinical investigations were of mild gastrointestinal nature [559]. These may be related to its anticholinesterase effect in vitro [318,366]. A review of the safety of Harpagophytum preparations [560] concluded that they are likely to be safe with only few and no serious adverse events observed, however, it was also established that further, more rigorous safety investigations are required [561], especially considering that the dosage in most studies was found at the lower limit, and for the recommended long-term use.

\subsubsection{Interaction Potential}

Harpagophytum was found to be a weak inhibitor of CYP 1A2 and CYP 2D6, and a moderate inhibitor of CYP 2C8, CYP 2C9, CYP 2C19, and CYP 3A4 in vitro [397,399,400,562], however, clinical relevance is unlikely [402]. Increased anticoagulant effects have been reported with concurrent anticoagulant use [563-567]. While an interaction is possible, evidence is inconclusive [568] and has only been demonstrated in vitro. Herb-drug interactions and interference with anticoagulants are hypothetical and have not been conclusively demonstrated.

\subsubsection{Adverse Event Reports}

A case of hyponatremia in a patient with systemic hypertension has been associated with Harpagophytum (co-medications were losartan, clonidine, omeprazole, and simvastatin) [569]. Another case report suggests development of grade 2 symptomatic hypertension in a normotensive woman during self-administration of Harpagophytum [570]. However, available data do not suggest interaction potential with conventional antihypertensives at recommended doses (animal studies demonstrating a hypotensive effect used 
much higher doses). A case-controlled surveillance study has associated Harpagophytum with a pancreatoxic potential [571]. One early case report points at a potential allergic reaction after professional exposure to Harpagophytum [572]. Rahman and colleagues [573] included Harpagophytum in a review of botanicals with drug-interaction potential in the elderly with inflammatory bowel disease, however, did not present any causality that would justify concern.

\subsubsection{Side Effects}

Considering the size of the total patient collective from all clinical investigations listed in Section 11.1. (>11,000), and the most common side effects being mild gastrointestinal complaints (nausea, abdominal pain, diarrhea), CNS disorders (dizziness, headache), and allergic skin reactions, the aforementioned case reports should be further investigated, but, until corroborated by new data, their clinical relevance can be deemed as limited.

\subsubsection{Pregnancy and Lactation}

In Vitro data suggest spasmogenic and uterotonic effects in mammalian uterine muscles [412,413]. In the absence of adequate in vivo data [408,409], use during pregnancy and lactation should be cautioned.

\section{Veterinary Applications}

Veterinary applications of devil's claw have received increased attention and gained popularity over the last 15 years, with focus on equines and canines. Colas and colleagues $[250,255,256,574]$ provided methods for detection and control of iridoid glucosides from Harpagophytum in horse urine. Torfs et al. [575] discussed the potential benefits of devil's claw products in veterinary practice and cited one study conducted by Montavon [576] in which ten horses with tarsal osteoarthritis were treated with an herbal powder mix containing Harpagophytum (20 g total) and smaller quantities of Ribes nigrum, Equisetum arvense, and Salix alba for 10 days a month over three consecutive months. The control group received $2 \mathrm{~g}$ of phenylbutazone daily. Locomotor scores improved significantly with the test medication vs. conventional NSAID. However, study results are of limited reliability due to size, lack of blinding, and subjective assessment. Axmann and colleagues $[577,578]$ investigated pharmacokinetics and clinical efficacy of a Harpagophytum extract in horses. They provided a method with which they were able to detect harpagoside in plasma for up to $9 \mathrm{~h}$ after administration. Efficacy was investigated in a RCT design with 40 horses (20/20), the study medication was $10 \mathrm{~g}$ daily of an aqueous Harpagophytum extract (25.3\% harpagoside) or placebo for 8 weeks, and a follow-up after 16 weeks. Locomotor abnormalities were assessed on a treadmill with an optoelectronic motion capture system, and follow-up was conducted via questionnaires. While the objective motion assessment did not yield significant differences between baseline and the end of the study, evaluation of the questionnaires reflected significant improvements and a "lingering" effect in the subjective assessment.

Moreau and colleagues [579] investigated the efficacy of Harpagophytum (harpagoside $>2.7 \%$ ) as part of a complex mixture of ingredients for improving symptoms of canine osteoarthritis in a RCT with 32 dogs (16 per group) over 8 weeks. The primary endpoint, peak vertical force, was significantly higher in treated dogs vs. placebo after 4 and 8 weeks, and clinical signs overall improved with treatment.

Ethnoveterinary uses of devil's claw have also been recorded. Moreki [113] reports on ethnoveterinary practices in Botswana to include the use of a decoction of Harpagophytum in poultry.

A reliable body of clinical data confirming the efficacy of Harpagophytum in veterinary applications is clearly lacking but is needed to better exploit the potential benefits. In this context, it must be noted that the use of devil's claw-just like other analgesics-is highly restricted in equestrian sport. Harpagoside is included in the "Equine Prohibited Substance List" of the Federation Equestre Internationale as a "controlled medication", the 
use of which is prohibited during training and competitions. Curiously, harpagoside is not included in the very same organization's "List of Detection Times", leaving horse owners in the dark as to when to discontinue use prior to a tournament. This lack of clarity may further hamper more prolific use in veterinary practice.

\section{Patents}

As mentioned in Section 10, the majority of patents refer to processing methods, specifically extraction and dosage forms, which constitute the only legitimately patentable intellectual property for the pharmaceutical industry, except in cases where new effects or combinations, not previously described in ethnobotanical use accounts, were elucidated. It is noteworthy that most of the earlier patents listed below in Table 12 (pre-2000) have expired or been withdrawn. Pending patents have been excluded.

Table 12. Patents pertaining to Harpagophytum and its preparations.

\begin{tabular}{|c|c|c|}
\hline Title & Date & Number \\
\hline Food supplement & $4 / 3 / 1984$ & US19810287235 \\
\hline Therapeutically active mixture & $11 / 8 / 1984$ & DE19833316726 \\
\hline Homeopathic remedy for the treatment of rheumatic disorders & $11 / 19 / 1987$ & DE19863616054 \\
\hline Plant-based medicinal composition for internal use & $4 / 22 / 1988$ & FR19860014608 \\
\hline $\begin{array}{l}\text { Medicinal combination based on plants and trace elements for the treatment of rheumatism and } \\
\text { inflammatory states }\end{array}$ & $11 / 10 / 1988$ & FR19870006450 \\
\hline Process for the preparation, by extracting, of Harpagophytum & $7 / 13 / 1992$ & KR19890016112 \\
\hline Anti-pruritic cosmetic composition containing Harpagophytum root extract & $1 / 27 / 1993$ & EP19920402100 \\
\hline Preparation of concentrated plant extract, particularly from Harpagophytum procumbens & $8 / 7 / 1997$ & DE1996103788 \\
\hline $\begin{array}{l}\text { Harpagosid-angereicherter Extrakt aus Harpagophytum procumbens und Verfahren zu seiner } \\
\text { Herstellung [harpagoside- enriched extract of } \boldsymbol{H} \text {. procumbens and its manufacture] }\end{array}$ & 10/2/1997 & DE1996151290 \\
\hline $\begin{array}{l}\text { A purified extract from Harpagophytum procumbens and/or Harpagophytum zeyheri, a process for } \\
\text { its preparation and its use }\end{array}$ & $12 / 18 / 1997$ & Google \\
\hline $\begin{array}{l}\text { Skin care composition contains peroxidized fatty substance, e.g., unsaturated vegetable oils and } \\
\text { plant extract }\end{array}$ & $3 / 20 / 1998$ & FR19960011438 \\
\hline Natural composition for treating bone or joint inflammation & $11 / 26 / 1998$ & WO1998US10758 \\
\hline $\begin{array}{l}\text { Micro-nutritional compositions having a therapeutic effect containing polyunsaturated fatty } \\
\text { acids, trace elements, and vitamins }\end{array}$ & $7 / 16 / 1999$ & FR19980000331 \\
\hline $\begin{array}{l}\text { A method of producing high anti-inflammatory activity extracts from } \\
\text { Harpagophytum procumbens }\end{array}$ & 10/6/1999 & GB19980006971 \\
\hline Effervescent preparation containing a plant extract & $6 / 16 / 1999$ & EP0922450A1 \\
\hline Method for producing high activity extracts from Harpagophytum procumbens & $3 / 6 / 2001$ & US19990280499 \\
\hline $\begin{array}{l}\text { Harpagoside-enriched extract from Harpagophytum procumbens and processes for producing } \\
\text { the same }\end{array}$ & $8 / 28 / 2001$ & US19990155043 \\
\hline Dietary supplement & $12 / 18 / 2001$ & JP20000172296 \\
\hline $\begin{array}{l}\text { Pharmaceutical preparation containing Cibotii rhizoma and Harpagophytum procumbens DC } \\
\text { extracts as main ingredients }\end{array}$ & $6 / 3 / 2002$ & KR20000071397 \\
\hline Skin care preparation & $6 / 4 / 2002$ & JP20000402968 \\
\hline Pharmaceutical composition with anti-atherosclerotic activity & $6 / 5 / 2002$ & EP20010128629 \\
\hline $\begin{array}{l}\text { Use of harpagide-related compound as prophylactic and therapeutic agent of osteoporosis, } \\
\text { arthritis, and disc and pharmaceutical composition containing compound as } \\
\text { effective ingredient }\end{array}$ & $11 / 16 / 2002$ & KR20000071497 \\
\hline $\begin{array}{l}\text { Composition useful for treating or preventing osteoarthritis, especially in horses, containing } \\
\text { extract(s) of Equisetum arvense, Symphytum officinale, and/or Harpagophytum procumbens }\end{array}$ & $3 / 27 / 2003$ & DE2001143146 \\
\hline
\end{tabular}


Table 12. Cont.

\begin{tabular}{|c|c|c|}
\hline Title & Date & Number \\
\hline $\begin{array}{l}\text { Use of active substance mixtures containing tocopherols and Harpagophytum procumbens } \\
\text { extracts for the preparation of a drug against rheumatic arthritis }\end{array}$ & $12 / 17 / 2003$ & EP20020012765 \\
\hline Chewing gum composition with vegetal additives & $7 / 29 / 2004$ & WO2003EP14600 \\
\hline $\begin{array}{l}\text { Pain-relieving agent containing extract of Harpagophytum procumbens, Corydalis turtschanovii, } \\
\text { and Atractylodes japonica }\end{array}$ & $2 / 5 / 2005$ & KR20030052489 \\
\hline $\begin{array}{l}\text { Treating or preventing renal diseases, dysfunction, and/or damage, e.g., degenerative and/or } \\
\text { inflammatory renal disease, using Harpagophytum extract or harpagoside }\end{array}$ & $3 / 10 / 2005$ & DE2003126556 \\
\hline Phyto-composition for the treatment of articular diseases & & WO2005092355 \\
\hline Use of devil's claw (Harpagophytum procumbens) root extracts for endometriosis treatment & $11 / 2 / 2006$ & WO2006EP61831 \\
\hline A method for separating harpagide from Harpagophytum procumbens & $2 / 5 / 2007$ & KR20050102609 \\
\hline Activator of peroxisome proliferator-activated receptor (PPAR) & $5 / 17 / 2007$ & JP20050317156 \\
\hline Adjuvant composition for physiotherapy & $7 / 24 / 2007$ & KR20060005183 \\
\hline Maillard reaction inhibitor, skin care preparation containing the same, and food and beverage & $10 / 4 / 2007$ & JP20060080104 \\
\hline Phyto-composition for the treatment of joint diseases & $12 / 13 / 2007$ & US20050594439 \\
\hline Natural remedy-dietary supplement combination product & $9 / 4 / 2008$ & US20060815432 \\
\hline Root extract of Harpagophytum for stimulating hair growth & $5 / 27 / 2009$ & EP20070802633 \\
\hline Skin care preparation, oral composition, and food and drink & $10 / 22 / 2009$ & JP20080091677 \\
\hline Novel method for preparing purified extracts of Harpagophytum procumbens & $12 / 9 / 2010$ & US20080599146 \\
\hline Animal food compositions & $7 / 21 / 2011$ & WO2010US60804 \\
\hline Compositions comprising plant extracts and use thereof for treating inflammation & $10 / 27 / 2011$ & US200913120739 \\
\hline Anti-inflammatory composition & $12 / 21 / 2011$ & EP20110170436 \\
\hline Antirheumatic body cream composition & $12 / 30 / 2011$ & RO20110000644 \\
\hline $\begin{array}{l}\text { Pharmaceutical composition for preventing and treating metabolic bone disease comprising } \\
\text { of Harpagophytum }\end{array}$ & $6 / 18 / 2012$ & KR20110147135 \\
\hline
\end{tabular}

Phyto-concentrated composition, useful as antispasmodic relaxant, and muscular comfort to, e.g., enhance relaxation of painfully contracted muscle tissue, comprises, e.g., cannabis sativa and an excipient comprising, e.g., castor oil

Nonabrasive toothpaste containing enzyme papain, Harpagophytum extract d,l-pyrrolidone carboxylate n-cocoyl ethyl arginate, and sodium fluoride

10/12/2012 FR20110001030

7/20/2013 RU20120101119

Cosmetic composition for calming and applying an electric current of skins and manufacturing the same

Anti-rheumatism medicinal liquor and preparation method thereof

12/27/2013 KR20120065152

Composition containing chondroitin sulfate and hyaluronidase

3/19/2014 CN20131645408

Mucoadhesive devil's claw extracts (Harpagophytum procumbens) and uses thereof

12/10/2014 RU20130123301

Compositions for alleviating, preventing, or treating pain comprising Harpagophytum procumbens and Acanthopanax senticosus extracts as active ingredients

Traditional Chinese medicine composite for treating gout

3/11/2015 EP20140184267

Cell line cultures from plants belonging to the Harpagophytum genus

6/8/2015 KR20130146128

Method for preparing purified extracts of Harpagophytum procumbens

7/8/2015 CN20151209743

Oral herbal pain killer formulations

1/4/2018 WO2017EP65814

30/10/2018 US20100311675A1

Polyherbal transdermal patch for pain management and its process of preparation

15/10/2020 WO2020208395A1

External medicine for inhibiting postoperative venous thrombosis and application thereof

22/10/2020 WO2020212820A2

Freedom (nutritional supplement)

19/2/2021 CN109589331B

$9 / 2 / 2021$

US20200060320A1 


\section{Discussion and Conclusions}

Devil's claw is a well-established phytopharmaceutical. A large body of data exists in which composition, pharmacological activities, and clinical effects are elucidated, and in turn support and affirm traditional use applications. Nonetheless, several aspects requiring further investigation were highlighted by this review.

Revision of the genus to account for introgression, geographical, and biochemical variation, and geo-authenticity is needed.

In view of the interchangeable use of both Harpagophytum species and mixtures thereof in clinical practice, further comparative examination of the composition of both species is needed. Verbascoside as an anti-inflammatory compound present in Harpagophytum could be an interesting target of future research.

Despite some inconsistent outcomes and contradictory results, pharmacological evidence appears to be overall sufficient to support clinical use. Sufficient pharmacological differentiation between Harpagophytum species, however, is lacking.

Toxicological evaluations of Harpagophytum indicate a low toxicity in animal models. While genotoxicity testing is part of the regulatory requirements for the market authorization of herbal medicinal products in Europe, results are proprietary (product-related) and have not been published. Adequate tests on reproductive toxicity, genotoxicity, and carcinogenicity, performed according to currently valid OECD guidelines, need to be made publicly available.

While there may be strong clinical evidence that devil's claw preparations are effective in the treatment of degenerative joint diseases and musculoskeletal disorders in principle, this conclusion cannot be extended to specific preparations, because of the varying pharmaceutical quality of individual preparations.

Further investigations are required (a) to identify the therapeutically active substances or fractions and thus enable tests which (b) use accordingly standardized and sufficiently dosed preparations with a carefully designed setup and methodology in order to obtain quantifiable results for the efficacy of devil's claw preparations. These need to be conducted with both Harpagophytum spp. individually but prepared identically. Trial designs should be guided by the recommendations of the International Council for Harmonization of Technical Requirements for Pharmaceuticals for Human Use (ICH). Specifically, both species could be compared in a two-arm cross-over design. Conventional medication could be added as a third arm to assess comparative efficacy. Studies should be of adequate power, randomized, placebo-controlled, and double-blinded. Problematic in an ethical sense is the denial of "first aid" medication in placebo-controlled studies, permission of which would confound outcomes. Outcomes should be objective or at least a combination of objective and subjective measures.

Further research is also warranted in the area of clinical safety, specifically with regard to the drug interaction potential of devil's claw preparations. Until then, safety considerations as expressed in current compendia, e.g., [15], should be considered appropriate.

Funding: This research received no external funding.

Institutional Review Board Statement: Not applicable.

Informed Consent Statement: Not applicable.

Data Availability Statement: All data has been presented in the main text.

Acknowledgments: Ernst Schneider, Mathias Schmidt, Sigrun Chrubasik, Margret Moré, Dave Cole, Josef Brinckmann, Wolfram Hartmann, Cyril Lombard, Ben-Erik van Wyk, and Karen Nott kindly assisted with the procurement of some illusive publications.

Conflicts of Interest: The author declares no conflict of interest. 


\section{References}

1. Ihlenfeldt, H.-D.; Hartmann, H. Die Gattung Harpagophytum (Burch.) DC. Ex Meissn. (Monographie der afrikanischen Pedaliaceae II). Hambg. Staatsinst. Allg. Bot. Mitt. 1970, 13, 15-69.

2. Muzila, M.; Werlemark, G.; Ortiz, R.; Sehic, J.; Fatih, M.; Setshogo, M.; Mpoloka, W.; Nybom, H. Assessment of diversity in Harpagophytum with RAPD and ISSR markers provides evidence of introgression. Hereditas 2014, 151, 91-101. [CrossRef]

3. Brendler, T.; van Wyk, B.E. A historical, scientific and commercial perspective on the medicinal use of Pelargonium sidoides (Geraniaceae). J. Ethnopharmacol. 2008, 119, 420-433. [CrossRef]

4. Stander, M.A.; Brendler, T.; Redelinghuys, H.; Van Wyk, B.E. The commercial history of Cape herbal teas and the analysis of phenolic compounds in historic teas from a depository of 1933. J. Food Compos. Anal. 2019, 76, 66-73. [CrossRef]

5. Low, C.H. Different histories of buchu: Euro-American appropriation of San and Khoekhoe knowledge of buchu plants. Environ. Hist. 2007, 13, 333-361. [CrossRef]

6. Brendler, T.; Cock, I.E. A short history of Cape aloe bitters. S. Afr. J. Bot. 2021. under review.

7. Helmstädter, A. Xysmalobium undulatum (Uzara) research-How everything began. J. Ethnopharmacol. 2015, 164, 385-387. [CrossRef] [PubMed]

8. Brendler, T. The rise and fall of Hoodia: A lesson on the art and science of natural product commercialization. In African Natural Plant Products, Volume III: Discoveries and Innovations in Chemistry, Bioactivity, and Applications; ACS Publications: Washington, DC, USA, 2020; pp. 313-324, ISBN 1947-5918.

9. Van Wyk, B.E. A review of commercially important African medicinal plants. J. Ethnopharmacol. 2015, 176, 118-134. [CrossRef]

10. CITES. Inclusion of Harpagophytum Procumbens in Appendix II in Accordance with Article II 2(a) and Inclusion of Harpagophytum Zeyheri in Appendix II in Accordance with Article II 2(b) for Reasons of Look-Alike Problems. 2000, pp. 1-9. Available online: https:/ / cites.org/sites/default/files/eng/cop/11/prop/60.pdf (accessed on 12 April 2021).

11. Czygan, F.-C.; Krüger, A.; Schier, W.; Volk, O.H. Pharmazeutisch-biologische Untersuchungen der Gattung Harpagophytum (Bruch.) DC ex Meissn. 1. Mitteilung: Phytochemische Standardisierung von Tubera Harpagophyti. Dtsch. Apoth. Ztg. 1977, 117, 1431-1434.

12. Eich, J.; Schmidt, M.; Betti, G.J.R. HPLC analysis of iridoid compounds of Harpagophytum taxa: Quality control of pharmaceutical drug material. Pharm. Pharmacol. Lett. 1998, 8, 75-78.

13. Feistel, B.; Gaedcke, F. Analytical identification of Radix Harpagophyti procumbentis and zeyheri. Z. Phytother. 2000, 21, $246-251$.

14. Nott, K. A Survey of the Harvesting and Export of Harpagophytum procumbens and Harpagophytum Zeyheri in SWA/Namibia; Etosha Ecological Institute: Okaukuejo, Namibia, 1986.

15. EMA. European Union Herbal Monograph on Harpagophytum procumbens DC. and/or Harpagophytum zeyheri Decne., Radix. EMA/HMPC/627057/2015; Committee on Herbal Medicinal Products (HMPC): London, UK, 2016.

16. European Scientific Cooperative on Phytotherapy. Harpagophyti radix. In ESCOP Monographs, 2nd ed.; Thieme: Stuttgart, Germany, 2003; pp. 233-240.

17. Menghini, L.; Recinella, L.; Leone, S.; Chiavaroli, A.; Cicala, C.; Brunetti, L.; Vladimir-Knezevic, S.; Orlando, G.; Ferrante, C. Devil's claw (Harpagophytum procumbens) and chronic inflammatory diseases: A concise overview on preclinical and clinical data. Phytother. Res. 2019, 33, 2152-2162. [CrossRef] [PubMed]

18. Stewart, K.M.; Cole, D. The commercial harvest of devil's claw (Harpagophytum spp.) in southern Africa: The devil's in the details. J. Ethnopharmacol. 2005, 100, 225-236. [CrossRef]

19. Baghdikian, B.; Lanhers, M.C.; Fleurentin, J.; Ollivier, E.; Maillard, C.; Balansard, G.; Mortier, F. An analytical study, anti-inflammatory and analgesic effects of Harpagophytum procumbens and Harpagophytum zeyheri. Planta Med. 1997, 63, 171-176. [CrossRef]

20. Anonymous. Harpagophytum procumbens (devil's claw). Altern. Med. Rev. 2008, 13, 248-252.

21. Barnes, J. Charms \& harms: Devil's claw. J. Prim. Health Care 2009, 1, 238-239. [CrossRef]

22. Caprasse, M. Description, identification et usages thérapeutiques de la «griffe du diable»: Harpagophytum procumbens DC. J. Pharm. Belg. 1980, 35, 143-149.

23. Chrubasik, S. Wirksamkeit pflanzlicher Schmerzmittel am Beispiel des Teufelskrallenwurzelextrakts. Orthopäde 2004, 33, 804-808. [CrossRef] [PubMed]

24. Czygan, F.-C. Harpago- oder Teufelskrallentee, das Auf und Ab einer Modedroge. Z. Phytother. 1984, 5, $922-925$.

25. Czygan, F.-C. Nochmals Harpagophytum. Z. Phytother. 1984, 5, 972.

26. Czygan, F.-C. Portrait einer Arzneipflanze: Harpagophytum-Teufelskralle. Z. Phytother. 1987, 8, 17-20.

27. Dittrich, C. Harpagophytum procumbens DC. Österr. Apoth. Ztg. 1974, 28, 53-54.

28. Esdorn, I. Afrikanische Reiseeindrücke in pharmazeutischer und kultureller Hinsicht. Dtsch. Apoth. Ztg. 1963, 103, 785-789.

29. Faivre, C.; Ghedira, K.; Goetz, P.; Lejeune, R. Harpagophytum procumbens (Pedaliaceae). Phytothérapie 2007, 5, 150-153. [CrossRef]

30. Georgiev, M.; Ivanovska, N.; Alipieva, K.; Dimitrova, P.; Verpoorte, R. Harpagoside: From Kalahari Desert to pharmacy shelf. Phytochemistry 2013, 92, 8-15. [CrossRef]

31. Hansen, C. Arzneistoff Porträt-Die Afrikanische Teufelskralle-Voodoo oder wirksames Arzneimittel? Dtsch. Apoth. Ztg. 2000, $140,85-89$.

32. Jaspersen-Schib, R. Harpagophyti radix-Wirklich eine Wunderdroge. Dtsch. Apoth. Ztg. 1990, $130,71$.

33. Kampffmeyer, H. Teufelskralle-Gibt es eine therapeutische Wirkung? ZFA 1980, 56, 618.

34. Kannacher, M. Harpagophytum procumbens_-Die Teufelskralle. Tubera harpagophyti, die Speicherknollen. Volksheilkunde 1993, $45,44$. 
35. Lis, K. Diabelska moc czarciego pazura. Reumatologia 2010, 48, 128-132.

36. McGregor, G.; Fiebich, B.; Wartenberg, A.; Brien, S.; Lewith, G.; Wegener, T. Devil's claw (Harpagophytum procumbens): An anti-inflammatory herb with therapeutic potential. Phytochem. Rev. 2005, 4, 47-53. [CrossRef]

37. McGregor, G.P. Harpagophytum procumbens-Traditional anti-inflammatory herbal drug with broad therapeutic potential. In Herbal Drugs: Ethnomedicine to Modern Medicine; Springer: New York, NY, USA, 2009; pp. 81-95.

38. Miraldi, E.; Biagi, M.; Giachetti, D. A comprehensive systematic pharmacological review on Harpagophytum procumbens DC. (Devil's claw). Biol. Sci. PJSIR 2008, 51, 165-176.

39. Mncwangi, N.; Chen, W.; Vermaak, I.; Viljoen, A.; Gericke, N. Devil's claw-A review of the ethnobotany, phytochemistry and biological activity of Harpagophytum procumbens. J. Ethnopharmacol. 2012, 143, 755-771. [CrossRef] [PubMed]

40. Olivier, D.K. The Ethnobotany and Chemistry of South African Traditional Tonic Plants. Ph.D. Thesis, University of Johannesburg, Johannesburg, South Africa, 2012; p. 481.

41. Richter, T. Gut beraten mit Teufelskralle? Z. Phytother. 2001, 22, 43.

42. Schmidt, S. Die antiarthritische Wirkung der Harpagophytum-Wurzel. Österr. Apoth. Ztg. 1971, $25,829$.

43. Schmidt, S. Rheumatherapie mit Harpagophytum. Therapiewoche 1972, 22, 1072-1074.

44. Schmidt, S. Teufelskralle und Rheuma. Österr. Apoth. Ztg. 1983, 37, 111-113.

45. Scholz, H. Die Wurzel aus dem roten Sand. Kosmos 1977, 73, 122-124.

46. Schwabe, W. Übersicht über neuere Arzneipflanzen, die sich in den letzten 20 Jahren in der Homöopathie und der Phytotherapie bewährt haben. Allg. Homöopath. Ztg. 1980, 225, 217-229. [CrossRef]

47. Seeger, P.G. Harpagophytum, ein wirksames Phytotherapeutikum. Erfahrungsheilkunde 1973, 8, $255-256$.

48. Seeger, P.G. Harpagophytum-Ein wirksames Phytotherapeutikum. Naturheilpraxis 1973, 10, 488-492.

49. Sprecher, E. Problems with modern drugs: Ginseng-taiga root-Devil's claw. Schr. Bundesapothekerkamm. Wiss. Fortbild. Gelbe Reihe 1977, 5, 71-95.

50. Sticher, O. Die aktuelle Droge: Harpagophytum procumbens. Dtsch. Apoth. Ztg. 1977, 117, 1279-1284.

51. Vanhaelen, M. La biochimie et l'activite de Harpagophytum procumbens et de Glycyrrhiza glabra. Toxicite de Symphytum consolida. J. Pharm. Belg. 1986, 41, 172-182.

52. Vanhaelen, M.; Vanhaelen-Fastré, R.; Samaey-Fontaine, J.; Elchamid, A.; Niebes, P.; Matagne, D. Aspects botaniques, constitution chimique et activite pharmacologique d'Harpagophytum procumbens. Phytotherapy 1983, 5, 7-13.

53. Vogel, A.; Vogel, R. Die Teufelskralle (Harpagophytum). A. Vogel Gesundh. Nachr. 1988, 45, 54-55.

54. Vogel, A.; Vogel, S. Teufelskralle, Harpago. A. Vogel Gesundh. Nachr. 1973, 30, 102-104.

55. Vogel, A.; Vogel, S. Harpago, Teufelskralle. A. Vogel Gesundh. Nachr. 1978, 35, 42-43.

56. Vogel, A.; Vogel, S. Die Teufelskralle. A. Vogel Gesundh. Nachr. 1978, 35, 153-154.

57. Vogel, G. Wissenschaftliche Erkenntnisse zu Wirksamkeit und Unbedenklichkeit pflanzlicher Arzneimittel. Therapiewoche 1984, 34, 4078-4086.

58. Volk, O.H. Zur Kenntnis von Harpagophytum procumbens DC. Dtsch. Apoth. Ztg. 1964, 104, 573-576.

59. Voloshyn, O.I.; Smiyan, S.I.; Voloshyna, L.O.; Horevych, S.S. Использование мартинии душистой(Harpagophytum procumbens) в ревматологии: взгляд сквозь призму коморбидности(Обзор литературы). Semejnâ̂ Med. 2020, 3, 88-97. [CrossRef]

60. Wegener, T. Die Teufelskralle (Harpagophytum procumbens DC.) in der Therapie rheumatischer Erkrankungen. Z. Phytother. 1998, 19, 284-294.

61. Wegener, T. Wissenschaftliches Erkenntnismaterial zu Harpagophyti radix (Südafrikanische Teufelskralle) ab 1990—Unter Berücksichtigung Relevanter Früherer Studien; Kooperation Phytopharmaka: Bonn, Germany, 1998; p. 25.

62. Wegener, T. Devil's claw: From African traditional remedy to modern analgesic and antiinflammatory. HerbalGram 2000, 50, 47-54.

63. Wegener, T.; Winterhoff, H. Zubereitungen aus der südafrikanischen Teufelskralle. Dtsch. Apoth. Ztg. 2001, 141, $5613-5621$.

64. Wiss, H.-J. Was Wissen Wir über die Teufelskralle? Library of the Namibia Scientific Society: Windhoek, Namibia, $1974 ;$ p. 13.

65. Graner, G.; Lautenbacher, L. Harpagophytum procumbens DC (Teufelskralle); Kooperation Phytopharmaka: Bonn, Germany; p. 36.

66. Brossier, Y. Harpagophytum procumbens DC: Apport Bénéfique de la Phytothérapie dans le Traitement de la Maladie Inflammatoire Chronique. Ph.D. Thesis, Université Paul Sabatier, Toulouse, France, 1986; p. 163.

67. Mattern, B. L'Harpagophytum procumbens DC: Une Recente Acquisition de la Phytotherapie. Ph.D. Thesis, Universite de Bordeaux II, Bordeaux, France, 1983; p. 76.

68. Mundy, P.J.; Ncube, S.F. Devil's claw-A natural substitute for diclofenac? Vulture News 2014, 67, 43-47. [CrossRef]

69. Anonymous. Teufelskralle als pflanzliche Alternative. Ärztez. Nat. 1999, 40, 500.

70. Bonnefoy-Cudraz, Q. Le Droguier de la Faculté de Pharmacie de Montpellier (Sauvegarde du Patrimoine et Intérêt d'une des Plantes, 1 Harpagophytum procumbens Species). Ph.D. Thesis, Université de Montpellier I, Montpellier, France, 2013 ; p. 145.

71. Camponovo, F. Mise au Point de Procédés pour l'Analyse Phytochimique et Étude Comparative de Quelques Médicaments à Base de Ginkgo Biloba, Panax Ginseng et Harpagophytum procumbens. Ph.D. Thesis, Université de Lausanne, Lausanne, Switzerland, 1996; p. 289.

72. Couplan, F.; Danton, P. L'Harpagophytum. Un cadeau d'Afrique à menager. Rev. Monde Végétal 2000, 50, 16-19.

73. Ferrara, L.; Borrelli, F.; Borbone, N. Harpagophytum procumbens: New scientific evidences. In Proceedings of the 3rd International Symposium on Natural Drugs, Napoli, Italy, 2-4 October 2003. 
74. Fontanel, D. L’Harpagophytum. Lett. Phytothér. Rev. Inf. Pharm. Méd. 2005, 5, 1-6.

75. Franchi, G.G. Harpagophytum procumbens DC.: Una pianta africana entrata a far parte della medicina europea. Alcune osservazioni a carattere botanico, ecologico e farmacognostico. Piante Med. 2006, 5, 5-10.

76. Hadolt, H. Harpagophytum procumbens: Teufelskralle, Trampelklette. PhD of Thesis, Universität Wien, Wien, Austria, $1987 ;$ p. 57.

77. Kämpf, R. Harpagophytum procumbens DC, devil's claw. Schweiz. Apoth. 1976, 114, 337-342.

78. Quer, J.-C. Harpagophytum procumbens, Aspects Récents. PhD of Thesis, Université Descartes, Paris, France, $2007 ;$ p. 84.

79. Schmidt, T. Harpagophytum procumbens DC. Inf. Biol. Prax. 1972, 8, 21-23.

80. Smithies, S.J. Harpagophytum procumbens (Burch.) DC. Ex Meisn. Subsp. procumbens and Subsp. Transvaalense Ihlenf. \& HEK Hartmann (Pedaliaceae). 2006. Available online: http://opus.sanbi.org/bitstream/20.500.12143/3477/1/ Harpagophytumprocumbens_PlantzAfrica.pdf (accessed on 15 April 2021).

81. Burchell, W.J. Travels in the Interior of Southern Africa; Printed for Longman, Hurst, Rees, Orme, and Brown: London, UK, 1822; Volume 1, pp. 529-537.

82. Meisner, C.D.F. Plantarum Vascularium Genera: Secundum Ordines Naturales Digesta Eorumque Differentiae et Affinitates Tabulis Diagnostacis Expositae; Libraria Weidmannia: Leipzig, Germany, 1836-1843; Volume 1,2.

83. De Candolle, A. Prodromus Systematis Naturalis Regni Vegetabilis, Sive, Enumeratio Contracta Ordinum Generum Specierumque Plantarum Huc Usque Cognitarium, Juxta Methodi Naturalis, Normas Digesta: Pedalineae; Fortin, Masson et Sociorum: Paris, France, 1845; Volume 9, pp. 253-257.

84. Decaisne, M.J. Revue du groupe des pédalinées: Harpagophytum DC. Ann. Sci. Nat. Bot. 1865, 5, 321-336.

85. Mncwangi, N.; Vermaak, I.; Viljoen, A. Mid-infrared spectroscopy and short wave infrared hyperspectral imaging-A novel approach in the qualitative assessment of Harpagophytum procumbens and H. zeyheri (Devil's claw). Phytochem. Lett. 2014, 7, 143-149. [CrossRef]

86. Muzila, M. Genetic, Morphological and Chemical Variation in the Genus Harpagophytum. Ph.D. Thesis, Swedish University of Agricultural Sciences, Alnarp, Sweden, 2016; p. 76.

87. Muzila, M.; Setshogo, M.P.; Mpoloka, S.W. Multivariate analysis of Harpagophytum DC. Ex Meisn (Pedaliaceae) based on fruit characters. Int. J. Biodivers. Conserv. 2011, 3, 101-109.

88. Mncwangi, N.; Viljoen, A.; Vermaak, I.; Chen, W.; Zhang, J.; Khan, I.A. Metabolomic profiling and quality control of Harpagophytum spp. (Devil's claw). Planta Med. 2014, 80, CL1. [CrossRef]

89. Mosoabisane, M.F.T. Variation in chemical composition of Harpagophytum species as function of age and locality. Master's Thesis, University of the Free State, Bloemfontein, South Africa, 2009; p. 181.

90. Steenkamp, P.A.; Steenkamp, L.H. UPLC-MS profiling, identification of major peaks and comparison of Harpagophytum procumbens extracts from different locations. S. Afr. J. Bot. 2019, 124, 138-143. [CrossRef]

91. Hargreaves, B.J. The sesame family in Botswana. Botsw. Notes Rec. 1993, 25, 141-159.

92. Van Wyk, B.E.; Gericke, N. People's Plants-A Guide to Useful Plants of Southern Africa, 2nd, revised, and expanded ed.; Briza Publications: Queenswood, South Africa, 2018; pp. 174, 224.

93. Von Koenen, E. Heil-, Gift- und Essbare Pflanzen in Namibia; Klaus Hess Verlag: Göttingen, Germany, 1996; p. 336. ISBN 3980451828.

94. Smith, C.A. Common Names of South African Plants; Government Printer: Pretoria, South Africa, $1966 ;$ p. 642.

95. Ihlenfeldt, H.-D. Bemerkungen zur Taxonomie der süDwestafrikanischen Pedaliaceae; Mitteilungen der Botanischen Staatssammlung München Band VI.: München, Germany, 1967; pp. 593-612.

96. Baum, H. Kunene-Sambesi-Expedition; Otto Warburg, Verlag des Kolonial-Wirtschaftlichen Komitees: Berlin, Germany, 1903 ; p. 604.

97. Blank, R.J. Voraussetzungen und Möglichkeiten für Einen Feldmäßigen Anbau der Wildpflanze Harpagophytum procumbens (Teufelskralle). Master Thesis, Universität Hohenhein, Stuttgart-Hohenheim, Germany, 1973; p. 63.

98. Blank, R.J. Arbeiten und Berichte 19. Versuche zur Vermehrung von Harpagophytum procumbens DC. (Teufelskralle); Universität Hohenheim, Abteilung Pflanzenbau in den Tropen und Subtropen: Stuttgart-Hohenheim, Germany, 1976.

99. Von Willert, D.J.; Schneider, E. Teufelskralle: Anbau und Wildsammlung-Ein Beitrag zur pharmakognostischen Ökologie. Dtsch. Apoth. Ztg. 2001, 141, 683-688.

100. Wood, J.G. The Uncivilized Races, or Natural History of Man; American Publishing Company: Hartford, CT, USA, 1870; Volume 1, p. 783.

101. Cooke, M.C. Freaks and Marvels of Plant Life: Or Curiosities of Vegetation; Society for Promoting Christian Knowledge: London, UK, 1882; p. 463.

102. Lübbert, A. Aus dem deutsch-südwestafrikanischen Schutzgebiete. Ueber die Heilmethoden und Heilmittel der Eingeborenen in Deutsch-Südwestafrika. Mitth. Forsch. Gelehrt. Dtsch. Schutzgeb. 1901, 14, 77-90.

103. Hellwig, M. Angaben von Eingeborenen über die Feldkost und die Arzneipflanzen der Herrero und Hottentotten; Reichskolonialamt (Bundesarchiv R 1001/5989, fol. 78-81): Berlin, Germany, 1907.

104. Dinter, K. Die Vegetabilische Veldkost Deutsch-Südwest-Afrikas; Selbstverlag: Okahandja, Namibia, $1912 ;$ p. 24.

105. Dinter, K. Deutsch-Südwest-Afrika. Flora, Forst- und Landwirtschaftliche Fragmente; Weigel: Leipzig, Germany, 1909 ; p. 212.

106. Schön, A. Vom Pfeilgift zur Arznei: Untersuchungen von Arzneidrogen und Giften aus den Ehemaligen Deutschen Kolonien West- und Südwestafrikas, Vornehmlich an Berliner Instituten (1884-1918): Ein Beitrag zur Kolonialpharmazie; Wissenschaftliche Verlagsgesellschaft mbH: Stuttgart, Germany, 2017; p. 593. ISBN 3804737684.

107. Kroemer, B. Mit Schwert \& Pflugschar in Sachsen und Südwestafrika. Anekdoten und Geschichten Eines Südwester Pioniers: Gottreich Hubertus Mehnert; “Glanz \& Gloria” Verlag: Windhoek, Namibia, 2007; p. 128. ISBN 9789991668970. 
108. Anderson, S.; Staugard, F. Traditional Midwives (Traditional Medicine in Botswana); Ipelegeng Publishers: Gaborone, Botswana, 1986; p. 264, ISBN 978-9178103973.

109. Bieg, S. Beiträge Zur Kenntnis Einiger Heilpflanzen aus Deutsch-Südwestafrika mit Einer Liste der Dort Vorkommenden Medizinisch Verwendeten Pflanzen. Ph.D. Thesis, Technische Hochschule, Stuttgart, Germany, 1939; p. 49.

110. Van Damme, P.; van den Eynden, V.; Vernemmen, P. Plant uses by the Topnaar of the Sesfontein area (Namib desert). Afr. Focus 1992, 8. [CrossRef]

111. Watt, J.M.; Breyer-Brandwijk, M.G. The Medicinal and Poisonous Plants of Southern and Eastern Africa, 2nd ed.; Livingstone: London, UK, 1962; p. 1457.

112. Staugard, F. Traditional midwives in Botswana. Botsw. Natl. Health Bull. 1985, 1, 42-60. [PubMed]

113. Moreki, J.C. Documentation of ethnoveterinary practices used in family poultry in Botswana. Vet. World 2013, 6, 18-21. [CrossRef]

114. Maas, H. Außenminister Maas zum Abschluss der Verhandlungen mit Namibia. 2021. Available online: https://www. auswaertiges-amt.de/de/newsroom/- / 2463396 (accessed on 10 June 2021).

115. Kock, R. Erinnerungen an die Internierungszeit (1939-1946) und Zeitgeschichtliche Ergänzungen; Selbstverlag "Andalusia": Windhoek, Namibia, 1975; p. 209.

116. Zorn, B. Über die antiarthritische Wirkung der Harpagophytum-Wurzel. Dtsch. Rheumaforsch. 1958, 17, $134-138$.

117. Lux, R.E. Über ein Glukosid der Wurzel von Harpagophytum procumbens. Ph.D. Thesis, Julius-Maximilians-Universität, Würzburg, Germany, 1960; p. 75.

118. Stierstorfer, N. Ein Beitrag zur Kenntnis der Inhaltsstoffe von Harpagophytum procumbens DC. Ph.D. of Thesis, Julius-MaximiliansUniversität, Würzburg, Germany, 1961; p. 54.

119. Tunmann, P.; Lux, R.E. Zur chemischen Konstitution des Harpagosids. Pharm. Ztg. 1961, 106, 1357.

120. Tunmann, P.; Lux, R.E. Zur Kenntnis der Inhaltsstoffe aus der Wurzel von Harpagophytum procumbens DC. 1. Mitteilung: Isolierung und Eigenschaften der Glukoside Harpagosid und Harpagid. Dtsch. Apoth. Ztg. 1962, 102, 1274-1275.

121. Tunmann, P.; Lux, R.E. Zur chemischen Konstitution des Harpagosids. Dtsch. Apoth. Ztg. 1962, 101, 1383.

122. Fickentscher, K. Beitrag zur Chemischen Konstitution des Harpagids. Ph.D. Thesis, Julius-Maximilians-Universität, Würzburg, Germany, 1963; p. 63.

123. Tunmann, P.; Stierstorfer, N. Zur Kenntnis der Inhaltsstoffe aus der Wurzel von Harpagophytum procumbens DC. 2. Mitteilung. Dtsch. Apoth. Ztg. 1963, 103, 395-397.

124. Lichti, H.; von Wartburg, A. Zur Konstitution von Harpagosid. Tetrahedron Lett. 1964, 5, 835-843. [CrossRef]

125. Tunmann, P.; Stierstorfer, N. Zur Kenntnis der Inhaltsstoffe aus der Wurzel von Harpagophytum procumbens DC. Tetrahedron Lett. 1964, 5, 1697-1699. [CrossRef]

126. Lichti, H.; von Wartburg, A. Die Struktur des Harpagosids. 2. Mitteilung über Iridoide. Helv. Chim. Acta 1966, 49, 1552-1580. [CrossRef]

127. Hammer, H.-E. Konstitution des Procumbids und ein Beitrag zur Kenntnis Weiterer Inhaltsstoffe aus der Wurzel von Harpagophytum procumbens DC. Ph.D. Thesis, Julius-Maximilians-Universität, Würzburg, Germany, 1967; p. 65.

128. Tunmann, P.; Hammer, H.-E. Inhaltsstoffe aus der Wurzel von Harpagophytum procumbens DC., IV. Konstitution des Procumbids. Justus Liebigs Ann. Chem. 1968, 712, 138-145. [CrossRef]

129. Hagen, E. Correspondence Concerning the Trademarking of "Harpago"; Library of the Namibia Scientific Society: Windhoek, Namibia, 1961-1976; p. 10.

130. Schmidt, S. Phytotherapie beim rheumatischen Formenkreis. Arch. Arzneither. 1978, 3, $266-271$.

131. Beck, H.; Sand, J.-M.; Kuhlmann, J. Arzneimittelmarkt aktuell. Dtsch. Apoth. Ztg. 1981, 121, $2884-2889$.

132. Marandet, E. Harpagophytum procumbens DC. De l'Utilisation Traditionnelle à la Réglementation Européenne. Ph.D. Thesis, Université de Reims Champagne-Ardenne, Reims, France, 2009; p. 97.

133. Hagen, E. Harpagophytum. Letter to H.-J. Wiss Concerning Sustainability of Devil's Claw Exports to Germany; Library of the Namibia Scientific Society: Windhoek, Namibia, 1975; p. 2.

134. Achtnich, W. Harpagophytum. Letter to the South African Embassy in Germany (Cologne) Concerning Sustainability of Devil's Claw Exports to Germany; Library of the Namibia Scientific Society: Windhoek, Namibia, 1975; p. 2.

135. De Bruine, J.R.; Clark, D.L. A Short Revue of the Harpagophytum procumbens Problem; Department of Nature Conservation: Windhoek, Namibia, 1976.

136. Kgathi, D.L. The Grapple Plant Project. Seventh Progress Report: Aspects of Grapple Trade; University of Botswana: Gaborone, Botswana, 1987.

137. Kgathi, D.L. The grapple trade in Botswana. Botsw. Notes Rec. 1988, 20, 119-124.

138. Taylor, F.W.; Moss, H. Final Report on the Potential for Commercial Utilization of Veld Products. The Resource and its Management; Ministry of Commerce \& Industry: Gaborone, Botswana, 1982; Volume 1, p. 205.

139. Engels, G.; Brinckmann, J.A. Devil's claw—Harpagophytum procumbens, H. zeyheri. HerbalGram 2018, 118, 1-14.

140. Cunningham, A.B. African Medicinal Plants: Setting Priorities at the Interface Between Conservation and Primary Health Care; UNESCO: Paris, France, 1993.

141. Hachfeld, B. Analysis of the Trade Potential and Possible Over-Exploitation of a Southern African Medicinal Plant: Harpagophytum Procumbens; Bundesamt für Naturschutz: Bonn, Germany, 1999.

142. CITES. Biological And Trade Status of Harpagophytum. 2002, pp. 1-15. Available online: https://cites.org/sites/default/files/ eng/cop/12/doc/E12-46.pdf (accessed on 12 April 2021). 
143. Grote, K. The Increased Harvest and Trade of Devil's Claw (Harpagophytum procumbens) and Its Impacts on the Peoples and Environment of Namibia, Botswana and South Africa; Global Facilitation Unit for Underutilized Species: Maccarese, Italy, $2003 ;$ p. 30.

144. Hachfeld, B. Ecology and Utilisation of the Medicinal Plant Harpagophytum procumbens (Burch.) DC. ex Meissn. (Pedaliaceae) in Southern Africa; Bundesamt für Naturschutz: Bonn, Germany, 2004.

145. Hachfeld, B. Ecology and Utilisation of the Medicinal Plant Harpagophytum procumbens (Burch.) DC. ex Meissn. (Pedaliaceae) in Southern Africa. Ph.D. Thesis, Universität Hamburg, Hamburg, Germany, 2004; p. 305.

146. Cole, D.; Bennett, B. Trade, Poverty and Natural Products: Lessons Learned from Namibian Organic Devil'S Claw. 2007. Available online: http:/ / searchworks.stanford.edu/view/7838432 (accessed on 12 April 2021).

147. Hachfeld, B.; Schippmann, U. Conservation data sheet 2: Exploitation, trade and population status of Harpagophytum procumbens in southern Africa. Med. Plant Conserv. 2000, 6, 4-9.

148. Kathe, W.; Barsch, F.; Honnef, S. Trade in Devil's Claw (Harpagophytum spp.) in Germany—Status, Trends and Certification. 2003, pp. 1-40. Available online: http://foris.fao.org/static/pdf/NWFP/Germany_devils_claw.pdf (accessed on 13 April 2021).

149. Schippmann, U. Imports of Harpagophytum in Germany. In Proceedings of the First Regional Devil's Claw Conference, Windhoek, Namibia, 26-28 February 2002; p. 3.

150. Suckert, B. Successful marketing strategies as a tool for development. In Proceedings of the First Regional Devil's Claw Conference, Windhoek, Namibia, 26-28 February 2002; p. 2.

151. Censkowsky, U.; Helberg, U.; Nowack, A.; Steidle, M. Overview of World Production and Marketing of Organic Wild Collected Products; ITC: Geneva, Switzerland, 2007; p. 91.

152. Nott, K.; Nott, A.; Newton, D. A Critical Assessment of the Economic and Environmental Sustainability of the Namibian Indigenous Forest/Timber Industry with Reference to Zambia and Angola; TRAFFIC: Pretoria, South Africa, 2020; p. 101.

153. United States Pharmacopeial Convention. Harpagophytum Species Root. Proposed For Development Version 0.1. Herbal Medicines Compendium; USP: Rockville, MD, USA, 2013.

154. British Herbal Medicine Association. Harpagophytum. In British Herbal Pharmacopoeia, Part Three; BHMA: Cowling, UK, $1981 ;$ p. 49.

155. ANSM. Harpagophyton. Harpagophytum procumbens. In Pharmacopée Française, 10th ed.; Maisonneuve: Sainte-Ruffine, France, 1989; pp. 183-184.

156. Koch, H.P.; Hadold, H. Harpagophytum procumbens. Teufelskralle, Trampelklette; Kooperation Phytopharmaka: Bonn, Germany, $1988 ;$ p. 75.

157. Kommission, E. Harpagophyti radix (Berichtigung). Bundesanzeiger 1990, 164, 1.

158. ANSM. Extrait d'Harpagophyton (Sec). Harpagophyti extractum siccum. In Pharmacopée Française, 10th ed.; Maisonneuve: Sainte-Ruffine, France, 1992; pp. 1-4.

159. Bundesministerium für Gesundheit und Soziale Sicherung. Teufelskrallenwurzel. Harpagophyti radix. In Deutsches Arzneibuch (DAB) 10. 2. Nachtrag; Deutscher Apotheker Verlag: Stuttgart, Germany, 1993.

160. EDQM. Devil's claw root, Harpagophyti radix, 1997:1095. In European Pharmacopoeia, 3rd ed.; published June 1996, replaces the 2nd ed on 1 January 1997; Council of Europe: Strasbourg, France, 1997; p. 1821.

161. EDQM. Devil's claw root, Harpagophyti radix, 01/2003:1095. In European Pharmacopoeia, Supplement 4.3 to the Fourth Edition, published 20 June 2002; Council of Europe: Strasbourg, France, 2003; p. 359.

162. ANSM. Devil's claw root for homoeopathic preparations. Harpagophytum for homoeopathic preparations. Harpagophytum ad praeparationes homoeopathicas. In Pharmacopée Française, 10th ed.; Maisonneuve: Sainte-Ruffine, France, 2007; pp. 1-3.

163. EDQM. Devil's claw dry extract, Harpagophyti extractum siccum, 01/2008:1871. In European Pharmacopoeia, 7th ed.; Council of Europe: Strasbourg, France, 2008.

164. Health Canada. Devil's Claw-Harpagophytum. Nat. Health Prod. Ingred. Database 2021. Available online: http://webprod.hc-sc. gc.ca/nhpid-bdipsn/ingredReq.do?id=6188\&lang=eng (accessed on 12 April 2021).

165. EDQM. Devil's claw root, Harpagophyti radix, 01/2011:1095. In European Pharmacopoeia, 7th ed.; Council of Europe: Strasbourg, France, 2011.

166. Bacler-Żbikowska, B.; Drobnik, J. Komentarz botaniczny do roślin leczniczych i surowców roślinnych wymienionych w Farmakopei polskiej VIII. Część, I. Ann. Acad Med. Silesiensis 2011, 1-2, 48-60.

167. EMA. Assessment Report on Harpagophytum procumbens DC. and/or Harpagophytum zeyheri Decne., Radix. EMA/HMPC/627058/2015; Committee on Herbal Medicinal Products (HMPC): London, UK, 2016.

168. EMA. List of References Supporting the Assessment of Harpagophytum procumbens DC. and/or Harpagophytum zeyheri Decne., radix. EMA/HMPC/627059/2015; Committee on Herbal Medicinal Products (HMPC): London, UK, 2016.

169. EDQM. Devil's claw root, Harpagophyti radix, 01/2011:1095 corrected 9.6. In European Pharmacopoeia, 9th ed.; 6th supplement, Council of Europe: Strasbourg, France, 2018.

170. Kriukova, A.; Vladymyrova, I.; Gubar, S.; Kotov, A.; Kotova, E. Question introduction to the State Pharmacopoeia of Ukraine monograph «Devil's claw root». Manag. Econ. Qual. Assur. Pharm. 2018, 6-12. [CrossRef]

171. European Scientific Cooperative on Phytotherapy. Harpagophyti radix. In ESCOP Monographs. Fascicule 2; ESCOP: Exeter, UK, 1996; pp. 1-7.

172. Bradley, P.R. Addendum to the ESCOP monograph on Harpagophytum procumbens: Reply from ESCOP. Phytomedicine $2004,11,696$.

173. Chrubasik, S. Addendum to the ESCOP monograph on Harpagophytum procumbens. Phytomedicine 2004, 11, 696. [CrossRef]

174. World Health Organization. Radix Harpagophyti. In WHO Monographs on Selected Medicinal Plants; World Health Organization: Geneva, Switzerland, 2007; Volume 3, pp. 182-193, ISBN 9241545372. 
175. Anonymous. Harpagophytum procumbens. In African Herbal Pharmacopoeia; Brendler, T., Eloff, J.N., Gurib-Fakim, A., Phillips, L.D., Eds.; Association for African Medicinal Plants Standards Port Louis: Port Louis, Mauritius, 2010; pp. 127-134, ISBN 9990389098.

176. Devil's claw root. In Martindale: The Complete Drug Reference; Alison, B. (Ed.) The Pharmaceutical Press: London, UK, 2017; ISBN 978-0857113092.

177. Kemper, K.J.; Devil's Claw (Harpagophytum procumbens). Longwood Herb. Task Force Cent. Holist. Pediatr. Educ. Res. 1999, pp. 1-11. Available online: http:/ /ineldea.com.ua/images/devilsclaw.pdf (accessed on 13 April 2021).

178. Devil's claw. In Herbal Medicines; Barnes, J.; Baxter, I.A.; Smith, M.; Veitch, N.C. (Eds.) Pharmaceutical Press: London, UK, 2013; pp. 238-245, ISBN 9780857110350.

179. Edwards, S.E.; da Costa Rocha, I.; Williamson, E.M.; Heinrich, M. Devil's Claw Harpagophytum procumbens (Burch.) DC. Ex Meissner, H. zeyheri Decne. In Phytopharmacy: An Evidence-Based Guide to Herbal Medicinal Products; Willey Blackwell: Hoboken, NJ, USA, 2015; p. 131, ISBN 9781118543436.

180. Kooperation Phytopharmaka. Harpagophytum procumbens DC (Teufelskralle). Arzneipflanzenlexikon. 2020. Available online: https:/ / arzneipflanzenlexikon.info/teufelskralle.php (accessed on 12 April 2021).

181. Karioti, A.; Fani, E.; Vincieri, F.F.; Bilia, A.R. Analysis and stability of the constituents of Curcuma longa and Harpagophytum procumbens tinctures by HPLC-DAD and HPLC-ESI-MS. J. Pharm. Biomed. Anal. 2011, 55, 479-486. [CrossRef]

182. Mncwangi, N.; Viljoen, A.; Zhao, J.; Vermaak, I.; Chen, W.; Khan, I.A. What the devil is in your phytomedicine? Exploring species substitution in Harpagophytum through chemometric modeling of 1H-NMR and UHPLC-MS datasets. Phytochemistry 2014, 106, 104-115. [CrossRef]

183. Mncwangi, N.; Viljoen, A.M.; Vermaak, I.; Chen, W. Variation of the biologically active constituent harpagoside in Harpagophytum procumbens and H. zeyheri. Planta Med. 2013, 79, P58. [CrossRef]

184. Muzila, M.; Ekholm, A.; Nybom, H.; Widén, C.; Rumpunen, K. Harpagophytum germplasm varies in tuber peel and pulp content of important phenylpropanoids and iridoids. S. Afr. J. Bot. 2018, 115, 153-160. [CrossRef]

185. Koch, J.-K.-H. Über ein Chinon und Weitere Inhaltsstoffe aus der Wurzel von Harpagophytum procumbens DC. Ph.D. Thesis, Julius-Maximilians-Universität, Würzburg, Germany, 1970; p. 60.

186. Bianco, A.; Esposito, P.; Guiso, M.; Scarpati, M.L. Iridoids. 10. Procumbide, a diastereoisomer of antirrinoside. Gazz. Chim. Ital. 1971, 101, 764 .

187. Bauersfeld, H.-J. Über Weitere Inhaltsstoffe aus der Wurzel von Harpagophytum procumbens DC. Ph.D. Thesis, Julius-MaximiliansUniversität, Würzburg, Germany, 1974; p. 64.

188. Tunmann, P.; Bauersfeld, H.J. Über weitere Inhaltsstoffe der Wurzel von Harpagophytum procumbens DC. Arch. Pharm. 1975, 308, 655-657. [CrossRef] [PubMed]

189. Sticher, O. Plant mono-, di- and sesquiterpenoids with pharmacological or therapeutical activity. In New Natural Products and Plant Drugs with Pharmacological, Biological or Therapeutical Activity. In Proceedings of the First International Congress on Medicinal Plant Research, Section A, Munich, Germany, 6-10 September 1976; Wagner, H., Wolff, P., Eds.; Springer: Berlin/Heidelberg, Germany, 1977; pp. 137-176, ISBN 978-3-642-66684-1.

190. Kwasniewski, V. Beitrag zur Kenntnis der Inhaltsstoffe der Sekundärwurzeln von Harpagophytum procumbens DC. und zur Frage ihres eventuellen Ersatzes durch einheimische Drogen. Dtsch. Apoth. Ztg. 1978, 118, 49-50.

191. Bendall, M.R.; Ford, C.W.; Thomas, D.M. The structure of procumbide. Aust. J. Chem. 1979, 32, 2085-2091. [CrossRef]

192. Ziller, K.H.; Franz, G. Analysis of the water soluble fraction from the roots of Harpagophytum procumbens. Planta Med. 1979, 37, 340-348. [CrossRef]

193. Vanhaelen, M.; Vanhaelen Fastré, R.; Elchami, A.; Fontaine, J. Activité biologique d'Harpagophytum procumbens DC. I. Préparation et structure de l'harpagogénine. J. Pharm. Belg. 1981, 36, 38-42.

194. Franz, G.; Czygan, F.C.; Abou-Mandour, A.A. Untersuchungen der Gattung Harpagophytum. 4. Mitteilung: Gehalt an freien Zuckern und Harpagosid in Kalluskulturen und genuinen Wurzelgeweben von Harpagophytum procumbens. Planta Med. 1982, 44, 218-220. [CrossRef] [PubMed]

195. Kikuchi, T.; Matsuda, S.; Kubo, Y.; Namba, T. New iridoid glucosides from Harpagophytum procumbens DC. Chem. Pharm. Bull. 1983, 31, 2296-2301. [CrossRef]

196. Burger, J.F.W.; Brandt, E.V.; Ferreira, D. Iridoid and phenolic glycosides from Harpagophytum procumbens. Phytochemistry 1987, 26, 1453-1457. [CrossRef]

197. Baghdikian, B.; Ollivier, E.; Faure, R.; Debrauwer, L.; Rathelot, P.; Balansard, G. Two new pyridine monoterpene alkaloids by chemical conversion of a commercial extract of Harpagophytum procumbens. J. Nat. Prod. 1999, 62, 211-213. [CrossRef]

198. Picavet, S. Iridoides d'Harpagophytum procumbens DC (Plante a Propriete Anti-Rhumatismale). Ph.D. Thesis, Université de Reims Champagne-Ardenne, Reims, France, 2000; p. 31.

199. Boje, K. Phytochemische und Biopharmazeutische Untersuchungen an Harpagophytum procumbens DC. Ph.D. Thesis, Westfälische Wilhelms-Universität Münster, Münster, Germany, 2002; p. 286.

200. Clarkson, C.; Campbell, W.E.; Smith, P. In Vitro antiplasmodial activity of abietane and totarane diterpenes isolated from Harpagophytum procumbens (Devil's claw). Planta Med. 2003, 69, 720-724. [CrossRef] [PubMed]

201. Boje, K.; Lechtenberg, M.; Nahrstedt, A. New and known iridoid-and phenylethanoid glycosides from Harpagophytum procumbens and their in vitro inhibition of human leukocyte elastase. Planta Med. 2003, 69, 820-825. [CrossRef] 
202. Munkombwe, N.M. Acetylated phenolic glycosides from Harpagophytum procumbens. Phytochemistry 2003, 62, 1231-1234. [CrossRef]

203. Goroll, K.A. Pharmakologische Charakterisierung von Harpagosid, Einem Wirkstoff der Teufelskralle. Ph.D. Thesis, Universität Ulm, Ulm, Germany, 2004; p. 97.

204. Clarkson, C.; Staerk, D.; Hansen, S.H.; Smith, P.J.; Jaroszewski, J.W. Discovering new natural products directly from crude extracts by HPLC-SPE-NMR: Chinane diterpenes in Harpagophytum procumbens. J. Nat. Prod. 2006, 69, 527-530. [CrossRef]

205. Clarkson, C.; Staerk, D.; Hansen, S.H.; Smith, P.J.; Jaroszewski, J.W. Identification of major and minor constituents of Harpagophytum procumbens (Devil's claw) using HPLC-SPE-NMR and HPLC-ESIMS/APCIMS. J. Nat. Prod. 2006, 69, 1280-1288. [CrossRef]

206. Qi, J.; Chen, J.J.; Cheng, Z.H.; Zhou, J.H.; Yu, B.Y.; Qiu, S.X. Iridoid glycosides from Harpagophytum procumbens DC (Devil's claw). Phytochemistry 2006, 67, 1372-1377. [CrossRef]

207. Qi, J.; Zhou, J.; Chen, L.; Chen, J.J.; Yu, B.; Qiu, S. Study on chemical constituents in tuber of Harpagophytum procumbens DC. Chin. Pharm. J. 2006, 41, 1613-1615.

208. Qi, J.; Chen, J.; Tu, Y.; Chen, L.; Yu, B. Chemical constituents of African plant Harpagophytum procumbens. Chin. J. Nat. Med. 2007, 5, 105-107.

209. Wilken, D.; Röhnert, P.; Appel, K. Erschließung und Charakterisierung Therapeutisch Neuroprotektiver and Anti-Inflammatorischer Wirkstoffe aus Harpagophytum procumbens (Teufelskralle)—TheraTek. Abschlußbericht; BioPlanta GmbH: Leipzig, Germany, 2008 ; p. 41.

210. Appel, K.; Rose, T.; Fiebich, B.; Röhnert, P.; Claus, D.; Gerth, A.; Wilken, D. Neuroprotective and antiinflammatory effects of extracts from in vitro cultivated Harpagophytum procumbens (Devil's claw). Z. Phytother. 2009, 30, P03. [CrossRef]

211. Qi, J.; Li, N.; Zhou, J.H.; Yu, B.Y.; Qiu, S.X. Isolation and anti-inflammatory activity evaluation of triterpenoids and a monoterpenoid glycoside from Harpagophytum procumbens. Planta Med. 2010, 76, 1892-1896. [CrossRef] [PubMed]

212. Zgrajka, W.; Turska, M.; Rajtar, G.; Majdan, M.; Parada-Turska, J. Kynurenic acid content in anti-rheumatic herbs. Ann. Agric. Environ. Med. 2013, 20, 800-802. [PubMed]

213. Tomassini, L.; Serafini, M.; Foddai, S.; Ventrone, A.; Nicoletti, M. A new iridoid diglucoside from Harpagophytum procumbens. Nat. Prod. Res. 2016, 30, 157-161. [CrossRef] [PubMed]

214. Schmidt, S. “Teufelskralle” vielfach bakteriell bedenklich. Dtsch. Apoth. Ztg. 1978, 118, 1808-1809.

215. Abramowicz, M. Toxic reactions to plant products sold in health food stores. Med. Lett. Drugs Ther. 1979, 21, $29-31$.

216. Loew, D.; Schuster, O.; Möllerfeld, J. Stabilität und biopharmazeutische Qualität. Voraussetzung für Bioverfügbarkeit und Wirksamkeit von Harpagophytum procumbens. In Phytopharmaka II, Forschung und Klinische Anwendung; Loew, D., Rietbrock, N., Eds.; Steinkopff: Darmstadt, Germany, 1996; pp. 83-93.

217. Chrubasik, S. Biopharmazeutische Qualität und klinische Wirksamkeit von Zubereitungen aus Harpagophytum Extrakt. In Rheumatherapie mit Phytopharmaka; Chrubasik, S., Wink, M., Eds.; Hippokrates: Stuttgart, Germany, 1997; pp. $77-85$.

218. Schier, W.; Bauersfeld, H.-J. Handelssorten von Harpagophytum procumbens DC. 1. Mitteilung. Dtsch. Apoth. Ztg. 1973, 113, 795-796.

219. Schier, W. Handelssorten von Harpagophytum procumbens DC. 2. Mitteilung. Dtsch. Apoth. Ztg. 1974, 114, 1800-1801.

220. Becker, H.; Richter, S. Eine einfache dünnschichtchromatographische Untersuchung von Harpagophytum procumbens für das Apotheken-Labor. Pharm. Ztg. 1975, 120, 441-442.

221. Czygan, F.-C.; Krüger, A. Pharmazeutisch-biologische Untersuchungen der Gattung Harpagophytum-3. Mitteilung: Zur Verteilung des Iridoid-Glycosids Harpagosid in den einzelnen Organen von Harpagophytum procumbens DC und Harpagophytum zeyheri Decne. Planta Med. 1977, 31, 305-307. [CrossRef]

222. Haag-Berrurier, M.; Kuballa, B.; Anton, R. Dosage des glucoiridoïdes totaux dans la racine d'Harpagophytum procumbens DC. Plantes Méd. Phytothér. 1978, 12, 197-206.

223. Sticher, O.; Meier, B. Quantitative Bestimmung von Harpagosid in Wurzeln von Harpagophytum procumbens mit Hochleistungsflüssigkeitschromatographie (HPLC). Dtsch. Apoth. Ztg. 1980, 120, 1592-1594.

224. Vanhaelen, M.; Vanhaelen-Fastré, R.; Elchami, A.A. Gas-liquid chromatographic determination of the iridoid content in Harpagophytum procumbens DC. J. Chromatogr. A 1981, 209, 476-478. [CrossRef]

225. Ragusa, S.; Circosta, C.; Galati, E.M.; Tumino, G. A drug used in traditional medicine. Harpagophytum procumbens DC I. Scanning electron microscope observations. J. Ethnopharmacol. 1984, 11, 245-257. [CrossRef] [PubMed]

226. Pourrat, H.; Texier, O.; Vennat, B.; Pourrat, A.; Galliard, J. Etude de la stabilité des iridoïdes d'Harpagophytum procumbens DC au cours de la préparation de poudres et d'atomisats. Ann. Pharm. Fr. 1985, 43, 601-606. [PubMed]

227. Ficarra, P.; Ficarra, R.; Tommasini, A.; De Pasquale Costa, R.; Guarniera Fenech, C.; Ragusa, S. Analisi mediante HPLC di una droga della medicina tradizionale: Harpagophytum procumbens DC. Nota I. Boll. Chim. Farm. 1986, 125, 250-253. [PubMed]

228. Franke, A.; Rimpler, H. GC/MS-Analyse methylierter Iridoidglykoside. Planta Med. 1986, 52, 89-95. [CrossRef]

229. Guillerault, L.; Ollivier, E.; Elias, R.; Balansard, G. Determination of harpagide, 8-para-coumaroyl harpagide, and harpagoside by high performance liquid chromatography in Harpagophytum procumbens drugs and in a commercial extract. J. Liq. Chromatogr. 1994, 17, 2951-2960. [CrossRef]

230. Mestdagh, O.; Torck, M. Etude de la qualité de gélules d'Harpagophyton. Ann. Pharm. Fr. 1995, 53, 135-137. [PubMed]

231. Wolf, J. Mikro-Dünnschichtchromatographie Teufelskrallenwurzel. Pharm. Ztg. 1995, 140, 28.

232. Chrubasik, S.; Sporer, F.; Wink, M. Zum Harpagosidgehalt verschiedener Trockenextraktpulver aus Harpagophytum procumbens. Complement. Med. Res. 1996, 3, 6-11. [CrossRef] 
233. Chrubasik, S.; Sporer, F.; Wink, M. Zum Wirkstoffgehalt in Teezubereitungen aus Harpagophytum procumbens. Complement. Med. Res. 1996, 3, 116-119. [CrossRef]

234. Chrubasik, S.; Sporer, F.; Wink, M. Zum Wirkstoffgehalt in Arzneimitteln aus Harpagophytum procumbens. Complement. Med. Res. 1996, 3, 57-63. [CrossRef]

235. Poukens-Renwart, P.; Tits, M.; Angenot, L. Quantitative densitometric evaluation of harpagoside in the secondary roots of Harpagophytum procumbens DC. J. Planar Chromatogr. 1996, 9, 199-202.

236. Marotta, M.; Addabbo, I.; Kosasi, S. The quality control and stability testing of homeopathic preparations. Boll. Chim. Farm. 1998, $137,439-441$.

237. Sporer, F.; Chrubasik, S. Präparate aus der Teufelskralle (Harpagophytum procumbens). Z. Phytother. 1999, 20, 335-336.

238. Chrubasik, S.; Sporer, F.; Dillmann-Marschner, R.; Friedmann, A.; Wink, M. Physicochemical properties of harpagoside and its in vitro release from Harpagophytum procumbens extract tablets. Phytomedicine 2000, 6, 469-473. [CrossRef]

239. Schneider, E.; Sanders, J.; Von Willert, D.J. Vermeidung von Verfälschungen der Teufelskralle Harpagophytum procumbens: Ein Beitrag zur pharmakognostischen Ökologie. Drogenreport 2001, 14, 12-16.

240. Chrubasik, S.; Pollak, S.; Fiebich, B. Harpagophytum extracts. Clin. Pharm. 2002, 71, 104-105. [CrossRef] [PubMed]

241. Gray, B.R. Retention of the Harpagoside Content in Dried Harpagophytum procumbens (Devil's Claw) Root Through Controlled Drying and the Application of Near Infrared Spectroscopy (NIRS) as Rapid Method of Determination. Master's Thesis, University of Stellenbosch, Stellenbosch, South Africa, 2003; p. 138.

242. Baranska, M.; Schulz, H.; Siuda, R.; Strehle, M.A.; Rosch, P.; Popp, J.; Joubert, E.; Manley, M. Quality control of Harpagophytum procumbens and its related phytopharmaceutical products by means of NIR-FT-Raman spectroscopy. Biopolymers 2005, 77, 1-8. [CrossRef]

243. Günther, M.; Schmidt, P.C. Comparison between HPLC and HPTLC-densitometry for the determination of harpagoside from Harpagophytum procumbens CO(2)-extracts. J. Pharm. Biomed. Anal. 2005, 37, 817-821. [CrossRef]

244. Joubert, E.; Manley, M.; Gray, B.R.; Schulz, H. Rapid measurement and evaluation of the effect of drying conditions on harpagoside content in Harpagophytum procumbens (devil's claw) root. J. Agric. Food Chem. 2005, 53, 3493-3502. [CrossRef]

245. Schmidt, A.H. Fast HPLC for quality control of Harpagophytum procumbens by using a monolithic silica column: Method transfer from conventional particle-based silica column. J. Chromatogr. A 2005, 1073, 377-381. [CrossRef] [PubMed]

246. Schmidt, A.H. Validation of a fast-HPLC method for the separation of iridoid glycosides to distinguish between the Harpagophytum species. J. Liq. Chromatogr. Relat. Technol. 2007, 28, 2339-2347. [CrossRef]

247. Seger, C.; Godejohann, M.; Tseng, L.H.; Spraul, M.; Girtler, A.; Sturm, S.; Stuppner, H. LC-DAD-MS/SPE-NMR hyphenation. A tool for the analysis of pharmaceutically used plant extracts: Identification of isobaric iridoid glycoside regioisomers from Harpagophytum procumbens. Anal. Chem. 2005, 77, 878-885. [CrossRef]

248. Zucchi, O.L.; Moreira, S.; de Jesus, E.F.; Neto, H.S.; Salvador, M.J. Characterization of hypoglycemiant plants by total reflection X-ray fluorescence spectrometry. Biol. Trace Elem. Res. 2005, 103, 277-290. [CrossRef]

249. Arranz, I.; Sizoo, E.; van Egmond, H.; Kroeger, K.; Legarda, T.M.; Burdaspal, P.; Reif, K.; Stroka, J. Determination of aflatoxin B1 in medical herbs: Interlaboratory study. J. AOAC Int. 2006, 89, 595-605. [CrossRef] [PubMed]

250. Colas, C.; Garcia, P.; Popot, M.A.; Bonnaire, Y.; Bouchonnet, S. Liquid chromatography/electrospray ionization mass spectrometric characterization of Harpagophytum in equine urine and plasma. Rapid Commun. Mass Spectrom. 2006, 20, $3257-3266$. [CrossRef] [PubMed]

251. Colas, C.; Bouchonnet, S.; Rogalewicz-Gilard, F.; Popot, M.A.; Ohanessian, G. Proton and sodium cation affinities of harpagide: A computational study. J. Phys. Chem. A 2006, 110, 7503-7508. [CrossRef]

252. Schmidt, A.H.; Schiemann, U. Teufelskralle aus dem Drogeriemarkt oder aus der Apotheke? Dtsch. Apoth. Ztg. 2006, 146, 50-55.

253. Spriano, D.; Krasniqi, B.; Strompen, T.; Tobler, M.; Meier, B. The drug-extract-ratio of aqueous/ethanolic Harpagophyti radix extracts has to be revised. Planta Med. 2006, 72, P_287. [CrossRef]

254. Street, R.A.; Southway, C.; Stirk, W.A.; Van Staden, J. Determination of mineral elements and heavy metals in indigenous medicinal plants of KwaZulu-Natal. S. Afr. J. Bot. 2007, 73, 315. [CrossRef]

255. Colas, C.; Popot, M.-A.; Garcia, P.; Bonnaire, Y.; Bouchonnet, S. Analysis of iridoids from Harpagophytum and eleutherosides from Eleutherococcus senticosus in horse urine. Biomed. Chromatogr. 2008, 22, 912-917. [CrossRef]

256. Colas, C.; Garcia, P.; Popot, M.-A.; Bonnaire, Y.; Bouchonnet, S. Optimization of solid-phase extraction for the liquid chromatography-Mass spectrometry analysis of harpagoside, 8-para-coumaroyl harpagide, and harpagide in equine plasma and urine. J. Chromatogr. Sci. 2008, 46, 174-183. [CrossRef]

257. Wagner, S.; Urena, A.; Reich, E.; Merfort, I. Validated HPTLC methods for the determination of salicin in Salix sp. and of harpagoside in Harpagophytum procumbens. J. Pharm. Biomed. Anal. 2008, 48, 587-591. [CrossRef] [PubMed]

258. Chigome, S.; Nindi, M.; Munkombwe, N. Quality control of Harpagophytum procumbens products using High Pressure Liquid Chromatography-Diode Array Detection (HPLC-DAD). Niger. J. Nat. Prod. Med. 2009, 13, 26-29. [CrossRef]

259. Babili, F.E.; Fouraste, I.; Rougaignon, C.; Moulis, C.; Chatelain, C. Anatomical study of secondary tuberized roots of Harpagophytum procumbens DC and quantification of harpagoside by high-performance liquid chromatography method. Pharm. Mag. 2012, 8, 175-180. [CrossRef]

260. Newmaster, S.G.; Grguric, M.; Shanmughanandhan, D.; Ramalingam, S.; Ragupathy, S. DNA barcoding detects contamination and substitution in North American herbal products. BMC Med. 2013, 11, 222. [CrossRef] [PubMed] 
261. Naidoo, Y.; Heneidak, S.; Bhatt, A.; Kasim, N.; Naidoo, G. Morphology, histochemistry, and ultrastructure of foliar mucilageproducing trichomes of Harpagophytum procumbens (Pedaliaceae). Turk. J. Bot. 2014, 38, 60-67. [CrossRef]

262. Zhao, J.; Mncwangi, N.; Viljoen, A.; Wang, M.; Khan, I.A. Differentiation of Harpagophytum procumbens and H. zeyheri through NMR-based chemometric approach. Planta Med. 2014, 80, PPL17. [CrossRef]

263. Kondamudi, N.; Turner, M.W.; McDougal, O.M. Harpagoside content in devil's claw extracts. Nat. Prod. Commun. 2016, 11, 1215-1216. [CrossRef] [PubMed]

264. Kriukova, A.; Vladymyrova, I. The definition of numeric indicators for the root of Harpagophytum procumbens. In Proceedings of the Topical Issues of New Drugs Development: XXIII International Scientific and Practical Conference of Young Scientists and Students, Kharkiv, Ukraine, 21 April 2016; p. 188.

265. Baghdikian, B.; Filly, A.; Fabiano-Tixier, A.-S.; Petitcolas, E.; Mabrouki, F.; Chemat, F.; Ollivier, É. Extraction by solvent using microwave and ultrasound-assisted techniques followed by HPLC analysis of Harpagoside from Harpagophytum procumbens and comparison with conventional solvent extraction methods. C. R. Chim. 2016, 19, 692-698. [CrossRef]

266. Diuzheva, A.; Carradori, S.; Andruch, V.; Locatelli, M.; De Luca, E.; Tiecco, M.; Germani, R.; Menghini, L.; Nocentini, A.; Gratteri, P.; et al. Use of innovative (micro)extraction techniques to characterise Harpagophytum procumbens root and its commercial food supplements. Phytochem. Anal. 2018, 29, 233-241. [CrossRef] [PubMed]

267. Kriukova, A.; Vladymyrova, I.; Tishakova, T. Rationale for choosing of extraction solvent for obtaining liquid extract from the roots of Harpagophytum procumbens DC. Scr. Sci. Pharm. 2017, 4, 37.

268. Kriukova, A.; Vladymyrova, I. The GC-MS determination of chemical constituents from Harpagophytum procumbens DC roots. Technol. Transf. Innov. Solut. Med. 2017, 52-54. [CrossRef]

269. Pretorius, E.; Van der Bank, M.; Viljoen, A.M. DNA Barcoding detects contamination and substitution in herbal products containing Harpagophytum spp. S. Afr. J. Bot. 2017, 109, 364-365. [CrossRef]

270. Avato, P.; Argentieri, M.P. Quality assessment of commercial spagyric tinctures of Harpagophytum procumbens and their antioxidant properties. Molecules 2019, 24, 2251. [CrossRef] [PubMed]

271. Nalluri, B.N.; Sujithkumar, S. Characterization and estimation of harpagoside in dried root extract and oral powder formulations of Harpagophytum procumbens by validated RP-HPLC-PDA method. J. Drug Deliv. Ther. 2019, 9, 38-46. [CrossRef]

272. De Aragao Tannus, C.; de Souza Dias, F.; Santana, F.B.; Dos Santos, D.; Magalhaes, H.I.F.; de Souza Dias, F.; de Freitas Santos Junior, A. Multielement determination in medicinal plants and herbal medicines containing Cynara scolymus L., Harpagophytum procumbens DC, and Maytenus ilifolia (Mart.) ex Reiss from Brazil using ICP OES. Biol. Trace Elem. Res. 2020. [CrossRef] [PubMed]

273. Dos Santos Ribeiro, G.; de Assis Carneiro, A.; Martins, D.H.N.; Simeoni, L.A.; Silveira, D.; Magalhães, P.O.; Fonseca-Bazzo, Y.M Determination of harpagoside in Harpagophytum procumbens DC tablet's using analytical method by high performance liquid chromatography. Eclét. Quím. J. 2020, 45, 47-55. [CrossRef]

274. Duband, F. Harpagophytum procumbens DC: Recherche d'une Activité Anti-Inflammatoire Aiguë. Ph.D. Thesis, UFR de Pharmacie, Clermont-Ferrand, France, 1986; p. 76.

275. Plaizier-Vercammen, J.A.; Bruwier, C. Evaluation of excipients for direct compression of the spray-dried extract of Harpagophytum procumbens. Soc. Fr. Sci. Tech. Pharm. 1986, 2, 525-530.

276. Günther, M.; Maus, M.; Wagner, K.G.; Schmidt, P.C. Hydrophilic solutes in modified carbon dioxide extraction-prediction of the extractability using molecular dynamic simulation. Eur. J. Pharm. Sci. 2005, 25, 321-329. [CrossRef]

277. Piechota-Urbanska, M.; Kolodziejska, J.; Berner-Strzelczyk, A. Zastosowanie wyciagu z czarciego pazura w przeciwzapalnych preparatach aplikowanych na skórę, wytworzonych na bazie polimerów kwasu akrylowego. Polim. Med. 2009, 39, 9-15.

278. Almajdoub, S.S. Polymer Coating of an Optimized Nano Lipid Carrier System of Harpagophytum procumbens Extract for Oral Delivery. Master's Thesis, University of the Western Cape, Cape Town, South Africa, 2017; p. 182.

279. Lopes, A.P.; Bagatela, B.S.; Lopes, I.P.; Gregorio, L.E.; Maistro, E.L.; Fonseca, F.L.A.; Perazzo, F.F. Production of gastro-resistant coated tablets prepared from the hydroethanolic standardized roots extract of Harpagophytum procumbens DC. Afr. J. Pharm. Pharmacol. 2017, 11, 491-500. [CrossRef]

280. Villasenor, I.M. Bioactivities of iridoids. Anti. Inflamm. Anti. Allergy Agents Med. Chem. (Former. Curr. Med. Chem. Anti Inflamm. Anti Allergy Agents) 2007, 6, 307-314. [CrossRef]

281. Serrano, A.; Ros, G.; Nieto, G. Bioactive compounds and extracts from traditional herbs and their potential anti-inflammatory health effects. Medicines 2018, 5, 76. [CrossRef] [PubMed]

282. Fontaine, J.; Elchami, A.; Vanhaelen, M.; Vanhaelen Fastré, R. Activité biologique d'Harpagophytum procumbens DC. 2. Analyse pharmacologique des effets de l'harpagoside, l'harpagide et l'harpagogenine sur l'ileon isole de cobaye. J. Pharm. Belg. 1981, 36, 321-324.

283. Benito, P.B.; Lanza, A.M.D.; Sen, A.M.S.; De Santos Galindez, J.; Matellano, L.F.; Gómez, A.S.; Martínez, M.J.A. Effects of some iridoids from plant origin on arachidonic acid metabolism in cellular systems. Planta Med. 2000, 66, 324-328. [CrossRef] [PubMed]

284. Fiebich, B.L.; Heinrich, M.; Hiller, K.O.; Kammerer, N. Inhibition of TNF-alpha synthesis in LPS-stimulated primary human monocytes by Harpagophytum extract SteiHap 69. Phytomedicine 2001, 8, 28-30. [CrossRef]

285. Loew, D.; Mollerfeld, J.; Schrodter, A.; Puttkammer, S.; Kaszkin, M. Investigations on the pharmacokinetic properties of Harpagophytum extracts and their effects on eicosanoid biosynthesis in vitro and ex vivo. Clin. Pharm. 2001, 69, 356-364. [CrossRef]

286. Wahrendorf, M.S.; Sporer, F.; Wink, M. Anti-inflammation assays on Harpagophytum procumbens. Des Sources du Savoir aux Médicaments du Futur: Actes du 4e Congrès Européen d’Ethnopharmacologie. In Proceedings of the 4th European Congress on 
Ethnopharmocology, Metz, France, 11-13 May 2000; Fleurentin, J., Pelt, J., Mazars, G., Lejosne, J.C., Cabalion, P., Eds.; IRD: Metz, France, 2002; pp. 399-401, ISBN 2709915049.

287. Jang, M.H.; Lim, S.; Han, S.M.; Park, H.J.; Shin, I.; Kim, J.W.; Kim, N.J.; Lee, J.S.; Kim, K.A.; Kim, C.J. Harpagophytum procumbens suppresses lipopolysaccharide-stimulated expressions of cyclooxygenase-2 and inducible nitric oxide synthase in fibroblast cell line L929. J. Pharm. Sci. 2003, 93, 367-371. [CrossRef] [PubMed]

288. Schulze-Tanzil, G.; Hansen, C.; Shakibaei, M. Wirkung des Extraktes aus Harpagophytum procumbens DC auf MatrixMetalloproteinasen in menschlichen Knorpelzellen in vitro. Arzneimittelforschung 2004, 54, 213-220. [CrossRef] [PubMed]

289. Chrubasik, J.E. Zur Knorpelprotektion von Harpagophytum procumbens DC: Histologische, Zellbiologische und Molekularbiologische Untersuchungen. Ph.D. Thesis, Justus-Liebig-Universität Gießen, Gießen, Germany, 2006; p. 135.

290. Chrubasik, J.E.; Neumann, E.; Lindhorst, E.; Chrubasik, S.; Muller-Ladner, U. Evaluation of the chondroprotective effect of Harpagophytum procumbens. Med. Klin. 2006, 101, A90.

291. Hadzhiyski, H.; Torda, T.; Chrubasik, S.; Lindhorst, E.; Raif, W. Impact of Harpagophytum procumbens on the urinary pyridinoline deoxypyridinoline ratio in experimental osteoarthritis. Focus Altern. Complement. Ther. 2006, 11, 13. [CrossRef]

292. Günther, M.; Laufer, S.; Schmidt, P.C. High anti-inflammatory activity of harpagoside-enriched extracts obtained from solventmodified super- and subcritical carbon dioxide extractions of the roots of Harpagophytum procumbens. Phytochem. Anal. 2006, 17, 1-7. [CrossRef] [PubMed]

293. Huang, T.H.; Tran, V.H.; Duke, R.K.; Tan, S.; Chrubasik, S.; Roufogalis, B.D.; Duke, C.C. Harpagoside suppresses lipopolysaccharideinduced iNOS and COX-2 expression through inhibition of NF-kappa B activation. J. Ethnopharmacol. 2006, 104, 149-155. [CrossRef]

294. Balthazar, L.v.; Eggenschwiler, J.; Rohrer, J.; Suter, A. Investigations on the antiinflammatory way of action of a Harpagophytum extract using microarray technology. Planta Med. 2009, 75, PJ158. [CrossRef]

295. Ebrahim, N.; Uebel, R.A. Direct inhibition of cyclooxygenase-2 enzyme by an extract of Harpagophytum procumbens, harpagoside and harpagide. Afr. J. Pharm. Pharmacol. 2011, 5, 2209-2212. [CrossRef]

296. Gyurkovska, V.; Alipieva, K.; Maciuk, A.; Dimitrova, P.; Ivanovska, N.; Haas, C.; Bley, T.; Georgiev, M. Anti-inflammatory activity of Devil's claw in vitro systems and their active constituents. Food Chem. 2011, 125, 171-178. [CrossRef]

297. Fiebich, B.L.; Munoz, E.; Rose, T.; Weiss, G.; McGregor, G.P. Molecular targets of the antiinflammatory Harpagophytum procumbens (Devil's claw): Inhibition of TNFalpha and COX-2 gene expression by preventing activation of AP-1. Phytother. Res. 2012, 26, 806-811. [CrossRef] [PubMed]

298. Ebrahim, N. Development and Characterization of a Transdermal Formula for an Extract of the Medicinal Plant Harpagophytum procumbens. Ph.D. Thesis, University of the Western Cape, Belville, South Africa, 2013; p. 199.

299. Hostanska, K.; Melzer, J.; Rostock, M.; Suter, A.; Saller, R. Alteration of anti-inflammatory activity of Harpagophytum procumbens (Devil's claw) extract after external metabolic activation with S9 mix. J. Pharm. Pharmacol. 2014, 66, 1606-1614. [CrossRef]

300. Haseeb, A.; Leigh, D.; Haqqi, T.M. A small molecule harpagoside inhibits IL-1beta-induced expression of IL-6 by blocking the expression of C-FOS in primary human osteoarthritis chondrocytes. Osteoarthr. Cartil. 2015, 23, A155-A156. [CrossRef]

301. Haseeb, A.; Ansari, M.Y.; Haqqi, T.M. Harpagoside suppresses IL-6 expression in primary human osteoarthritis chondrocytes. J. Orthop. Res. 2017, 35, 311-320. [CrossRef]

302. Kim, T.K.; Park, K.S. Inhibitory effects of harpagoside on TNF-alpha-induced pro-inflammatory adipokine expression through PPAR-gamma activation in 3T3-L1 adipocytes. Cytokine 2015, 76, 368-374. [CrossRef] [PubMed]

303. Schopohl, P.; Gruneberg, P.; Melzig, M.F. The influence of harpagoside and harpagide on TNFalpha-secretion and cell adhesion molecule mRNA-expression in IFNgamma/LPS-stimulated THP-1 cells. Fitoterapia 2016, 110, 157-165. [CrossRef] [PubMed]

304. Mariano, A.; Di Sotto, A.; Leopizzi, M.; Garzoli, S.; Di Maio, V.; Gulli, M.; Dalla Vedova, P.; Ammendola, S.; Scotto d'Abusco, A. Antiarthritic effects of a root extract from Harpagophytum procumbens DC: Novel insights into the molecular mechanisms and possible bioactive phytochemicals. Nutrients 2020, 12, 2545. [CrossRef]

305. Eichler, O.; Koch, C. Über die antiphlogistische, analgetische und spasmolytische Wirksamkeit von Harpagosid, einem Glykosid aus der Wurzel von Harpagophytum procumbens DC. Drug Res. 1970, 20, 107-109.

306. Anonymous. Prüfung von "Harpagophytum procumbens DC" auf Antiphlogistische Wirksamkeit am Rattenpfotenödem; International Bio Research, Mitteilung an Fa. Hagen: Freilassing, Germany, 1974.

307. Anonymous. Subakute Studie an der Ratte zur Prüfung des Einflusses von Harpagosan-Extrakt auf Verschiedene Blutkomponente; International Bio Research, Mitteilung an Fa. Hagen: Freilassing, Germany, 1974.

308. Erdös, A.; Fontaine, R.; Friehe, H.; Durand, R.; Pöppinghaus, T. Beitrag zur Pharmakologie und Toxikologie verschiedener Extrakte, sowie des Harpagosids aus Harpagophytum procumbens DC. Planta Med. 1978, 34, 97-108. [CrossRef]

309. McLeod, D.W.; Revell, P.; Robinson, B.V. Investigations of Harpagophytum procumbens (Devil's claw) in the treatment of experimental inflammation and arthritis in the rat. Br. J. Pharmacol. 1979, 66, 140P-141P.

310. Manez, S.; Alcaraz, M.; Paya, M.; Rios, J.; Hancke, J. Selected extracts from medicinal plants as anti-inflammatory agents. Planta Med. 1990, 56, 656. [CrossRef]

311. Lanhers, M.C.; Fleurentin, J.; Mortier, F.; Vinche, A.; Younos, C. Anti-inflammatory and analgesic effects of an aqueous extract of Harpagophytum procumbens. Planta Med. 1992, 58, 117-123. [CrossRef] [PubMed]

312. Jadot, G.; Lecomte, A. Activité anti-inflammatoire d'Harpagophytum procumbens DC. Lyon Méditerr. Méd. Méd. Sud. Est. 1992, $28,833-835$. 
313. Del Carmen Recio, M.; Giner, R.M.; Máñez, S.; Ríos, J.L. Structural considerations on the iridoids as anti-inflammatory agents. Planta Med. 1994, 60, 232-234. [CrossRef] [PubMed]

314. Soulimani, R.; Younos, C.; Mortier, F.; Derrieu, C. The role of stomachal digestion on the pharmacological activity of plant extracts, using as an example extracts of Harpagophytum procumbens. Can. J. Physiol. Pharmacol. 1994, 72, 1532-1536. [CrossRef] [PubMed]

315. Abe, S.; Ishibashi, H.; Masuo, K.; Tanaka, S.-I.; Yamaguchi, H. Suppression of carrageenan-induced edema by oral administration of extracts of Uncaria tomentosa and/or Harpagophytum procumbens. Oyo Yakuri Pharmacomet. 2002, 62, 27-32.

316. Andersen, M.L.; Santos, E.H.; Seabra Mde, L.; da Silva, A.A.; Tufik, S. Evaluation of acute and chronic treatments with Harpagophytum procumbens on Freund's adjuvant-induced arthritis in rats. J. Ethnopharmacol. 2004, 91, 325-330. [CrossRef]

317. Mahomed, I.M.; Ojewole, J.A.O. Analgesic, antiinflammatory and antidiabetic properties of Harpagophytum procumbens DC (Pedaliaceae) secondary root aqueous extract. Phytother. Res. 2004, 18, 982-989. [CrossRef]

318. Mahomed, I.M. Some Pharmacological Properties of Harpagophytum procumbens DC [Pedaliaceae] Secondary Root Extract. Ph.D. Thesis, University of KwaZulu-Natal, Durban, South Africa, 2004; p. 172.

319. Ahmed, M.I.; Afifi, M.I.; Younos, I.H. Harpagophytum procumbens (Devil's claw): A possible natural anti-inflammatory agent (an experimental study). IJPT 2005, 4, 54-63.

320. Kundu, J.K.; Mossanda, K.S.; Na, H.K.; Surh, Y.J. Inhibitory effects of the extracts of Sutherlandia frutescens (L.) R. Br. and Harpagophytum procumbens DC. on phorbol ester-induced COX-2 expression in mouse skin: AP-1 and CREB as potential upstream targets. Cancer Lett. 2005, 218, 21-31. [CrossRef] [PubMed]

321. Catelan, S.C.; Belentani, R.M.; Marques, L.C.; Silva, E.R.; Silva, M.A.; Caparroz-Assef, S.M.; Cuman, R.K.; Bersani-Amado, C.A. The role of adrenal corticosteroids in the anti-inflammatory effect of the whole extract of Harpagophytum procumbens in rats. Phytomedicine 2006, 13, 446-451. [CrossRef]

322. Chrubasik, J.E.; Lindhorst, E.; Neumann, E.; Gerlach, U.; Faller-Marquardt, M.; Torda, T.; Muller-Ladner, U.; Chrubasik, S. Potential molecular basis of the chondroprotective effect of Harpagophytum procumbens. Phytomedicine 2006, 13, 598-600. [CrossRef] [PubMed]

323. Uchida, S.; Hirai, K.; Hatanaka, J.; Hanato, J.; Umegaki, K.; Yamada, S. Antinociceptive effects of St. John's wort, Harpagophytum procumbens extract and Grape seed proanthocyanidins extract in mice. Biol. Pharm. Bull. 2008, 31, 240-245. [CrossRef] [PubMed]

324. Wachsmuth, L.; Lindhorst, E.; Wrubel, S.; Hadzhiyski, H.; Hudelmaier, M.; Eckstein, F.; Chrubasik, S. Micro-morphometrical assessment of the effect of Harpagophytum procumbens extract on articular cartilage in rabbits with experimental osteoarthritis using magnetic resonance imaging. Phytother. Res. 2011, 25, 1133-1140. [CrossRef] [PubMed]

325. Wrubel, S. Untersuchungen zur Wirkung von Harpagophytum procumbens auf Kniegelenksarthrose im Kaninchen unter Verwendung der Magentresonanztomographie. Ph.D. Thesis, Ludwig-Maximilians-Universität, München, Germany, 2014 ; p. 65.

326. Bisinotto, R. Efeito Anti-Inflamatório do Extrato Etanólico da Harpagophytum procumbens Durante a Inflamação Intestinal De Camundongos Infectados Com Salmonella Enteritidis (ATCC13076). Master's Thesis, Universidade Federal de São Carlos, São Carlos, Brazil, 2014; p. 100.

327. Lim, D.W.; Kim, J.G.; Han, D.; Kim, Y.T. Analgesic effect of Harpagophytum procumbens on postoperative and neuropathic pain in rats. Molecules 2014, 19, 1060-1068. [CrossRef] [PubMed]

328. Parenti, C.; Arico, G.; Chiechio, S.; Di Benedetto, G.; Parenti, R.; Scoto, G.M. Involvement of the heme-oxygenase pathway in the antiallodynic and antihyperalgesic activity of Harpagophytum procumbens in Rats. Molecules 2015, 20, 16758-16769. [CrossRef]

329. Ucuncu, Y.; Celik, N.; Ozturk, C.; Turkoglu, M.; Cetin, N.; Kockara, N.; Sener, E.; Dundar, C.; Arslan, A.; Dogan, H.; et al. Chondroprotective effects of a new glucosamine combination in rats: Gene expression, biochemical and histopathological evaluation. Life Sci. 2015, 130, 31-37. [CrossRef]

330. Parenti, C.; Arico, G.; Pennisi, M.; Venditti, A.; Scoto, G.M. Harpagophytum procumbens extract potentiates morphine antinociception in neuropathic rats. Nat. Prod. Res. 2016, 30, 1248-1255. [CrossRef]

331. Radomska-Lesniewska, D.M.; Skopinska-Rozewska, E.; Demkow, U.; Jozwiak, J.; Sobiecka, M.; Balan, B.J. A natural herbal remedy modulates angiogenic activity of bronchoalveolar lavage cells from sarcoidosis patients. Cent. Eur. J. Immunol. 2016, 41, 25-34. [CrossRef]

332. Tippler, B.; Syrovets, T.; Plaza, N.; Loew, D.; Simmet, T.H. Harpagophytum procumbens DC used in traditional medicine inhibits eicosanoid biosynthesis in human whole blood. Int. J. Tissue React. 1997, 19, 101.

333. Tippler, B.; Syrovets, T.; Loew, D.; Simmet, T.H. Harpagophytum procumbens: Wirkung von Extrakten auf die Eicosanoidbiosynthese in lonophor A23187-stimuliertem menschlichem Vollblut. In Phytopharmaka II, Forschung und Klinische Anwendung; Loew, D., Rietbrock, N., Eds.; Steinkopff: Darmstadt, Germany, 1996; pp. 95-100.

334. Anauate, M.C. Efeito Dos Extratos De Harpagohytum procumbens (Garra-do-Diabo) E Suas Frações Na Atividade da COX-1 e COX-2 e Na Produção De NO Em Sangue Total. Ph.D. Thesis, Universidade de São Paulo, São Paulo, Brazil, 2007 ; p. 98.

335. Abdelouahab, N.; Heard, C.M. Effect of the major glycosides of Harpagophytum procumbens (Devil's claw) on epidermal cyclooxygenase-2 (COX-2) in vitro. J. Nat. Prod. 2008, 71, 746-749. [CrossRef] [PubMed]

336. Abdelouahab, N.; Heard, C.M. Dermal and transcutaneous delivery of the major glycoside constituents of Harpagophytum procumbens (Devil's claw) in vitro. Planta Med. 2008, 74, 527-531. [CrossRef] [PubMed]

337. Ouitas, N.A.; Heard, C.M. Topical Delivery and Effects of Harpagophytum procumbens. Ph.D. Thesis, Cardiff University, Cardiff, UK, 2009; p. 298. 
338. Ouitas, N.A.; Heard, C.M. A novel ex vivo skin model for the assessment of the potential transcutaneous anti-inflammatory effect of topically applied Harpagophytum procumbens extract. Int. J. Pharm. 2009, 376, 63-68. [CrossRef]

339. Ouitas, N.A.; Heard, C.M. Estimation of the relative antiinflammatory efficacies of six commercial preparations of Harpagophytum procumbens (Devil's claw). Phytother. Res. 2010, 24, 333-338. [CrossRef]

340. Anauate, M.C.; Torres, L.M.; de Mello, S.B. Effect of isolated fractions of Harpagophytum procumbens DC (Devil's claw) on COX-1, COX-2 activity and nitric oxide production on whole-blood assay. Phytother. Res. 2010, 24, 1365-1369. [CrossRef] [PubMed]

341. Whitehouse, L.W.; Znamirowska, M.; Paul, C.J. Devil's claw (Harpagophytum procumbens): No evidence for anti-inflammatory activity in the treatment of arthritic disease. Can. Med. Assoc. J. 1983, 129, 249-251.

342. Na, H.K.; Mossanda, K.S.; Lee, J.Y.; Surh, Y.J. Inhibition of phorbol ester-induced COX-2 expression by some edible African plants. Biofactors 2004, 21, 149-153. [CrossRef]

343. Inaba, K.; Murata, K.; Naruto, S.; Matsuda, H. Inhibitory effects of devil's claw (secondary root of Harpagophytum procumbens) extract and harpagoside on cytokine production in mouse macrophages. J. Nat. Med. 2010, 64, 219-222. [CrossRef] [PubMed]

344. Rahimi, A.; Razmkhah, K.; Mehrnia, M.; Mohamadnia, A.; Sahebjamee, H.; Salehi, S.; Asl, E.A.; Tahmasebi, H.; Shandiz, S.A.S.; Davouodbeglou, F.; et al. Molecular docking and binding study of harpagoside and harpagide as novel anti-inflammatory and anti-analgesic compound from Harpagophytum procumbens based on their interactions with COX-2 enzyme. Asian Pac. J. Trop. Dis. 2016, 6, 227-231. [CrossRef]

345. Locatelli, M.; Ferrante, C.; Carradori, S.; Secci, D.; Leporini, L.; Chiavaroli, A.; Leone, S.; Recinella, L.; Orlando, G.; Martinotti, S.; et al. Optimization of aqueous extraction and biological activity of Harpagophytum procumbens root on ex vivo rat colon inflammatory model. Phytother. Res. 2017, 31, 937-944. [CrossRef]

346. Leporini, L.; Ferrante, C.; Recinella, L.; Orlando, G.; Chiavaroli, A.; Martinotti, S.; Carradori, S.; Locatelli, M.; Vecchiotti, G.; Menghini, L. Evaluation of protective effect of Harpagophytum procumbens DC. Ex Meisn. Root water extraction by microwave, biological activity on ex vivo rat colon inflammatory model and microscopic investigation. In Proceedings of the $112^{\circ}$ Congresso della Società Botanica Italiana, IV International Plant Science Conference (IPSC), Parma, Italy, 20-23 September 2017 ; p. 1.

347. Cholet, J.; Decombat, C.; Vareille-Delarbre, M.; Gainche, M.; Berry, A.; Ogéron, C.; Ripoche, I.; Delort, L.; Vermerie, M.; Fraisse, D. Comparison of the anti-inflammatory and immunomodulatory mechanisms of two medicinal herbs: Meadowsweet (Filipendula ulmaria) and Harpagophytum (Harpagophytum procumbens). Int. J. Plant Anim. Environ. Sci. 2019, 9, 145-163.

348. Recinella, L.; Chiavaroli, A.; Ronci, M.; Menghini, L.; Brunetti, L.; Leone, S.; Tirillini, B.; Angelini, P.; Covino, S.; Venanzoni, R.; et al. Multidirectional pharma-toxicological study on Harpagophytum procumbens DC. Ex Meisn.: An IBD-focused investigation. Antioxidants 2020, 9, 168. [CrossRef] [PubMed]

349. Grant, L.; McBean, D.E.; Fyfe, L.; Warnock, A.M. The inhibition of free radical generation by preparations of Harpagophytum procumbens in vitro. Phytother. Res. 2009, 23, 104-110. [CrossRef]

350. Kaszkin, M.; Beck, K.F.; Koch, E.; Erdelmeier, C.; Kusch, S.; Pfeilschifter, J.; Loew, D. Downregulation of iNOS expression in rat mesangial cells by special extracts of Harpagophytum procumbens derives from harpagoside-dependent and independent effects. Phytomedicine 2004, 11, 585-595. [CrossRef]

351. Bae, Y.H.; Cuong, T.D.; Hung, T.M.; Kim, J.A.; Woo, M.H.; Byeon, J.S.; Choi, J.S.; Min, B.S. Cholinesterase inhibitors from the roots of Harpagophytum procumbens. Arch. Pharm. Res. 2013, 37, 1124-1129. [CrossRef]

352. Bhattacharya, A.; Bhattcharya, S.K. Anti-oxidant activity of Harpagophytum procumbens (Devil's claw). Br. J. Phytother. 1998, 5, 68-71.

353. Langmead, L.; Dawson, C.; Hawkins, C.; Banna, N.; Loo, S.; Rampton, D.S. Antioxidant effects of herbal therapies used by patients with inflammatory bowel disease: An in vitro study. Aliment Pharm. Ther. 2002, 16, 197-205. [CrossRef]

354. Betancor-Fernandez, A.; Perez-Galvez, A.; Sies, H.; Stahl, W. Screening pharmaceutical preparations containing extracts of turmeric rhizome, artichoke leaf, devil's claw root and garlic or salmon oil for antioxidant capacity. J. Pharm. Pharmacol. 2003, 55, 981-986. [CrossRef] [PubMed]

355. Almeida, M.C.; Soares, S.F.; Abreu, P.R.; Jesus, L.M.; Brito, L.C.; Bernardo-Filho, M. Protective effect of an aqueous extract of Harpagophytum procumbens upon Escherichia coli strains submitted to the lethal action of stannous chloride. Cell. Mol. Biol. 2007, 53, OL923-927.

356. Grant, L. The Putative Anti-Inflammatory and Analgesic Properties of Harpagophytum procumbens. Ph.D. Thesis, Queen Margaret University College, Edinburgh, UK, 2005; p. 350.

357. Georgiev, M.; Alipieva, K.; Pashova, S.; Denev, P.; Angelova, M.; Kerns, G.; Bley, T. Antioxidant activity of devil's claw cell biomass and its active constituents. Food Chem. 2010, 121, 967-972. [CrossRef]

358. Georgiev, M.; Alipieva, K.; Orhan, I.E. Cholinesterases inhibitory and antioxidant activities of Harpagophytum procumbens from in vitro systems. Phytother. Res. 2011, 26, 313-316. [CrossRef]

359. Schaffer, L.F.; Peroza, L.R.; Boligon, A.A.; Athayde, M.L.; Alves, S.H.; Fachinetto, R.; Wagner, C. Harpagophytum procumbens prevents oxidative stress and loss of cell viability in vitro. Neurochem. Res. 2013, 38, 2256-2267. [CrossRef]

360. Schaffer, L.F. Efeito Do Harpagophytum procumbens Sobre Parâmetros De Estresse Oxidativo E a Viabilidade Celular In Vitro. Master's Thesis, Universidade Federal De Santa Maria, Santa Maria, Brazil, 2013; p. 62.

361. Muzila, M.; Rumpunen, K.; Wright, H.; Roberts, H.; Grant, M.; Nybom, H.; Sehic, J.; Ekholm, A.; Widen, C. Alteration of neutrophil reactive oxygen species production by extracts of Devil's claw (Harpagophytum). Oxid. Med. Cell. Longev. 2016, 2016, 3841803. [CrossRef] [PubMed] 
362. Schaffer, L.F.; de Freitas, C.M.; Chiapinotto Ceretta, A.P.; Peroza, L.R.; de Moraes Reis, E.; Krum, B.N.; Busanello, A.; Boligon, A.A.; Sudati, J.H.; Fachinetto, R.; et al. Harpagophytum procumbens ethyl acetate fraction reduces fluphenazine-induced vacuous chewing movements and oxidative stress in rat brain. Neurochem. Res. 2016, 41, 1170-1184. [CrossRef]

363. Mosca, F.; Marruchella, G.; Mariani, F.; Tiscar, P.G. Effetto dell'arpagoside sul burst respiratorio dei neutrofili di suino. In Proceedings of the XLIII Meeting Annuale SIPAS, Reggio Emilia, Italy, 16-17 March 2017; pp. 191-195.

364. Ungerer, G.; Cui, J.; Ndam, T.; Bekemeier, M.; Song, H.; Li, R.; Siedhoff, H.R.; Yang, B.; Appenteng, M.K.; Greenlief, C.M.; et al. Harpagophytum procumbens extract ameliorates allodynia and modulates oxidative and antioxidant stress pathways in a rat model of spinal cord injury. Neuromol. Med. 2020, 22, 278-292. [CrossRef]

365. Ncube, S.F.; McGaw, L.J.; Njoya, E.M.; Ndagurwa, H.G.T.; Mundy, P.G.; Sibanda, S. In Vitro antioxidant activity of crude extracts of Harpagophytum zeyheri and their anti-inflammatory and cytotoxicity activity compared with diclofenac. BMC Complement. Altern. Med. 2021, in press. [CrossRef]

366. Mahomed, I.M.; Nsabimana, A.M.; Ojewole, J.A.O. Pharmacological effects of Harpagophytum procumbens DC [Pedaliaceae] secondary root aqueous extract on isolated gastro-intestinal tract muscles of the chick, guinea-pig and rabbit. Afr. J. Pharm. Pharmacol. 2005, 2, 31-45. [CrossRef]

367. Guérin, J.C.; Réveillère, H.P. Activité antifongique d'extraits végétaux à usage thérapeutique. II. Étude de 40 extraits sur 9 souches fongiques. Ann. Pharm. Fr. 1985, 43, 77-81. [PubMed]

368. Weckesser, S.; Engel, K.; Simon-Haarhaus, B.; Wittmer, A.; Pelz, K.; Schempp, C.M. Screening of plant extracts for antimicrobial activity against bacteria and yeasts with dermatological relevance. Phytomedicine 2007, 14, 508-516. [CrossRef] [PubMed]

369. Oliveira, S.R.P. Avaliação do efeito do extrato etanólico bruto de Harpagophytum procumbens em camundongos infectados com Toxocara canis. Ropositório Inst. UFSCar. 2012. Available online: https:/ / repositorio.ufscar.br/handle/ufscar/7011 (accessed on 13 April 2021).

370. Camillo, L.; Oliveira, S.R.P.; Ribeiro, R.D.; Rodolpho, J.M.A.; Caroccia, G.H.G.; Albuquerque, S.; Anibal, F.F. Efeito de bioterápico na eosinofilia durante a SLMV experimental. Rev. Ciênc. Farm. Básica Apl. 2014, 35, 701-708.

371. Oliveira, S.R.P.; Rodolpho, J.M.A.; Dejani, N.D.; Souza, L.C.; Correia, R.O.; Neris, D.M.; Galvão, A.; de Matos, A.P.K.; Vieira, P.C.; Afonso, A.; et al. Harpagophytum procumbens modulates eosinophilic response during infection by Toxocara canis. Int. J. Recent Sci. Res. 2014, 5, 2008-2013.

372. Correia, R. Ação Do Tratamento Com Mentha piperita L. e Harpagophytum procumbens Contra Schistosoma mansoni-In Vitro: Análise Proteômica. Ph.D. Thesis, Universidade Federal de São Carlos, São Carlos, Brazil, 2014; p. 122.

373. Cock, I.E.; Bromley, A. Inhibition of bacterial triggers of selected autoimmune inflammatory diseases by Harpagophytum procumbens Burch. DC. Ex Meisn. fruit extracts. S. Afr. J. Bot. 2017, 100, 329. [CrossRef]

374. Luigi, M. Etude des Propriétés Antimutagènes de l'Harpagophytum procumbens et de l'Harpagoside: Généralisation aux Plantes Anti-Inflammatoires. Ph.D. Thesis, Ecole Doctorale Sciences de l’Environnement, Aix-Marseille, France, $2014 ;$ p. 340.

375. Luigi, M.; Baghdikian, B.; Orsière, T.; Pompili, J.; Mabrouki, F.; Ollivier, E.; Botta, A. Antimutagenic potential of harpagoside and Harpagophytum procumbens against 1-nitropyrene. Pharmacogn. Mag. 2015, 11, S29-S36. [CrossRef]

376. Kim, J.Y.; Park, S.H.; Baek, J.M.; Erkhembaatar, M.; Kim, M.S.; Yoon, K.H.; Oh, J.; Lee, M.S. Harpagoside inhibits RANKL-induced osteoclastogenesis via Syk-Btk-PLCgamma2-Ca(2+) signaling pathway and prevents inflammation-mediated bone loss. J. Nat. Prod. 2015, 78, 2167-2174. [CrossRef] [PubMed]

377. Chung, H.J.; Kyung Kim, W.; Joo Park, H.; Cho, L.; Kim, M.R.; Kim, M.J.; Shin, J.S.; Ho Lee, J.; Ha, I.H.; Kook Lee, S. Antiosteoporotic activity of harpagide by regulation of bone formation in osteoblast cell culture and ovariectomy-induced bone loss mouse models. J. Ethnopharmacol. 2016, 179, 66-75. [CrossRef] [PubMed]

378. Chung, H.J.; Kim, W.K.; Oh, J.; Kim, M.R.; Shin, J.S.; Lee, J.; Ha, I.H.; Lee, S.K. Anti-osteoporotic activity of Harpagoside by upregulation of the BMP2 and Wnt signaling pathways in osteoblasts and suppression of differentiation in osteoclasts. J. Nat. Prod. 2017, 80, 434-442. [CrossRef]

379. Vollmann, D. Zu Einigen Pharmakologischen Wirkungen von Zubereitungen des Harpagophytum procumbens DC. Ph.D. Thesis, Karl-Marx-Universität, Leipzig, Germany, 1965; p. 34.

380. Circosta, C.; Occhiuto, F.; Ragusa, S.; Trovato, A.; Tumino, G.; Briguglio, F.; De Pasquale, A. A drug used in traditional medicine: Harpagophytum procumbens DC II. Cardiovascular activity. J. Ethnopharmacol. 1984, 11, 259-274. [CrossRef]

381. De Pasquale, R.; Circosta, C.; Iauk, L.; Ragusa, S.; Busa, G. Effetti dell' Harpagophytum procumbens DC e dell'arpagoside sulle aritmie sperimentali "in vitro". Pharm. Mediterr. 1984, 15, 153.

382. De Pasquale, R.; Busa, G.; Circosta, C.; Iauk, L.; Ragusa, S.; Ficarra, P.; Occhiuto, F. A drug used in traditional medicine: Harpagophytum procumbens DC. III. Effects on hyperkinetic ventricular arrhythmias by reperfusion. J. Ethnopharmacol. 1985, 13, 193-199. [CrossRef]

383. Occhiuto, F.; Circosta, C.; Ragusa, S.; Ficarra, P.; Costa De Pasquale, R. A drug used in traditional medicine: Harpagophytum procumbens DC. IV. effects on some isolated muscle preparations. J. Ethnopharmacol. 1985, 13, 201-208. [CrossRef]

384. Occhiuto, F.; De Pasquale, A. Electrophysiological and haemodynamic effects of some active principles of Harpagophytum procumbens DC. in the dog. Pharm. Res. 1990, 22, 72-73. [CrossRef]

385. Mahomed, I.M.; Ojewole, J.A.O. Cardiovascular effects of Harpagophytum procumbens DC. Afr. J. Tradit. Complement. Altern. Med. 2004, 1, 30-44. 
386. Mahomed, I.M.; Ojewole, J.A.O. Anticonvulsant activity of Harpagophytum procumbens DC [Pedaliaceae] secondary root aqueous extract in mice. Brain Res. Bull. 2006, 69, 57-62. [CrossRef] [PubMed]

387. Ferrante, C.; Recinella, L.; Locatelli, M.; Guglielmi, P.; Secci, D.; Leporini, L.; Chiavaroli, A.; Leone, S.; Martinotti, S.; Brunetti, L.; et al. Protective effects induced by microwave-assisted aqueous Harpagophytum extract on rat cortex synaptosomes challenged with amyloid beta-peptide. Phytother. Res. 2017, 31, 1257-1264. [CrossRef] [PubMed]

388. Chen, C.; Zhang, H.; Xu, H.; Xue, R.; Zheng, Y.; Wu, T.; Lian, Y. Harpagoside rescues the memory impairments in chronic cerebral hypoperfusion rats by inhibiting PTEN activity. J. Alzheimers Dis. 2018, 63, 445-455. [CrossRef] [PubMed]

389. Peruru, R.; Usha Rani, R.; Thatiparthi, J.; Sampathi, S.; Dodoala, S.; Prasad, K. Devil's claw (Harpagophytum procumbens) ameliorates the neurobehavioral changes and neurotoxicity in female rats exposed to arsenic. Heliyon 2020, 6, e03921. [CrossRef]

390. Prosinska, J.; Sawicka, T.; Drozd, J. Investigation of the thymomimetic activity of a selected phytopharmaceutical preparationReumaherb tablets by flow cytometry. Acta Pol. Pharm. 2002, 59, 265-274.

391. Torres-Fuentes, C.; Theeuwes, W.F.; McMullen, M.K.; McMullen, A.K.; Dinan, T.G.; Cryan, J.F.; Schellekens, H. Devil's claw to suppress appetite-Ghrelin receptor modulation potential of a Harpagophytum procumbens root extract. PLoS ONE 2014, 9, e103118. [CrossRef] [PubMed]

392. Saleh, S.; Jahromi, H.K.; Sarikhani, Y.; Jahromi, Z.K.; Dowlatkhah, H. The effects of hydroalcoholic extract of devil's claw on serum levels of obestatin and body weight in male rats. J. Glob. Pharma Technol. 2016, 12, 40-43.

393. Anonymous. Der Einfluß von Harpagosan (Harpagophytum procumbens DC) auf die Blei-Einlagerung in Rattenorganen; International Bio Research, Mitteilung an Fa. Hagen: Freilassing, Germany, 1975.

394. Chrubasik, S.; Wink, M. Zur pharmakologischen Wirkung der Teufelskralle (Harpagophytum procumbens). Complement. Med. Res. 1995, 2, 323-325. [CrossRef]

395. Loew, D.; Kaszkin, M. Experimentell- und klinisch-pharmakologische Modelle zum Nachweis der analgetischen und antiphlogistischen Wirkungen von Teufelskralle. Ärztez. Nat. 2002, 43, 576-585.

396. Fleurentin, J.; Mortier, F. Entzündungshemmende und analgetische Wirkungen von Harpagophytum procumbens und H. zeyheri. In Rheumatherapie mit Phytopharmaka; Chrubasik, S., Wink, M., Eds.; Hippokrates: Stuttgart, Germany, 1997; pp. 68-76.

397. Budzinski, J.W.; Foster, B.C.; Vandenhoek, S.; Arnason, J.T. An in vitro evaluation of human cytochrome P450 3A4 inhibition by selected commercial herbal extracts and tinctures. Phytomedicine 2000, 7, 273-282. [CrossRef]

398. Puttkammer, S. Untersuchungen zur Pharmakokinetik des Harpagophytum-Spezialextraktes HF 8858 und Einfluß auf Leukotrien C4-Biosynthese Sowie die Thromboxan B2-Freisetzung. Ph.D. Thesis, Johann Wolfgang Goethe-Universität Frankfurt/M., Frankfurt, Germany, 2000; p. 45.

399. Hilgendorf, C.; Döppenschmitt, S. Popular herbal drugs: Screening of potential CYP inhibition/activation based metabolic interactions. Int. J. Clin. Pharm. 2003, 41, 537.

400. Unger, M.; Frank, A. Simultaneous determination of the inhibitory potency of herbal extracts on the activity of six major cytochrome P450 enzymes using liquid chromatography/mass spectrometry and automated online extraction. Rapid Commun. Mass Spectrom. 2004, 18, 2273-2281. [CrossRef]

401. Romiti, N.; Tramonti, G.; Corti, A.; Chieli, E. Effects of Devil's claw (Harpagophytum procumbens) on the multidrug transporter ABCB1/P-glycoprotein. Phytomedicine 2009, 16, 1095-1100. [CrossRef] [PubMed]

402. Modarai, M.; Suter, A.; Kortenkamp, A.; Heinrich, M. The interaction potential of herbal medicinal products: A luminescencebased screening platform assessing effects on cytochrome P450 and its use with devil's claw (Harpagophyti radix) preparations. J. Pharm. Pharmacol. 2011, 63, 429-438. [CrossRef]

403. Anton, R. Réflexions sur quelques nouvelles acquisitions en phytothérapie. J. Pharm. Belg. 1987, 42, $138-151$.

404. Möse, J.R. Untersuchung der Toxischen Wirkung von Harpago-Tee auf Zellen und Dessen Verhalten in Einem Orientierenden Versuch am Tumor-Tier; Hygiene-Institut, Universität Graz. Mitteilung an Fa. Hagen: Freilassing, Germany, 1969.

405. Grünewald, K. Erfahrungen mit Harpagosan bei Hypercholesterinämie und Hyperurikämie. Arch. Arzneither. 1978, 1, 73-76.

406. Anonymous. Akute Toxizitätsprüfung von Harpagophytum D2 nach Intravenöser Applikation an der Ratte; International Bio Research, Mitteilung an Fa. Hagen: Freilassing, Germany, 1974.

407. Anonymous. Akute Toxizitätsprüfung von Harpagophytum procumbens DC nach Oraler Applikation bei der Ratte; International Bio Research, Mitteilung an Fa. Hagen: Freilassing, Germany, 1975.

408. Ibrahim, K.E.; Al-Ashban, R.M.; El-Sammani, S.A. Toxicity studies on devil's claw herbal medicine. Res. J. Pharmacol. 2010, 4, 69-73. [CrossRef]

409. Al-Harbi, N.O.; Al-Ashban, R.M.; Shah, A.H. Toxicity studies on Harpagophytum procumbens (Devil's claw) capsules in mice. J. Med. Plants Res. 2013, 7, 3089-3097. [CrossRef]

410. Allard, T.; Wenner, T.; Greten, H.J.; Efferth, T. Mechanisms of herb-induced nephrotoxicity. Curr. Med. Chem. 2013, 20, 2812-2819. [CrossRef] [PubMed]

411. Joshi, K.; Parrish, A.; Grunz-Borgmann, E.A.; Gerkovich, M.; Folk, W.R. Toxicology studies of aqueous-alcohol extracts of Harpagophytum procumbens subsp. procumbens (Burch.) DC. Ex Meisn. (Pedaliaceae) in female and male rats. BMC Complement. Altern. Med. 2020, 20, 9. [CrossRef]

412. Mahomed, I.M.; Ojewole, J.A.O. Oxytocin-like effect of Harpagophytum procumbens DC [Pedaliaceae] secondary root aqueous extract on rat isolated uterus. Afr. J. Tradit. Complement. Altern. Med. 2006, 3, 82-89. 
413. Mahomed, I.M.; Ojewole, J.A. Uterotonic effect of Harpagophytum procumbens DC (Pedaliaceae) secondary root aqueous extract on rat isolated uterine horns. J. Smooth Muscle Res. 2009, 45, 231-239. [CrossRef]

414. Pearson, W. Reproductive Toxicity Study of an Herbal Product (Nasprin) in Sprague Dawley Rats; Nutraceutical Alliance: Burlington, ON, Canada, 2012.

415. Davari, S.A. Teratogenic effects of Harpagophytum procumbens ethanolic extract in mice and fetuses. Zahedan J. Res. Med. Sci. 2016, 18, e3481. [CrossRef]

416. Chrubasik, S.; Ziegler, R. Wirkstoffgehalt in Arzneimitteln aus Harpagophytum procumbens und klinische Wirksamkeit von Harpagophytum-Trockenextrakt. In Phytopharmaka II, Forschung und Klinische Anwendung; Loew, D., Rietbrock, N., Eds.; Steinkopff: Darmstadt, Germany, 1996; pp. 101-114.

417. Brendler, T.; Grünwald, J.; Ulbricht, C.; Basch, E. Devil's claw (Harpagophytum procumbens DC): An evidence-based systematic review by the Natural Standard Research Collaboration. J. Herb. Pharm. 2006, 6, 89-126. [CrossRef]

418. Büechi, S.; Wegener, T. Harpagophyti radix (Teufelskrallenwurzel). Phytotherapie 2002, 4, 28-33.

419. Chrubasik, S.; Conradt, C. Audiatur et al.tera pars: Teufelskralle in der Diskussion. Z. Phytother. 2002, $23,84-86$.

420. Chrubasik, S.; Conradt, C.; Black, A. The quality of clinical trials with Harpagophytum procumbens. Phytomedicine 2003, 10, 613-623. [CrossRef]

421. Chrubasik, S.; Conradt, C.; Roufogalis, B.D. Effectiveness of Harpagophytum extracts and clinical efficacy. Phytother. Res. 2004, 18, 187-189. [CrossRef] [PubMed]

422. Chrubasik, S.; Eisenberg, E. Treatment of rheumatic pain with kampo medicine in Europe. Part 1. Harpagophytum procumbens. Pain Clin. 1999, 11, 171-178.

423. Chrubasik, S.; Pollak, S. Teufelskrallenwurzelextrakt bei schmerzhafter Arthrose und Rückenschmerzen. Z. Phytother. 2002, 23, 210-215.

424. Chrubasik, S.; Shvartzman, P. Rheumatic pain treatment with devil's claw (Harpagophyti radix). 1999, 1/99. Available online: http:/ / www.iaam.nl/coherence/msaima/199-3.html (accessed on 14 April 2021).

425. Denner, S.S. A review of the efficacy and safety of devil's claw for pain associated with degenerative musculoskeletal diseases, rheumatoid, and osteoarthritis. Holist. Nurse Pr. 2007, 21, 203-207. [CrossRef]

426. Gäbler, H. Harpagophytum procumbens. Homöopath. Mon. 1972, 97, 123-126.

427. Gagnier, J.J.; Chrubasik, S.; Manheimer, E. Harpgophytum procumbens for osteoarthritis and low back pain: A systematic review. BMC Complement. Altern. Med. 2004, 4, 13. [CrossRef] [PubMed]

428. Gagnier, J.J.; Chrubasik, S.; Manheimer, E. Correction: Harpagophytum procumbens for osteoarthritis and low back pain: A systematic review. BMC Complement. Altern. Med. 2005, 5, 1. [CrossRef]

429. Grant, L.; McBean, D.E.; Fyfe, L.; Warnock, A.M. A review of the biological and potential therapeutic actions of Harpagophytum procumbens. Phytother. Res. 2007, 21, 199-209. [CrossRef]

430. Loew, D. Harpagophytum procumbens DC. Eine Übersicht zur Pharmakologie und WIrksamkeit. Erfahrungsheilkunde 1995, 2, 74-79.

431. Savustyanenko, A.V. Эффективность экстракта мартинии душистой(сустамар) приостеоартритах, поясничной боли и фибромиалгии: Обзор исследований. Pain Jt. Spine 2014, 3, 45-53. [CrossRef]

432. Tarleton, A. Devil's claw for low back pain. Herbalgram 1997, 41, 20.

433. Wegener, T. Degenerative Erkrankungen des Bewegungsapparates-Übersicht zu aktuellen klinischen Studien mit Extrakten aus der Teufelskralle (Harpagophyti radix). Wien. Med. Wochenschr. 2002, 152, 389-392. [CrossRef]

434. Ameye, L.G.; Chee, W.S. Osteoarthritis and nutrition. From nutraceuticals to functional foods: A systematic review of the scientific evidence. Arthritis Res. 2006, 8, R127. [CrossRef]

435. Amling, R. Phytotherapeutika in der Neurologie. Z. Phytother. 1991, 12, 9-14.

436. Arndt, D.; Bobermien, K.; Heyer, H.; Hinken, B. Alternative Therapieoptionen bei Endometriose. Gynäkologe 2007, 40, 553558. [CrossRef]

437. Belova, K.Y.; Nazarova, A.V. Стратегия леченияостеоартрита у мультиморбидных пациентов: баланс эффективности и безопасности при выборе лекарственной терапии. Med. Counc. 2020, 11, 164-176. [CrossRef]

438. Brien, S.; Lewith, G.T.; McGregor, G. Devil's claw (Harpagophytum procumbens) as a treatment for osteoarthritis: A review of efficacy and safety. J. Altern. Complement. Med. 2006, 12, 981-993. [CrossRef]

439. Chrubasik, C.; Black, A.; Muller-Ladner, U.; Chrubasik, S. Impact of herbal medicines on physical impairment. Phytomedicine 2008, 15, 536-539. [CrossRef]

440. Chrubasik, J.E.; Roufogalis, B.D.; Chrubasik, S. Evidence of effectiveness of herbal antiinflammatory drugs in the treatment of painful osteoarthritis and chronic low back pain. Phytother. Res. 2007, 21, 675-683. [CrossRef] [PubMed]

441. Chrubasik, S. Osteoarthritis: Pharmacology and clinical indications of selected botanicals. Pain Clin. 2004, 16, 1-16. [CrossRef]

442. Chrubasik, S.; Neumann, E.; Müller-Ladner, U. Zur antientzündlichen Wirksamkeit von Arzneimitteln aus der Teufelskralle. Z. Phytother. 2009, 30, 222-226. [CrossRef]

443. Chrubasik, S.; Pollak, S. Schmerzbehandlung mit pflanzlichen Antirheumatika. Wien. Med. Wochenschr. 2002, 152, 198-203. [CrossRef]

444. Chrubasik, S.; Wink, M. Traditional herbal therapy for the treatment of rheumatic pain: Preparations from devil's claw and stinging nettle. Pain Dig. 1998, 8, 94-101.

445. Chrubasik, S.; Wink, M. Treatment of osteoarthritic pain with herbal drugs. In Proceedings of the 8th World Congress: The Pain Clinic, Tenerife, Spain, 6-10 May 1998; pp. 507-514.

446. Chrubasik-Hausmann, S. Phytotherapie bei Arthrose. Erfahrungsheilkunde 2015, 64, 22-27. [CrossRef] 
447. Corciova, A.; Matei, D.; Ivanescu, B. Medicinal herbs as possible sources of anti-inflammatory products. Balneo Res. J. 2017, 8, 231-241. [CrossRef]

448. Corp, N.; Pendry, B. The role of Western herbal medicine in the treatment of gout. J. Herb. Med. 2013, 3, 157-170. [CrossRef]

449. Darshan, S.; Doreswamy, R. Patented antiinflammatory plant drug development from traditional medicine. Phytother. Res. 2004, 18, 343-357. [CrossRef] [PubMed]

450. Del Grossi Moura, M.; Lopes, L.C.; Biavatti, M.W.; Kennedy, S.A.; de Oliveira, E.S.M.C.; Silva, M.T.; de Cassia Bergamaschi, C. Oral herbal medicines marketed in Brazil for the treatment of osteoarthritis: A systematic review and meta-analysis. Phytother. Res. 2017, 31, 1676-1685. [CrossRef]

451. Deyo, R.A. Conservative therapy for low back pain. Distinguishing useful from useless therapy. JAMA 1983, $250,1057-1062$. [CrossRef] [PubMed]

452. Di Lorenzo, C.; dell'Agli, M.; Badea, M.; Dima, L.; Colombo, E.; Sangiovanni, E.; Restani, P.; Bosisio, E. Plant food supplements with anti-inflammatory properties: A systematic review (II). Crit. Rev. Food Sci. Nutr. 2013, 53, 507-516. [CrossRef]

453. Dragos, D.; Gilca, M.; Gaman, L.; Vlad, A.; Iosif, L.; Stoian, I.; Lupescu, O. Phytomedicine in joint disorders. Nutrients 2017, 9, 70. [CrossRef]

454. Ernst, E.; Chrubasik, S. Phyto-anti-inflammatories. A systematic review of randomized, placebo-controlled, double-blind trials. Rheum. Dis. Clin. N. Am. 2000, 26, 13-27. [CrossRef]

455. Gagnier, J.J. Evidence based review of natural health products for non-specific low back pain. Open Pain J. 2010, 3. [CrossRef]

456. Gagnier, J.J.; van Tulder, M.; Berman, B.; Bombardier, C. Herbal medicine for low back pain. Cochrane Database Syst. Rev. 2006, CD004504. [CrossRef]

457. Gagnier, J.J.; van Tulder, M.W.; Berman, B.; Bombardier, C. Herbal medicine for low back pain: A Cochrane review. Spine 2007, 32, 82-92. [CrossRef] [PubMed]

458. Gregory, P.J.; Sperry, M.; Wilson, A.F. Dietary supplements for osteoarthritis. Am. Fam. Physician 2008, 77, 177-184. [PubMed]

459. Halliwell, B. Drug antioxidant effects. A basis for drug selection? Drugs 1991, 42, 569-605. [CrossRef]

460. Long, L.; Soeken, K.; Ernst, E. Herbal medicines for the treatment of osteoarthritis: A systematic review. Rheumatology 2001, 40, 779-793. [CrossRef]

461. Lopez, H.L. Nutritional interventions to prevent and treat osteoarthritis. Part II: Focus on micronutrients and supportive nutraceuticals. PMR 2012, 4, S155-S168. [CrossRef]

462. Marlowe, D. Complementary and alternative medicine treatments for low back pain. Prim. Care 2012, 39, 533-546. [CrossRef]

463. Oltean, H.; Robbins, C.; van Tulder, M.W.; Berman, B.M.; Bombardier, C.; Gagnier, J.J. Herbal medicine for low-back pain. Cochrane Database Syst. Rev. 2014, CD004504. [CrossRef] [PubMed]

464. Park, K.S. A systematic review on anti-inflammatory activity of harpagoside. J. Biochem. Mol. Biol. Res. 2016, 2, 166-169. [CrossRef]

465. Roth-Utzschneider, S. Erfolgreiche Rheumatherapie mit Teufelskralle und Weidenrinde. Ärztez. Nat. 2006, 47, 488.

466. Rubinstein, S.M.; van Middelkoop, M.; Kuijpers, T.; Ostelo, R.; Verhagen, A.P.; de Boer, M.R.; Koes, B.W.; van Tulder, M.W. A systematic review on the effectiveness of complementary and alternative medicine for chronic non-specific low-back pain. Eur. Spine J. 2010, 19, 1213-1228. [CrossRef] [PubMed]

467. Sanders, M.; Grundmann, O. The use of glucosamine, devil's claw (Harpagophytum procumbens), and acupuncture as complementary and alternative treatments for osteoarthritis. Altern. Med. Rev. 2011, 16, 228.

468. Schmidt, S. Phytotherapie beim rheumatischen Formenkreis. Heilkunst 1973, 86, 18-20.

469. Setty, A.R.; Sigal, L.H. Herbal medications commonly used in the practice of rheumatology: Mechanisms of action, efficacy, and side effects. Semin. Arthritis Rheum. 2005, 34, 773-784. [CrossRef]

470. Soeken, K.L. Selected CAM therapies for arthritis-related pain: The evidence from systematic reviews. Clin. J. Pain. 2004, 20, 13-18. [CrossRef]

471. Spelman, K.; Burns, J.; Nichols, D.; Winters, N.; Ottersberg, S.; Tenborg, M. Modulation of cytokine expression by traditional medicines: A review of herbal immunomodulators. Altern. Med. Rev. 2006, 11, 128-150.

472. Teut, M.; Warning, A. Knochenmetastasen bei Mamma-Karzinom. Komplementmed. 2006, 13, 46-48. [CrossRef]

473. Viljoen, A.; Mncwangi, N.; Vermaak, I. Anti-inflammatory iridoids of botanical origin. Curr. Med. Chem. 2012, 19, 21042127. [CrossRef]

474. Zimmermann, W. Pflanzliche Bitterstoffe in der Gastroenterologie. ZFA 1976, 54, 1178-1184.

475. Zimmermann, W. Interne Phytotherapie der rheumatischen und arthrotischen Erkrankungen. Herba Pol. 1979, 4, $333-342$.

476. Zimmermann, W. Bitterstoffe. Kassenarzt 1985, 8, 44, 47-48.

477. Zimmermann, W. Der obere Dünndarm. Eine Phytotherapiestudie. Therapiewoche 1985, 35, $1592-1602$.

478. Gagnier, J.J.; Oltean, H.; van Tulder, M.W.; Berman, B.M.; Bombardier, C.; Robbins, C.B. Herbal medicine for low back pain: A cochrane review. Spine 2016, 41, 116-133. [CrossRef] [PubMed]

479. Anonymous. Teufelskralle zur Therapie der Osteoarthritis. Dtsch. Apoth. Ztg. 2001, 141, 1-2.

480. Baillard, O. Intérêt de l'Harpagophytum procumbens dans les Affections Rhumatismales. Ph.D. Thesis, Université François Rabelais, Tours, France, 2009; p. 86.

481. Beer, A.M. Teufelskralle und Gymnastik für Knie und Schulter. MMW Med. 2014, 156, 24.

482. Chrubasik, S. Efficacy and safety of Harpagophytum and Salix extract preparations. In Des Sources du Savoir aux Médicaments du Futur; Fleurentin, J., Pelt, J., Mazars, G., Eds.; IRD Éditions: Marseille, France, 2002; ISBN 2709915049. 
483. Chrubasik, S. Weidenrinde und Teufelskralle bei chronischen Gelenk- und Rückenschmerzen: Evidenzlage favorisiert pflanzliche Präparate. Schweiz. Z. Ganzheitsmed. Swiss J. Integr. Med. 2004, 16, 355-359.

484. Chrubasik, S. Zur Evidenz der Wirksamkeit Pflanzlicher Entzündungshemmer bei Arthrose und Rückenschmerzen. Ph.D. Thesis, Klinikum der Albert-Ludwigs-Universität, Freiburg im Breisgau, Germany, 2013; p. 47.

485. Schmidt, S. Therapeutische Wirkung der Teufelskralle. ZFA 1983, 56, 1442.

486. Vanhaelen, M. L'activite de Harpagophytum procumbens et de Glycyrrhiza glabra; Toxicite de drogues contenant des alcaloides derives de la pyrrolizidine. Farm. Tijdschr. Belg. 1986, 63, 31-43.

487. Wenzel, P.; Wegener, T. Teufelskralle-ein pflanzliches Antirheumatikum. Dtsch. Apoth. Ztg. 1995, 135, $15-27$.

488. Zimmermann, W. Harpagophyturn Ampullen D2 der Fa. Hagen. Klinisches Gutachten; Schriftliche Mitteilung an Fa. Hagen: Freilassing, Germany, 1972.

489. Belaiche, P. Un cas de chemosis grave traité avec succès par le nébulisat aqueux de radix Harpagophytum procumbens. Phytotherapy 1983, 5, 15-16.

490. Belaiche, P. Action du nebulisat aqueux de radix Harpagophytum procumbens dans 17 cas de fièvre familiale méditerranéenne (maladie périodique). Phytotherapy 1983, 8, 5-11.

491. Wilson, K.S. Regression of follicular lymphoma with Devil's claw: Coincidence or causation? Curr. Oncol. 2009, 16, 67-70. [CrossRef] [PubMed]

492. Beham, A. Die Anwendung der Harpagophytum-Wurzel bei rheumatischen Erkrankungen. Allg. Homöopath. Ztg. 1971, 216, 204-208. [CrossRef]

493. Brantner, F. Praktische Phytotherapie. Phys. Med. Rehabil. 1972, 13, 87-88.

494. Zimmermann, W. Erfahrungen mit Harpagophytum. Phys. Med. Rehabil. 1977, 18, 317-319.

495. Vanhaelen, M. Mise a jour des recherches relatives a harpagophvtum procumbens. Phytotherapy 1985, 16, 19-24.

496. Stübler, M. Die Behandlung chronischer Gelenkerkrankungen mit Harpagophytum. Allg. Homöopath. Ztg. 1987, 232, 6062. [CrossRef]

497. Stübler, M. Harpagophytum procumbens in rheumatoid arthritis. Br. Homoeopath. J. 1987, 76, 161. [CrossRef]

498. Müller, B.; Deitelhoff, P.; Petrowicz, O. Harpagophytum procumbens ist effizient bei degenerativen Erkrankungen des Bewegungsapparates. Nat. Med. 2000, 21-29.

499. Thanner, J.; Kohlmann, T.; Kunzel, O.; Chrubasik, S. Retrospective evaluation of biopsychosocial determinants and treatment response in patients receiving devil's claw extract (doloteffin). Phytother. Res. 2009, 23, 742-744. [CrossRef] [PubMed]

500. Hoppe, H. Einfluß der Droge Harpagophytum procumbens DC (auch Teufelskralle genannt) auf Diabetes mellitus mit Fettstoffwechselstörungen. Erfahrungsheilkunde 1974, 7, 230-233.

501. Grahame, R.; Robinson, B.V. Devils's claw (Harpagophytum procumbens): Pharmacological and clinical studies. Ann. Rheum. Dis. 1981, 40, 632. [CrossRef]

502. Belaiche, P. Etude clinique de 630 cas d'artrose traites par le nebulisat aqueux d'Harpagophytum procumbens (Radix). Phytotherapy 1982, 1, 22-28.

503. Kröner, W. Wirksamkeit der Homöopathischen Arzneimittel "Formica rufa" und "Harpagophytum procumbens" bei der Gonarthrose: Eine Kontrollierte Studie bei Niedergelassenen Ärzten. Ph.D. Thesis, Universität Ulm, Ulm, Germany, $1991 ;$ p. 82.

504. Moussard, C.; Alber, D.; Toubin, M.M.; Thevenon, N.; Henry, J.C. A drug used in traditional medicine, Harpagophytum procumbens: No evidence for NSAID-like effect on whole blood eicosanoid production in human. Prostaglandins Leukot. Essent. Fat. Acids 1992, 46, 283-286. [CrossRef]

505. Chrubasik, S.; Schmidt, A.; Junck, H.; Pfisterer, M. Wirksamkeit und Wirtschaftlichkeit von Teufelskrallenwurzelextrakt bei Rückenschmerzen: Erste Ergebnisse einer therapeutischen Kohortenstudie. Complement. Med. Res. 1997, 4, 332-336. [CrossRef]

506. Pinget, M.; Lecomte, A. Die Wirkung der "Harpagophytum Arkocaps" bei degenerativem Rheuma. Naturheilpraxis 1997, 50, 267-269.

507. Schwarz, G.; Hämmerle, H.D.; Benvenuti, E. Teufelskralle hilft sanft bei Rückenschmerzen. Allgemeinarzt 1999, $12,1036$.

508. Szczepański, L.; Chudzik, D.; Mazurek, M.; Soroka, P. Badania skuteczności i tolerancji preparatu Pagosid (wyciag z korzenia Harpagophytum procumbens) w leczeniu reumatoidalnego zapalenia stawów i choroby zwyrodnieniowej stawów. Reumatologia 2000, 38, 67-73.

509. Usbeck, C. Teufelskralle: Behandlung chronischer Schmerzen. Arzneim. Forum 2000, 3, $23-25$.

510. Engel, S. Rivoltan (Li 174) zur Behandlung von Patienten mit degenerativen Erkrankungen des Bewegungsapparates. Dtsch. Apoth. Ztg. 2000, 140, 1369.

511. Laudahn, D.; Walper, A. Efficacy and tolerance of Harpagophytum extract LI 174 in patients with chronic non-radicular back pain. Phytother. Res. 2001, 15, 621-624. [CrossRef]

512. Richter, T. Teufelskrallenextrakt bei chronischen Rückenschmerzen. Dtsch. Apoth. Ztg. 2002, 142, 131-133.

513. Ullmann, M. Therapie chronischer Rückenschmerzen: Teufelskrallen-Extrakt erzielt hochsignifikante Schmerzreduktion. Allgemeinarzt 1999, 11, 1018-1019.

514. Schendel, U.M. Arthrose-therapie: Verträglich geht es auch. Kassenarzt 2001, 29, 36-39.

515. Ribbat, J.M.; Schakau, D. Behandlung chronisch aktivierter Schmerzen am Bewegungsapparat. Nat. Med. 2001, 16, 23-32. 
516. Chrubasik, S.; Thanner, J.; Künzel, O.; Conradt, C.; Black, A.; Pollak, S. Comparison of outcome measures during treatment with the proprietary Harpagophytum extract doloteffin in patients with pain in the lower back, knee or hip. Phytomedicine 2002, 9, 181-194. [CrossRef]

517. Flammersfeld, L.; Weinmann, B. Traitement du rhumatisme par l'extrait sec de racine d'Harpagophytum. Une étude multicentrique en cabinet médical. Phytotherapie 2005, 3, 104-106. [CrossRef]

518. Kloker, B.; Flammersfeld, L. Rheumatherapie mit Teufelskrallenwurzel-Trockenextrakt. Eine multizentrische Praxisstudie. Ärztez. Nat. 2003, 44, 108-111.

519. Wegener, T.; Lüpke, N.P. Treatment of patients with arthrosis of hip or knee with an aqueous extract of devil's claw (Harpagophytum procumbens DC.). Phytother. Res. 2003, 17, 1165-1172. [CrossRef]

520. Wegener, T. Zur Klinischen Wirksamkeit der Südafrikanischen Teufelskrallenwurzel (Harpagophyti radix) bei Patienten mit Cox-und Gonarthrose: Ergebnisse und Bewertung Einer Klinischen Studie der Phase IV. Ph.D. Thesis, Universität Osnabrück, Osnabrück, Germany, 2005; p. 115.

521. Rütten, S.; Kuhn, M. Spezialextrakt aus Teufelskrallenwurzel. Klinische Vergleichsstudie bei Patienten mit unspezifischen Schmerzen im Lumbalbereich. Ärztez. Nat. 2005, 46, 114-116.

522. Schmidt, A.; Berghof, U.; Schmidt, E. Therapie der unspezifischen Lumbalgie mit Teufelskrallenwurzelextrakt-Ergebnisse einer klinischen Studie. Phys. Med. Rehabil. Kurortmed. 2005, 15, 317-321. [CrossRef]

523. Schmidt, A.P.; Schmidt, E.G. Über die Wirksamkeit von Teufelskrallenwurzelextrakt zur Therapie Unspezifischer Lumbaler Rückenschmerzen; Cuvillier Verlag: Göttingen, Germany, 2005; p. 101, ISBN 3865376118.

524. Sohail, M.T.; Chaudhry, M.I.; Usman, M.K.; Mian, T.; Ishaq, M.N. Efficacy and tolerance of atrisin in degenerative and inflammatory joint disorders. Phytother. Res. 2005, 19, 365-368. [CrossRef] [PubMed]

525. Arndt, D.; Bobermien, K.; Heyer, H.; Braun, D.; Köhler, G. Harpagophytum procumbens—Erste Erfahrungen in der Endometriosetherapie. Zent. Gynakol. 2005, 127, A1. [CrossRef]

526. Arndt, D.; Bobermien, K.; Heyer, H.; Köhler, G. Hormonfreie Endometriosetherapie mit Harpagophytum procumbens—Ein neuer Weg in der Endometriosetherapie. Geburtshilfe Frauenheilkd. 2006, 66, PO_E_03_10. [CrossRef]

527. Suter, A.; Whittaker, P.; Dickson, S.; McIntyre, L.; Tan, J. Positive influence of a Harpagophytum procumbens preparation on different rheumatic complaints-Results from clinical trial. Planta Med. 2006, 72, P_200. [CrossRef]

528. Warnock, M.; McBean, D.; Suter, A.; Tan, J.; Whittaker, P. Effectiveness and safety of Devil's claw tablets in patients with general rheumatic disorders. Phytother. Res. 2007, 21, 1228-1233. [CrossRef] [PubMed]

529. Chrubasik, S.; Chrubasik, C.; Kunzel, O.; Black, A. Patient-perceived benefit during one year of treatment with Doloteffin. Phytomedicine 2007, 14, 371-376. [CrossRef] [PubMed]

530. Conrozier, T.; Mathieu, P.; Bonjean, M.; Marc, J.F.; Renevier, J.L.; Balblanc, J.C. A complex of three natural anti-inflammatory agents provides relief of osteoarthritis pain. Altern. Health Med. 2014, 20, 32-37.

531. Vreju, F.A.; Ciurea, P.L.; Rosu, A.; Chisalau, B.A.; Parvanescu, C.D.; Firulescu, S.C.; Turcu Stiolica, A.; Barbulescu, A.L.; Dinescu, S.C.; Dumitrescu, C.I. The effect of glucosamine, chondroitin and Harpagophytum procumbens on femoral hyaline cartilage thickness in patients with knee osteoarthritis-An MRI versus ultrasonography study. J. Mind Med. Sci. 2019, 6, 162-168. [CrossRef]

532. Hu, S.; Belcaro, G.; Cesarone, M.R.; Feragalli, B.; Cotellese, R.; Dugall, M.; Scipione, C.; Scipione, V.; Maione, C.; Maramaldi, G.; et al. A sport cream (Harpago-Boswellia-ginger-escin) for localized neck/shoulder pain. Minerva Med. 2021, 112, 255-260. [CrossRef] [PubMed]

533. Schrüffler, H. Ein Fortschritt in der nichtsteroidalen antirheumatischen Therapie. Med. Publ. 1980, 1, 1-8.

534. Guyader, M. Les Plantes Anti-Rhumatismales. Etude Historique et Pharmacologique. Et Etude Clinique du Nebulisat d'Harpagophytum procumbens DC chez 50 Patients Arthrosioues Suivis en Service Hospitalier. Ph.D. Thesis, Université Pierre et Marie Curie, Paris, France, 1984; p. 151.

535. Pinget, M.; Lecomte, A. Etude des effets de l'Harpagophytum en rhumatologie dégénérative. 37² Le Mag. 1990, $10,23-25$.

536. Lecomte, A.; Costa, J.P. Harpagophytum dans l'arthrose: Etude en double insu contre placebo. 37² Le Mag. 1992, 15, 27-30.

537. Chrubasik, S.; Zimpfer, C.; Schütt, U.; Ziegler, R. Effectiveness of Harpagophytum procumbens in treatment of acute low back pain. Phytomedicine 1996, 3, 1-10. [CrossRef]

538. Chrubasik, S.; Pollak, S.; Black, A. Effectiveness of devil's claw for osteoarthritis. Rheumatology 2002, 41, 1332-1333, author reply 1333. [CrossRef]

539. Chrubasik, S.; Fiebich, B.; Black, A.; Pollak, S. Treating low back pain with an extract of Harpagophytum that inhibits cytokine release. Eur. J. Anaesthesiol. 2002, 19, 209. [CrossRef]

540. Chrubasik, S.; Zimpfer, C.; Schütt, U.; Ziegler, R. The arhus low back rating scale: A useful tool to evaluate the success of low back pain treatment. Reg. Anesth. J. Neural Blockade Obstet. Surg. Pain Control 1997, 22, 100.

541. Schmelz, H.; Hämmerle, H.D.; Springorum, H.W. Analgetische Wirkung eines Teufelskrappenwurzel-Extraktes bei verschiedenen chronisch-degenrativen Gelenkerkrankungen. In Rheumatherapie mit Phytopharmaka; Chrubasik, S., Wink, M., Eds.; Hippokrates: Stuttgart, Germany, 1997; pp. 86-89.

542. Chrubasik, S.; Junck, H.; Breitschwerdt, H.; Conradt, C.; Zappe, H. Effectiveness of Harpagophytum extract WS 1531 in the treatment of exacerbation of low back pain: A randomized, placebo-controlled, double-blind study. Eur. J. Anaesthesiol. 1999, 16, 118-129. [CrossRef] [PubMed] 
543. Chantre, P.; Cappelaere, A.; Leblan, D.; Guedon, D.; Vandermander, J.; Fournie, B. Efficacy and tolerance of Harpagophytum procumbens versus diacerhein in treatment of osteoarthritis. Phytomedicine 2000, 7, 177-183. [CrossRef]

544. Leblan, D.; Chantre, P.; Fournié, B. L'harpagophyton dans le traitement de la gonarthrose et de la coxarthrose. Résultats à quatre mois d'une étude prospective multicentrique, contrôlée en double aveugle, versus diacerhéine. Rev. Rhum. 2000, 67, 634-640. [CrossRef]

545. Göbel, H.; Heinze, A.; Ingwersen, M.; Niederberger, U.; Gerber, D. Wirkmechanismus von Harpagophytum-procumbens-Extrakt L1 174 bei der Behandlung von unspezifischen Rückenschmerzen. In Phytopharmaka VI, Forschung und klinische Anwendung; Rietbrock, N., Ed.; Steinkopff: Darmstadt, Germany, 2000; pp. 99-115.

546. Göbel, H.; Heinze, A.; Ingwersen, M.; Niederberger, U.; Gerber, D. Harpagophytum-Extrakt LI 174 (Teufelskralle) bei der Behandlung unspezifischer Ruckenschmerzen-Effekte auf die sensible, motorische und vaskulare Muskelreagibilitat. Schmerz 2001, 15, 10-18. [CrossRef]

547. Frerick, H.; Biller, A.; Schmidt, U. Stufenschema bei Coxarthrose. Kassenarzt 2001, 5, 34-41.

548. Biller, A. Ergebnisse zweier randomisierter kontrollierter Studien und einer Anwendungsbeobachtung mit Teufelskrallenextrakt. In Phytopharmaka VII, Forschung und klinische Anwendung; Schulz, V., Rietbrock, N., Roots, I., Loew, D., Eds.; Steinkopff: Darmstadt, Germany, 2002; pp. 81-92, ISBN 978-3-642-57528-0.

549. Chrubasik, S.; Model, A.; Black, A.; Pollak, S. A randomized double-blind pilot study comparing Doloteffin and Vioxx in the treatment of low back pain. Rheumatology 2003, 42, 141-148. [CrossRef]

550. Chrubasik, S.; Künzel, O.; Thanner, J.; Conradt, C.; Black, A. A 1-year follow-up after a pilot study with Doloteffin for low back pain. Phytomedicine 2005, 12,1-9. [CrossRef]

551. Chrubasik, S.; Künzel, O.; Thanner, J.; Conradt, C. A short-term follow-up after a randomised double-blind pilot study comparing Doloteffin ${ }^{\circledR}$ vs. rofecoxib for low back pain. Focus Altern. Complement. Ther. 2003, 8, 133. [CrossRef]

552. Ullmann, M.; Model, A.; Chrubasik, S. Treatment of acute low back pain with the Harpagophytum extract Doloteffin and the COX-2 inhibitor VIOXX: A pilot study. In Proceedings of the 3rd International Congress on Phytomedicine, Munich, Germany, 11-13 October 2000.

553. Lienert, A.; Rütten, S.; Kuhn, M.; Wartenberg-Demand, A. A randomised, active-controlled, mono-centric study of the herbal drug, Devil's claw (Harpagophytum procumbens) (ALLYA ${ }^{\circledR}$ tablets), Voltaren ${ }^{\circledR}$ and Viox ${ }^{\circledR}$ indicates equal efficacy in the treatment of patients with unspecific lumbar pain. 54. Jahrestag. Norddtsch. Orthopädenver. Eingetrag. Ver. Hambg. 2005, 16, Doc05novEP26.

554. Lienert, A.; Rütten, S.; Kuhn, M.; Wartenberg-Demand, A. Efecto analgésico de un extracto estandarizado de harpagofito. Rev. Fitoter. 2007, 7, 15-20.

555. Pillay, D. A Double-Blinded, Placebo Controlled Clinicaltrial Evaluating the Efficacy of the Harpago and Celery Seed Cream in Mild to Moderate Degenerative Joint Disease of the Knee. Ph.D. Thesis, Durban Institute of Technology, Durban, South Africa, 2006; p. 138.

556. Anvari, H.M.P.; Panahi, J.R.; Ansari, M.; Iraji, M.; Ghorbanian, N.; Dehghani, A. The effect of single dose of Harpagophytum capsule (teltonal) on post tracheal intubation sore throat after general anesthesia. Arch. Anesthesiol. Crit. Care 2016, 2, $239-242$.

557. Moré, M.; Grünwald, J.; Pohl, U.; Uebelhack, R. A Rosa canina-Urtica dioica-Harpagophytum procumbens/zeyheri combination significantly reduces gonarthritis symptoms in a randomized, placebo-controlled double-blind study. Planta Med. 2017, 83, 1384-1391. [CrossRef] [PubMed]

558. Anonymous. Devil's claw root: Ulcers and gastrointestinal bleeding? Prescrire Int. 2013, 22, 296.

559. Gallo, E.; Lucenteforte, E.; Firenzuoli, F.; Menniti-Ippolito, F.; Maggini, V.; Pugi, A.; Mascherini, V.; Gori, L.; Mugelli, A.; Vannacci, A. Herbalists' perception of risks involving commonly sold medicinal plants in Italy. Complement. Med. 2014, $22,81-86$. [CrossRef] [PubMed]

560. Vlachojannis, J.; Roufogalis, B.D.; Chrubasik, S. Systematic review on the safety of Harpagophytum preparations for osteoarthritic and low back pain. Phytother. Res. 2008, 22, 149-152. [CrossRef]

561. Posadzki, P.; Watson, L.K.; Ernst, E. Adverse effects of herbal medicines: An overview of systematic reviews. Clin. Med. 2013, 13, 7-12. [CrossRef]

562. Biazi, B.I.; D'Epiro, G.F.; Zanetti, T.A.; de Oliveira, M.T.; Ribeiro, L.R.; Mantovani, M.S. Risk assessment via metabolism and cell growth inhibition in a HepG2/C3A cell line upon treatment with arpadol and its active component harpagoside. Phytother. Res. 2017, 31, 387-394. [CrossRef]

563. Cordier, W.; Steenkamp, V. Drug interactions in African herbal remedies. Drug Metab. Drug Interact. 2011, 26, 53-63. [CrossRef]

564. Shaw, D.; Leon, C.; Kolev, S.; Murray, V. Traditional remedies and food supplements. A 5-year toxicological study (1991-1995). Drug Saf. 1997, 17, 342-356. [CrossRef] [PubMed]

565. Argento, A.; Tiraferri, E.; Marzaloni, M. Anticoagulanti orali e piante medicinali. Una interazione emergente. Ann. Ital. Med. Int. 2000, 15, 139-143.

566. Heck, A.M.; DeWitt, B.A.; Lukes, A.L. Potential interactions between alternative therapies and warfarin. Am. J. Health Syst. Pharm. 2000, 57, 1221-1227. [CrossRef] [PubMed]

567. Ramsay, N.A.; Kenny, M.W.; Davies, G.; Patel, J.P. Complimentary and alternative medicine use among patients starting warfarin. Br. J. Haematol. 2005, 130, 777-780. [CrossRef]

568. Izzo, A.A.; Di Carlo, G.; Borrelli, F.; Ernst, E. Cardiovascular pharmacotherapy and herbal medicines: The risk of drug interaction. Int. J. Cardiol. 2005, 98, 1-14. [CrossRef] 
569. Carvalho, R.R.; Donadel, C.D.; Cortez, A.F.; Valviesse, V.R.; Vianna, P.F.; Correa, B.B. Syndrome of inappropriate antidiuretic hormone secretion induced by the phytotherapy Harpagophytum procumbers: Case report. J. Bras. Nefrol. 2017, 39, 79-81. [CrossRef] [PubMed]

570. Cuspidi, C.; Sala, C.; Tadic, M.; Grassi, G.; Mancia, G. Systemic Hypertension Induced by Harpagophytum procumbens (Devil's claw): A case report. J. Clin. Hypertens. 2015, 17, 908-910. [CrossRef] [PubMed]

571. Douros, A.; Bronder, E.; Andersohn, F.; Klimpel, A.; Thomae, M.; Ockenga, J.; Kreutz, R.; Garbe, E. Drug-induced acute pancreatitis: Results from the hospital-based Berlin case-control surveillance study of 102 cases. Aliment. Pharmacol. Ther. 2013, 38, 825-834. [CrossRef]

572. Altmeyer, N.; Garnier, R.; Rosenberg, N.; Geerolf, A.M.; Ghaem, A. Conjonctivite, rhinite et asthme rythmes par l'exposition professionnelle a l'Harpagophytum. Arch. Mal. Prof. Méd. Trav. Sécurité Soc. 1991, 53, 289-291.

573. Rahman, H.; Kim, M.; Leung, G.; Green, J.A.; Katz, S. Drug-herb interactions in the elderly patient with IBD: A growing concern. Curr. Treat. Options Gastroenterol. 2017, 15, 618-636. [CrossRef]

574. Colas, C. Développement de Méthodes Physico-Chimiques pour le Contrôle de la Médicationpar l'Harpagophytum et l'Eleutherococcus, Principes Actifs Utilisés en Phytothérapie Équine. Ph.D. Thesis, École Polytechnique, Université Paris-Saclay, Paris, France, 2006; p. 313.

575. Torfs, S.; Delesalle, C.; Vanschandevijl, K.; de Clercq, D.; Van Loon, G.; Nollet, H.; Deprez, P. Anti-inflammatory phytotherapeutics: A valuable alternative to NSAID treatment in horses. Vlaams Diergeneeskd. Tijdschr. 2008, 78, 161-170.

576. Montavon, S. Efficacité d'une préparation phytothérapique à base d'Harpagophytum procumbens dans les cas d'éparvin chez le cheval adulte. Prat. Vét. Equine 1994, 26, 49-53.

577. Axmann, S. Studien zur Pharmakokinetik und zur Klinischen Wirksamkeit von Harpagosid aus Einem Extrakt der Teufelskralle ("Harpagophytum procumbens" DC ex Meisn.) bei Pferden. Ph.D. Thesis, Veterinärmedizinische Universität, Wien, Austria, 2019 ; p. 106.

578. Axmann, S.; Hummel, K.; Nöbauer, K.; Razzazi-Fazeli, E.; Zitterl-Eglseer, K. Pharmacokinetics of harpagoside in horses after intragastric administration of a Devil's claw (Harpagophytum procumbens) extract. J. Vet. Pharmacol. Ther. 2019, $42,37-44$. [CrossRef] [PubMed]

579. Moreau, M.; Lussier, B.; Pelletier, J.P.; Martel-Pelletier, J.; Bedard, C.; Gauvin, D.; Troncy, E. A medicinal herb-based natural health product improves the condition of a canine natural osteoarthritis model: A randomized placebo-controlled trial. Res. Vet. Sci. 2014, 97, 574-581. [CrossRef] 